\title{
Ni-Catalyzed Reductive Cyanation of Aryl Halides and Phenol Derivatives via Transnitrilation
}

\author{
L. Reginald Mills, Joshua Graham, ${ }^{*}$ Purvish Patel, ${ }^{\star}$ and Sophie A. L. Rousseaux* \\ Davenport Research Laboratories, Department of Chemistry, University of Toronto \\ 80 St. George St., Toronto, ON, M5S 3H6 \\ \$These authors contributed equally; *Corresponding author: sophie.rousseaux@utoronto.ca
}

\section{Supporting Information 1: Experimental Data}

\section{Table of contents}

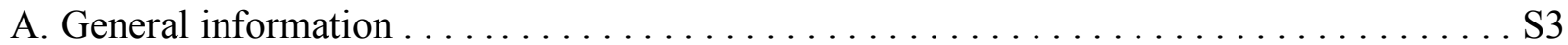

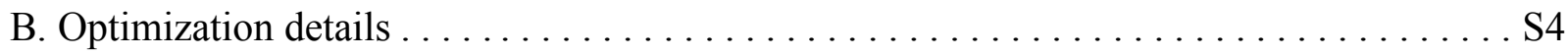

Table S1. Optimization of the reductive cyanation reaction for aryl bromides . . . . . S5

Table S2. Evaluation of transfer reagents and reductants . . . . . . . . . . S6

Table S3. Evaluation of temperature and solvents . . . . . . . . . . S6

Table S4. Optimization of the reductive cyanation reaction for aryl tosylates . . . . S7

Table S5. Evaluation of the reductive cyanation reaction for aryl chlorides . . . . . S8

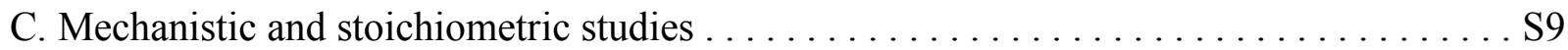

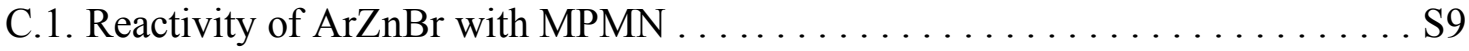

Scheme S1. Reactivity of ArZnBr with MPMN . . . . . . . . . . . . . . S9



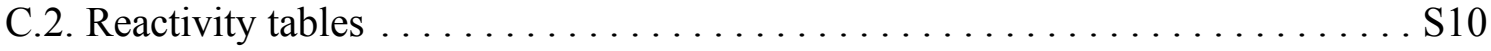

Table S7. Reactivity of post-oxidative addition complex 4 with MPMN . . . . . . . S10

Table S8. Transnitrilation in the presence of chloride additives . . . . . . . . S10

Equation $\mathrm{S} 1$. Reaction of MPMN with $\mathrm{NiBr}_{2}($ bpy $) \bullet \mathrm{xH}_{2} \mathrm{O}$ and $\mathrm{Zn}(0)$ dust $\ldots . . . \mathrm{S} 11$

C.3. Testing reaction mixture for cyanide using $p$-benzoquinone $\ldots \ldots \ldots \ldots \ldots$ S12

Figure $\mathrm{S} 1 . p$-Benzoquinone test fluorescence data $\ldots \ldots \ldots \ldots \ldots \ldots \ldots \ldots \ldots$

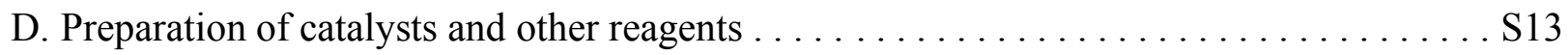

Figure S2. ORTEP structure of complex $4 \ldots \ldots \ldots \ldots \ldots \ldots \ldots \ldots \ldots \ldots \ldots \ldots$ 


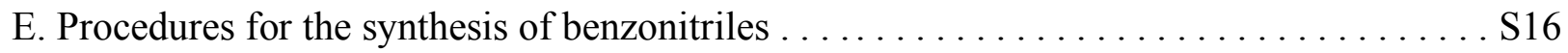

Figure S3. Representative unsuccessful substrates . . . . . . . . . . . . . S17

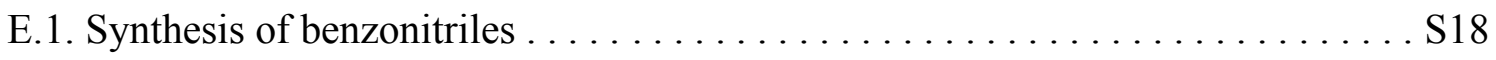

Table S10. Supplemental scope table ........................... S18

E.2. Synthesis of biologically relevant benzonitriles . . . . . . . . . . . . S31

E.3. Synthesis of benzonitriles from alternate electrophiles using $\mathrm{NaBr} \ldots \ldots \ldots \mathrm{S} 32$



F.1. Synthesis of 2-methyl-2-phenylmalononitrile (MPMN) ............. S35

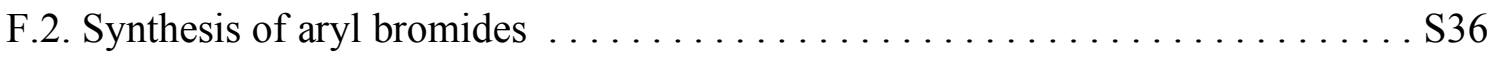

F.3. Synthesis of starting materials for biologically relevant benzonitriles . . . . . . . S44

F.4. Synthesis of alternate aryl electrophiles . ..................... S47

G. Preparation of GC-MS standards . . . . . . . . . . . . . . . . . . . . . . S 49

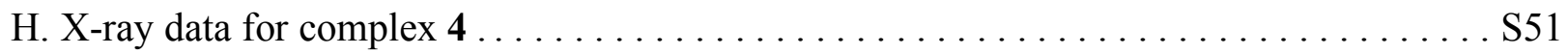






\section{A. General information}

Unless otherwise noted, all reactions were set up on the benchtop and run under an atmosphere of $\mathrm{Ar}$ or $\mathrm{N}_{2}$ using flame-dried glassware and anhydrous solvents. $\mathrm{CH}_{2} \mathrm{Cl}_{2}, \mathrm{Et}_{2} \mathrm{O}, \mathrm{MeCN}, \mathrm{PhMe}$, and THF were purchased as HPLC-grade (inhibitor-free) from Caledon or Sigma-Aldrich, and were dried using a PureSolv MD 5 solvent purification system and used without further manipulation. DMA and DMF were purchased from Acros as Extra Dry over molecular sieves and were used as received. For bottles of DMA older than 30 days, DMA was sparged with Ar for $15 \mathrm{~min}$ before use. DMSO was purchased as reagent-grade and was dried over $3 \AA$ molecular sieves for 3 days. $\mathrm{NiBr}_{2}$ (bpy) $\bullet \mathrm{xH}_{2} \mathrm{O}$ was prepared as anhydrous $\mathrm{NiBr}_{2}$ (bpy) from $\mathrm{NiBr}_{2} \bullet 3 \mathrm{H}_{2} \mathrm{O}$ and bpy $;^{1}$ while not highly hygroscopic, the anhydrous catalyst was stored in a dessicator open to air and was weighed on benchtop and was assumed to have some amount of hydrate. $\mathrm{NiBr}_{2}(\text { bpy })_{2} \cdot 2 \mathrm{H}_{2} \mathrm{O}$ was prepared from $\mathrm{NiBr}_{2} \bullet 3 \mathrm{H}_{2} \mathrm{O}$ and bpy (2 equiv). ${ }^{2} \mathrm{NiBr}_{2}(\text { bpy })_{2}{ }^{3}$ and $\mathrm{NiBr}_{2}(\text { bpy })_{3}{ }^{4}$ were prepared according to known procedures. $\mathrm{Zn}(0)$ dust was activated with $\mathrm{HCl}$ before use. ${ }^{5}$ Dimethylmalononitrile (DMMN) and dibenzylmalononitrile (DBMN) were prepared as previously reported. ${ }^{6} \mathrm{~N}$-Tetrabutylammonium chloride (TBAC), $\mathrm{LiBr}, \mathrm{MgBr}_{2}$, and $\mathrm{ZnBr}_{2}$ were purchased as anhydrous reagents and were stored in a glovebox. $N$-Tetrabutylammonium bromide (TBAB) was purchased as reagent grade and was dried using $\mathrm{P}_{2} \mathrm{O}_{5}$ for $12 \mathrm{~h}$ and was stored in a glovebox. All other commercial reagents and starting materials were used as received. Compounds were purified by flash column chromatography using SiliCycle SilicaFlash P60 silica gel.

GC-MS data was obtained on a Shimadzu GCMS-QP2010 SE; yields represent peak areas calibrated against each compound's response factor relative to $n$-dodecane internal standard. ${ }^{1} \mathrm{H}$ and ${ }^{13} \mathrm{C}$ NMR spectra were recorded on Varian MercuryPlus $400 \mathrm{MHz}$, Agilent DD2 $500 \mathrm{MHz}$, or Bruker AvanceIII $400 \mathrm{MHz}$ spectrometers. TLC samples were run on EMD Millipore TLC Silica gel $60 \mathrm{~F}_{254}$ plates and were visualized by UV or by staining with standard $\mathrm{KMnO}_{4}$, phosphomolybdic acid (PMA), or $p$-anisaldehyde stains. IR spectra were obtained on a PerkinElmer Spectrum 100 instrument equipped with a single-bounce diamond/ZnSe ATR accessory as solids or thin films. Melting points were obtained on a Fisher-Johns Melting Point Apparatus. High-resolution mass spectra (HRMS) were recorded on a JEOL AccuTOF JMS-T1000LV mass spectrometer equipped with a Direct Analysis in Real Time (DART) ion source. Fluorescence spectra were recorded on a Photon Technology International (PTI) QuantaMaster 40-F NA spectrofluorometer using a xenon arc light source and 914 Photomultiplier Detection System; samples were excited using $400 \mathrm{~nm}$ light and emission spectra recorded from 450 to $500 \mathrm{~nm}$ at r.t. $\left(23^{\circ} \mathrm{C}\right)$. 


\section{B. Optimization details}

Caution: Depending on the reductant, ligand, and nickel catalyst used, decyanation of excess MPMN was sometimes observed, implying formation of cyanide salts in situ. As a precaution, all reactions were handled under basic conditions and were disposed of appropriately.

Procedure for Tables S1-S3: To a flame-dried 8-mL culture tube with a stir bar were added $\mathrm{Zn}(0)$ dust (13 $\mathrm{mg}, 0.20 \mathrm{mmol}, 2.0$ equiv), Ni source $(0.010 \mathrm{mmol}, 10 \mathrm{~mol} \%)$, and ligand. The tube was sealed with a size 19 septum and electrical tape, evacuated and backfilled with $\mathrm{N}_{2}(\times 3)$, and DMA $(0.25 \mathrm{~mL})$ was added. The reaction was stirred at r.t. for $2 \mathrm{~min}$. MPMN $(0.25 \mathrm{~mL}$ of a $0.48 \mathrm{M}$ stock solution in DMA, $0.12 \mathrm{mmol}, 1.2$ equiv) was added, followed by 3-bromoanisole (13 $\mu \mathrm{L}, 0.10 \mathrm{mmol}, 1.0$ equiv), and the tube was immediately placed into a pre-heated $80{ }^{\circ} \mathrm{C}$ oil bath. The reaction was stirred for $16 \mathrm{~h}$ at $80^{\circ} \mathrm{C}$. The reaction was cooled to r.t., opened to air, and quenched with sat. aq. $\mathrm{NaHCO}_{3} . n$-Dodecane $(23 \mu \mathrm{L}, 0.10 \mathrm{mmol}, 1.0$ equiv) was added as an internal standard for GC-MS analysis. The reaction was extracted with EtOAc $(\times 2)$ and the organic fractions were combined and filtered over a plug of $\mathrm{MgSO}_{4}$ and Celite. The crude solution was analyzed by GC-MS. 
Table S1. Optimization of the reductive cyanation reaction for aryl bromides

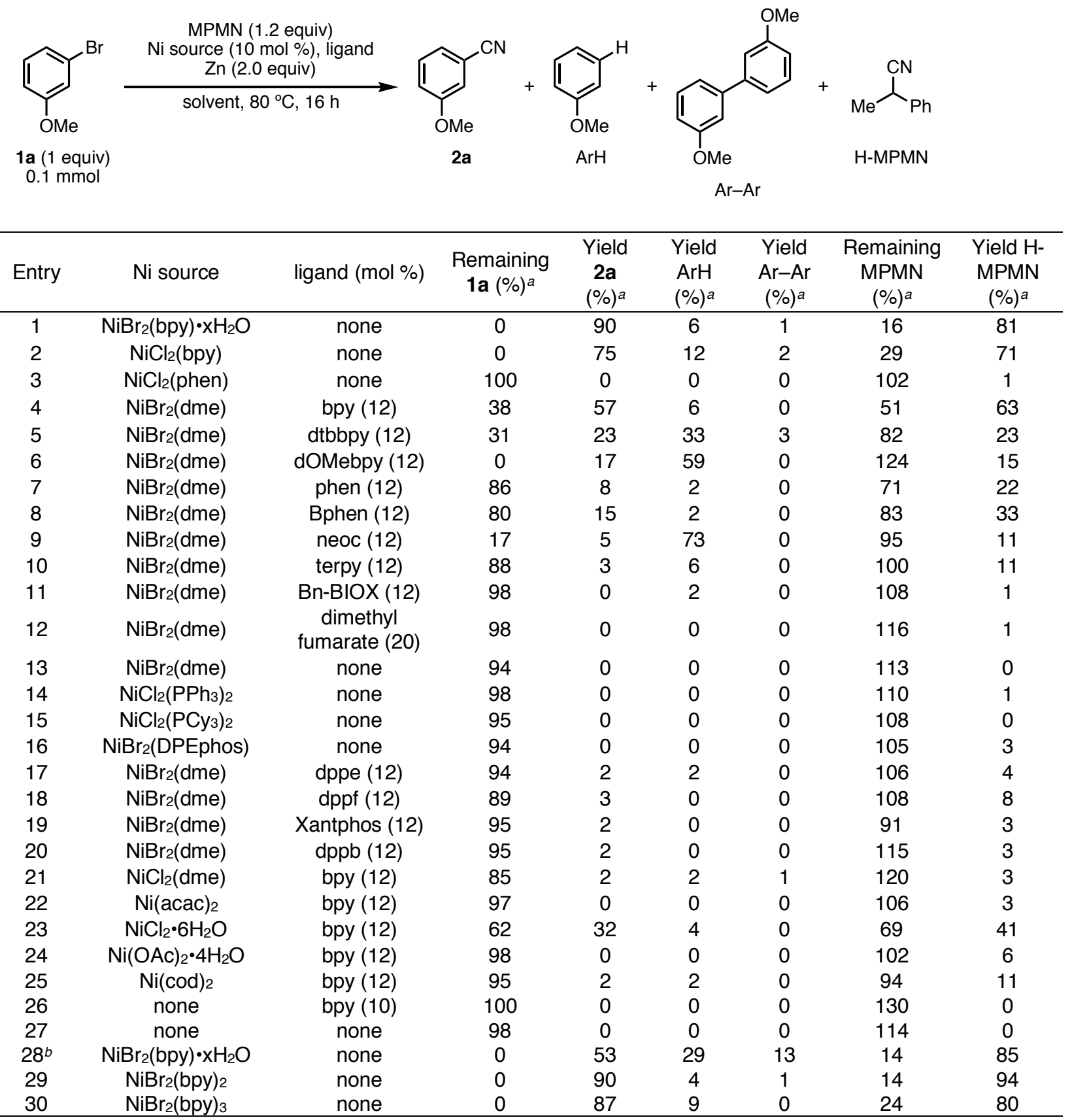

aDetermined by GC-MS using $n$-dodecane as internal standard.

bUsing 3-iodoanisole instead of 3-bromoanisole. 
Table S2. Evaluation of transfer reagents and reductants

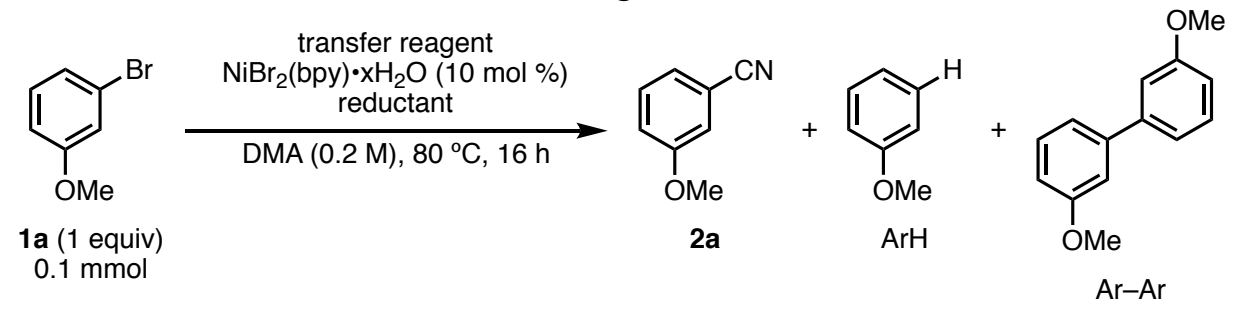

\begin{tabular}{ccccccc}
\hline Entry & $\begin{array}{c}\text { transfer reagent } \\
\text { (equiv) }\end{array}$ & $\begin{array}{c}\text { reductant } \\
\text { (equiv) }\end{array}$ & $\begin{array}{c}\text { Remaining 1a } \\
(\%)\end{array}$ & Yield 2a (\%) & Yield ArH (\%) & $\begin{array}{c}\text { Yield Ar-Ar } \\
(\%)\end{array}$ \\
\hline 1 & Zn (2.0) & MPMN (1.2) & 0 & 90 & 6 & 1 \\
2 & Zn (2.0) & DBMN (1.2) & 0 & 10 & 47 & 17 \\
3 & Zn (2.0) & DMMN (1.2) & 0 & 17 & 39 & 19 \\
4 & Mn (2.0) & MPMN (1.2) & 17 & 77 & 2 & 0 \\
\hline
\end{tabular}

aDetermined by GC-MS using $n$-dodecane as internal standard.



MPMN = 2-methyl-2-phenylmalononitrile; DMMN = dimethylmalononitrile; DBMN = dibenzylmalononitrile.

Table S3. Evaluation of temperature and solvents



\begin{tabular}{ccccccc}
\hline Entry & Solvent (M) & $\begin{array}{c}\text { Temperature } \\
\left({ }^{\circ} \mathrm{C}\right)\end{array}$ & $\begin{array}{c}\text { Remaining 1a } \\
(\%)^{a}\end{array}$ & $\begin{array}{c}\text { Yield 2a (\%) } \\
\text { ( }\end{array}$ & $\begin{array}{c}\text { Yield Ar-Ar }(\%)^{a} \\
(\%)^{a}\end{array}$ \\
\hline 1 & DMA (0.2) & 80 & 0 & 90 & 6 & 1 \\
2 & DMA (0.2) & 50 & 100 & 2 & 0 & 0 \\
3 & DMF (0.2) & 80 & 25 & 65 & 6 & 0 \\
4 & MeCN (0.2) & 80 & 105 & 0 & 0 & 0 \\
5 & PhMe (0.2) & 80 & 102 & 0 & 0 & 0 \\
6 & THF (0.2) & 80 & 97 & 0 & 0 & 0 \\
7 & 1,4-dioxane (0.2) & 80 & 100 & 0 & 0 & 0 \\
8 & DMA (0.2) & 80 & 1 & 87 & 5 & 0 \\
9 & DMA (0.2) & 80 & 100 & 0 & 0 & 0 \\
\hline
\end{tabular}

aDetermined by GC-MS using $n$-dodecane as internal standard.

bDMA was sparged with air for 15 min before use.

cNo precautions; using reagent-grade DMA without flame-dried glassware under air. 
Table S4. Optimization of the reductive cyanation reaction for aryl tosylates

\begin{tabular}{|c|c|c|c|c|c|}
\hline \multirow{4}{*}{ OMe } & \multirow{4}{*}{$\begin{array}{c}\text { MPMN (1 equiv) } \\
\mathrm{NiBr}_{2}(\text { bpy }) \cdot \mathrm{xH}_{2} \mathrm{O}(20 \mathrm{~mol} \%) \\
\text { additive } \\
\mathrm{Zn}(2 \text { equiv }) \\
\text { DMA }(0.2 \mathrm{M}), 80^{\circ} \mathrm{C}, 16 \mathrm{~h}\end{array}$} & \multirow{4}{*}{\multicolumn{3}{|c|}{ ArH }} & \multirow[b]{5}{*}{ Yield Ar-Ar (\%) ${ }^{a}$} \\
\hline & & & & & \\
\hline & & & & & \\
\hline & & & & & \\
\hline Entry & Additive (equiv) & $\begin{array}{l}\text { Remaining } \\
\text { ArOTs }(\%)^{a}\end{array}$ & Yield $\mathbf{2 a}(\%)^{a}$ & Yield ArH (\%) ${ }^{a}$ & \\
\hline 1 & none & 93 & 0 & 0 & 0 \\
\hline 2 & $\mathrm{NaBr}(2)$ & 0 & 82 & 12 & 0 \\
\hline $3^{b}$ & $\mathrm{NaBr}(2)$ & 91 & 5 & 0 & 0 \\
\hline 4 & Nal (2) & 94 & 0 & 0 & 0 \\
\hline 5 & $\mathrm{NaCl}(2)$ & 93 & 0 & 0 & 0 \\
\hline 6 & $\mathrm{LiBr}(2)$ & 0 & 75 & 8 & 1 \\
\hline 7 & TBAC (2) & 6 & 75 & 2 & 0 \\
\hline 8 & $\mathrm{MgBr}_{2}(1)$ & 59 & 32 & 4 & 0 \\
\hline 9 & TBAB (2) & 0 & 78 & 10 & 1 \\
\hline 10 & TBAB (2) & 0 & 78 & 10 & 2 \\
\hline 11 & $\mathrm{NaBr}(1)$ & 48 & 42 & 0 & 0 \\
\hline 12 & $\mathrm{KBr}(2)$ & 97 & 0 & 0 & 0 \\
\hline 13 & $\mathrm{CsBr}(2)$ & 102 & 0 & 0 & 0 \\
\hline 14 & $\mathrm{ZnBr}_{2}(1)$ & 99 & 0 & 0 & 0 \\
\hline 15 & $\mathrm{KI}(2)$ & 98 & 2 & 0 & 0 \\
\hline 16 & TBAI (2) & 99 & 0 & 0 & 0 \\
\hline 17 & KF (2) & 100 & 0 & 0 & 0 \\
\hline $18^{c}$ & $\mathrm{NaBr}(2)$ & 99 & 0 & 0 & 0 \\
\hline $19^{d}$ & $\mathrm{Zn}(\mathrm{CN})_{2}(0.5)$ & 93 & 0 & 0 & 0 \\
\hline $20^{d}$ & $\mathrm{Zn}(\mathrm{CN})_{2}(0.5), \mathrm{NaBr}(2)$ & 0 & 38 & 45 & 3 \\
\hline $21^{d}$ & $\mathrm{NaCN}(1)$ & 89 & 0 & 0 & 0 \\
\hline $22^{d}$ & $\mathrm{NaCN}(1), \mathrm{NaBr}(2)$ & 93 & 0 & 0 & 0 \\
\hline
\end{tabular}

aDetermined by GC-MS using $n$-dodecane as internal standard.

bUsing $10 \mathrm{~mol} \% \mathrm{NiBr}_{2}(\mathrm{bpy}) \cdot \mathrm{xH}_{2} \mathrm{O}$.

without $\mathrm{NiBr}_{2}($ bpy $) \cdot \mathrm{xH}_{2} \mathrm{O}$.

awithout MPMN. 
Table S5. Evaluation of the reductive cyanation reaction for aryl chlorides

\begin{tabular}{|c|c|c|c|c|c|}
\hline NIE & $\begin{array}{r}\mathrm{MPMN} \\
\mathrm{NiBr}_{2}(\mathrm{bpy}) \cdot \times \mathrm{xH} \\
\text { add } \\
\mathrm{Zn}(2 \\
\mathrm{DMA}(0.2 \mathrm{M})\end{array}$ & $\begin{array}{l}20 \mathrm{~mol} \%) \\
\left.{ }^{\circ} \mathrm{C}\right) \\
{ }^{\circ} \mathrm{C}, 16 \mathrm{~h}\end{array}$ & $+4=$ & & \\
\hline $0.1 \mathrm{mmo}$ & & & & $\mathrm{Ar}-\mathrm{Ar}$ & \\
\hline Entry & Additive (equiv) & Remaining $\mathrm{ArCl}(\%)^{a}$ & Yield 2a $(\%)^{a}$ & Yield ArH (\%) ${ }^{a}$ & Yield $\operatorname{Ar}-\operatorname{Ar}(\%)^{a}$ \\
\hline 1 & none & 90 & 0 & 0 & 0 \\
\hline 2 & $\mathrm{NaBr}(2)$ & 14 & 53 & 18 & 0 \\
\hline 3 & $\mathrm{NaBr}(1)$ & 64 & 17 & 0 & 0 \\
\hline 4 & $\mathrm{NaBr}(4)$ & 21 & 55 & 16 & 0 \\
\hline 5 & $\mathrm{NaCl}(2)$ & 84 & 0 & 0 & 0 \\
\hline 6 & Nal (2) & 93 & 0 & 0 & 0 \\
\hline 7 & $\mathrm{KBr}(2)$ & 91 & 0 & 0 & 0 \\
\hline 8 & LiBr (2) & 87 & 0 & 0 & 0 \\
\hline 9 & TBAB (2) & 60 & 25 & 0 & 0 \\
\hline 10 & TBAC (2) & 87 & 0 & 0 & 0 \\
\hline 11 & TBAI (2) & 80 & 0 & 0 & 0 \\
\hline 12 & $\mathrm{MgBr}_{2}(1)$ & 83 & 7 & 0 & 0 \\
\hline 13 & $\mathrm{ZnBr}_{2}(1)$ & 89 & 0 & 0 & 0 \\
\hline
\end{tabular}

aDetermined by GC-MS using $n$-dodecane as internal standard. 


\section{Mechanistic and stoichiometric studies}

\section{C.1. Reactivity of ArZnBr with MPMN}

Scheme S1. Reactivity of ArZnBr with MPMN



Discussion: To evaluate the possibility of in situ arylzinc generation, arylzinc 3a was exposed to MPMN (1 equiv) in DMA/THF (2:1) for $16 \mathrm{~h}$ (Scheme S1). The reaction of 3a and MPMN alone resulted in no detectable formation of $\mathbf{2 a}$, and 3a was recovered solely as anisole (Table S6). Adding catalytic amounts of $\mathrm{NiBr}_{2}$ (bpy) or $\mathrm{NiBr}(\mathrm{bpy})$ resulted in some conversion to $\mathbf{2 a}$, however the major product in these cases was biaryl resulting from homocoupling of 3a. These product distributions are inconsistent with those observed in the catalytic reaction, suggesting that $\mathbf{3 a}$ is an unlikely reaction intermediate. The in situ generation of $\mathbf{3 a}$ under our reaction conditions is also unlikely, since exposing aryl bromide 1 a to reaction conditions without $\mathrm{Ni}$ results in little to no conversion of $1 \mathbf{a}$ (Table 1, Entry 6). Additionally, the oxidative addition of $\mathrm{Zn}(0)$ to aryl $\mathrm{C}-\mathrm{Br}$ bonds is typically quite challenging, especially for electron-rich substrates. ${ }^{7}$ Overall, in situ formation of an aryl-zinc seems unlikely to contribute significantly to product formation.

Table S6. Reactivity of ArZnBr with MPMN

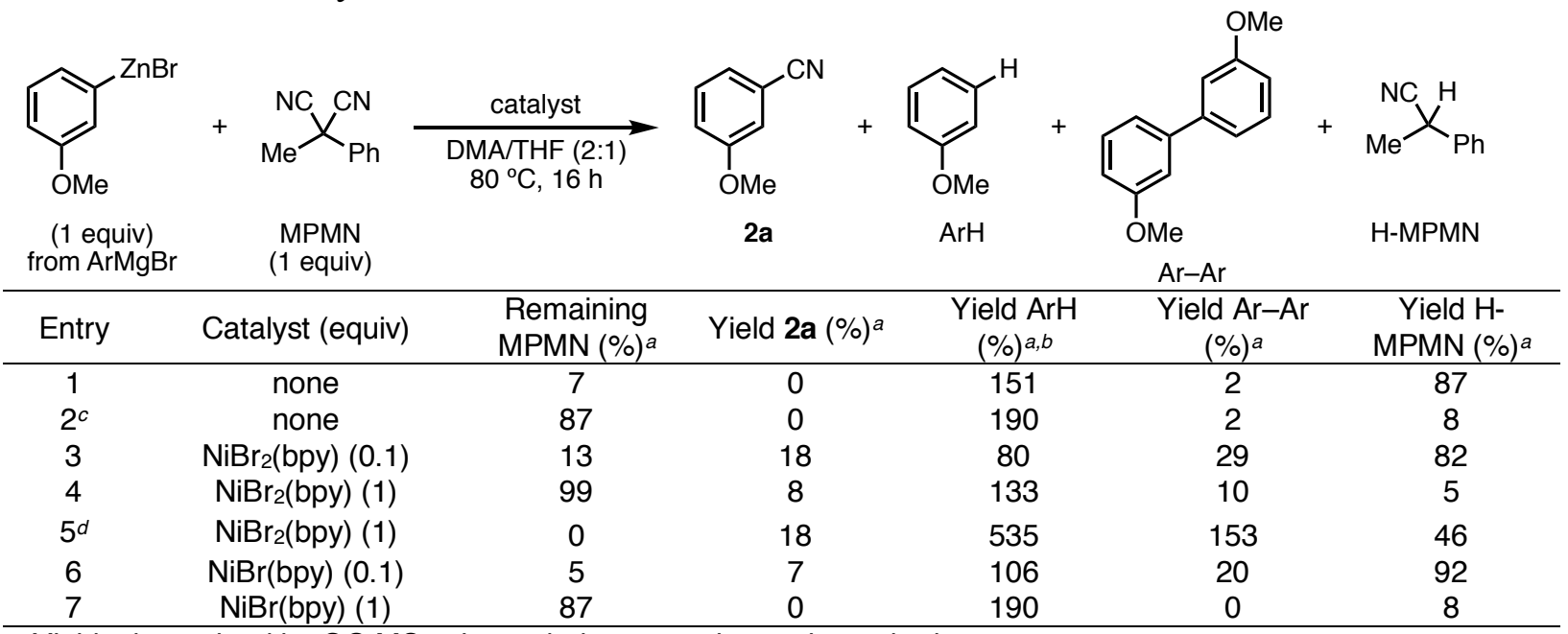

aYields determined by GC-MS using $n$-dodecane as internal standard.

${ }^{b}$ Arylzinc reagent was titrated with $\mathrm{I}_{2}$ before use; yields of $\mathrm{ArH}$ exceeding $100 \%$ are due to reduced product generated during Grignard formation.

cUsing ArZnBr from ArLi.

dUsing 10 equiv ArZnBr. 


\section{C.2. Reactivity Tables}

Table S7. Reactivity of post-oxidative addition complex 4 with MPMN

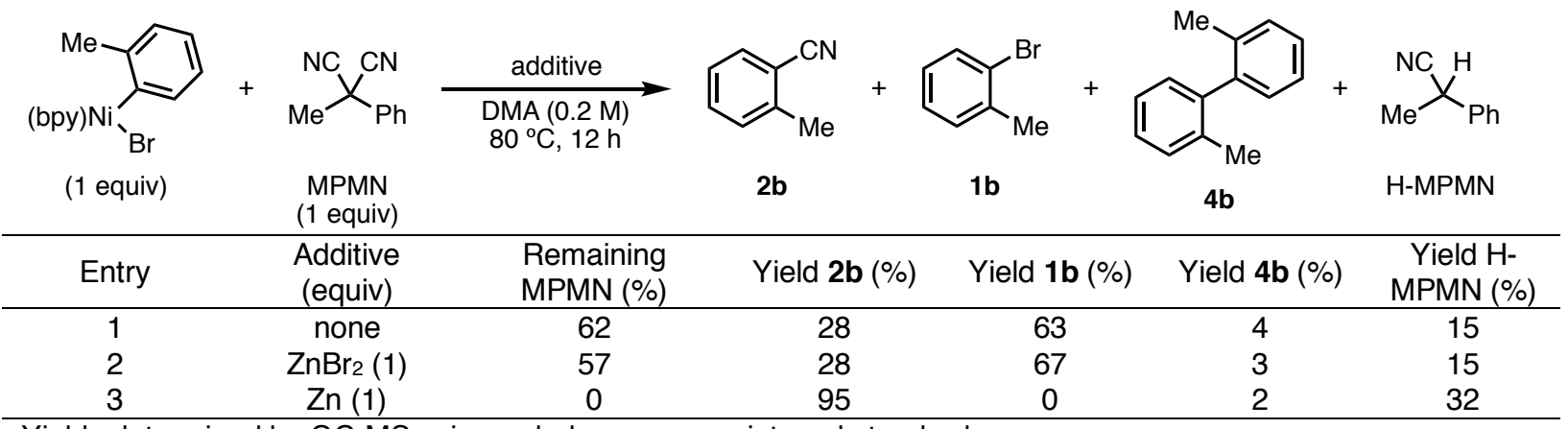

Yields determined by GC-MS using $n$-dodecane as an internal standard.

Note: Low yields of H-MPMN are due to inefficient protonolysis of $\alpha$-metalated H-MPMN.

Table S8. Transnitrilation in the presence of chloride additives

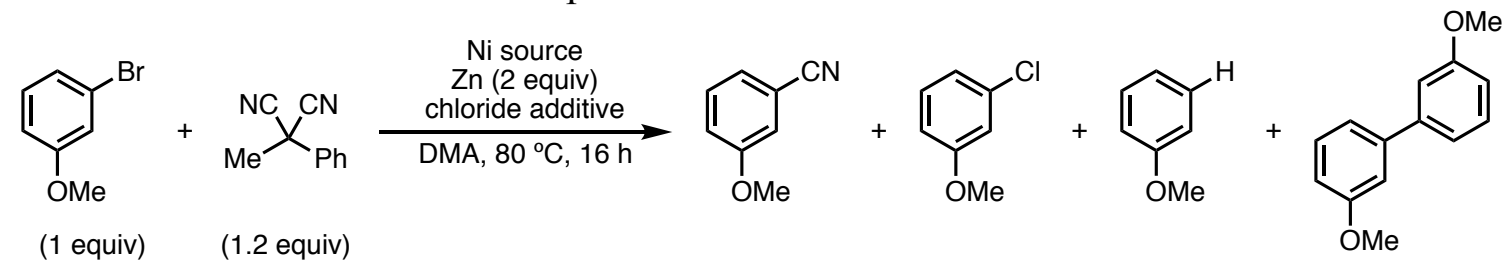

\begin{tabular}{cccccccc}
\hline Entry & $\begin{array}{c}\mathrm{Ni} \text { source } \\
\text { (equiv) }\end{array}$ & $\begin{array}{c}\text { additive } \\
\text { (equiv) }\end{array}$ & $\begin{array}{c}\text { Remaining } \\
\text { ArBr }\end{array}$ & Yield ArCN & Yield ArCl & Yield ArH & $\begin{array}{c}\text { Yield } \\
\text { Ar-Ar }\end{array}$ \\
\hline 1 & $\mathrm{NiBr}_{2}$ (bpy) (0.1) & none & 0 & 90 & 0 & 6 & 1 \\
2 & $\mathrm{NiBr}_{2}$ (bpy) (0.1) & $\mathrm{NaCl}(2)$ & 68 & 3 & 3 & 18 & 0 \\
3 & $\mathrm{NiBr}_{2}$ (bpy) (0.1) & $\mathrm{LiCl}(2)$ & 0 & 7 & 4 & 61 & 3 \\
4 & $\mathrm{NiBr}_{2}$ (bpy) (0.1) & $\mathrm{TBAC}(2)$ & 0 & 47 & 4 & 24 & 2 \\
5 & $\mathrm{NiBr}_{2}$ (bpy) (0.1) & $\mathrm{KCl}(2)$ & 65 & 2 & 3 & 24 & 0 \\
6 & $\mathrm{NiCl}_{2}$ (bpy) (1) & none & 3 & 38 & 39 & 4 & 2 \\
7 & $\mathrm{NiCl}_{2}$ (bpy) (1) & none & 0 & 37 & 39 & 4 & 3 \\
8 & $\mathrm{NiCl}_{2}$ (bpy) (1) & $\mathrm{NaBr}(2)$ & 0 & 53 & 0 & 14 & 8 \\
9 & $\mathrm{NiCl}_{2}$ (bpy) (1) & $\mathrm{TBAB}(2)$ & 0 & 43 & 0 & 14 & 16 \\
10 & $\mathrm{NiCl}_{2}$ (bpy) (1) & $\mathrm{TBAC}(2)$ & 0 & 47 & 0 & 10 & 19 \\
11 & $\mathrm{NiCl}_{2}$ (bpy) (1) & $\mathrm{LiBr}(2)$ & 0 & 40 & 0 & 12 & 20 \\
12 & $\mathrm{NiCl}_{2}$ (bpy) (0.4) & $\mathrm{NaBr}(2)$ & 0 & 57 & 0 & 14 & 7 \\
13 & $\mathrm{NiCl}_{2}$ (bpy) (0.2) & none & 0 & 73 & 7 & 16 & 3 \\
14 & $\mathrm{NiCl}_{2}$ (bpy) (0.2) & $\mathrm{NaBr}(2)$ & 0 & 75 & 0 & 8 & 5 \\
15 & $\mathrm{NiCl}_{2}$ (bpy) (0.1) & $\mathrm{None}$ & 0 & 78 & 7 & 12 & 2 \\
16 & $\mathrm{NiCl}_{2}$ (bpy) (0.1) & $\mathrm{NaBr}(2)$ & 75 & 7 & 0 & 14 & 0 \\
17 & $\mathrm{NiCl}_{2}$ (bpy) (0.1) & $\mathrm{TBAB}(2)$ & 100 & 0 & 0 & 0 & 0 \\
\hline
\end{tabular}

Yields determined by GC-MS using $n$-dodecane as an internal standard. 
Equation S1. Reaction of MPMN with $\mathrm{NiBr}_{2}($ bpy $) \bullet \mathrm{xH}_{2} \mathrm{O}$ and $\mathrm{Zn}(0)$ dust

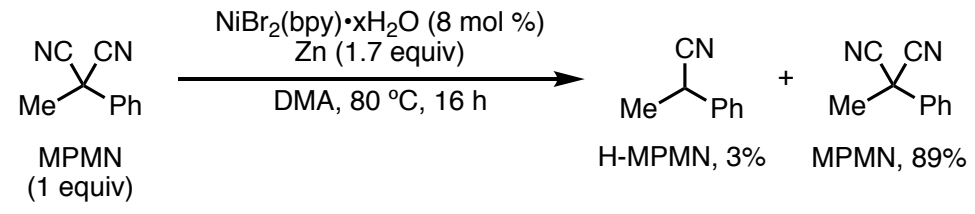

(Yields determined by GC-MS using $n$-dodecane as an internal standard.)

Table S9. Transnitrilation in the presence of $\mathrm{H}_{2} \mathrm{O}(0-1.0$ equiv)

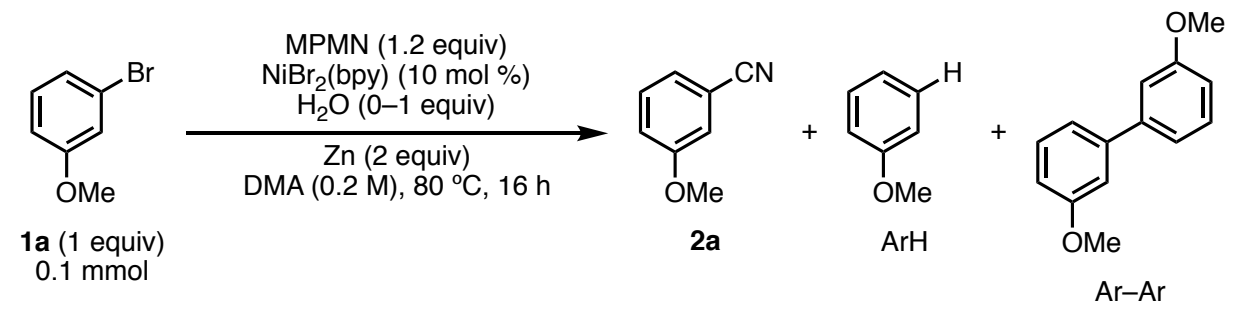

\begin{tabular}{cccccc}
\hline Entry & equiv $\mathrm{H}_{2} \mathrm{O}$ & Remaining $\mathrm{ArBr}(\%)^{a}$ & Yield 2a $(\%)^{a}$ & Yield ArH $(\%)^{a}$ & Yield $\operatorname{Ar}-\operatorname{Ar}(\%)^{a}$ \\
\hline 1 & 0 & 0 & 85 & 6 & 1 \\
2 & 0.1 & 0 & 67 & 18 & 3 \\
3 & 0.2 & 0 & 60 & 24 & 1 \\
4 & 0.4 & 0 & 63 & 22 & 0 \\
5 & 1.0 & 0 & 67 & 29 & 1 \\
\hline
\end{tabular}

aYields determined by GC-MS using $n$-dodecane as internal standard. 


\section{C.3. Testing the reaction mixture for cyanide using p-benzoquinone}

Quantification of cyanide levels at the end of a standard reaction using $p$-benzoquinone: ${ }^{8}$ To a 1dram vial was added $3.0 \mathrm{~mL}$ of a $0.34 \mathrm{mM} p$-benzoquinone stock solution in DMSO (1.0 mmol) and $0.10 \mathrm{~mL}$ of the appropriate aqueous test solution (see below), and the sample was allowed to stand at r.t. for $2 \mathrm{~h}$. The solution was transferred directly to a standard cuvette and was read at r.t. for fluorescence using a spectrofluorometer at excitation wavelength $400 \mathrm{~nm}$ and emission wavelengths $450-500 \mathrm{~nm}$. The following aqueous samples were analyzed: (a) Blank, $\mathrm{H}_{2} \mathrm{O}$; (b) $600 \mu \mathrm{g}$ Control, $1.02 \times 10^{-4} \mathrm{M} \mathrm{Zn}(\mathrm{CN})_{2}$; (c) $60 \mu \mathrm{g}$ Control, $1.02 \times 10^{-5} \mathrm{M} \mathrm{Zn}(\mathrm{CN})_{2}$; (d) Reaction Mixture, taken from the aqueous layer of a standard reaction after workup, extraction with EtOAc $(\times 3)$, and filtration of the aqueous layer over Celite.

\section{Figure S1. $p$-Benzoquinone test fluorescence data}

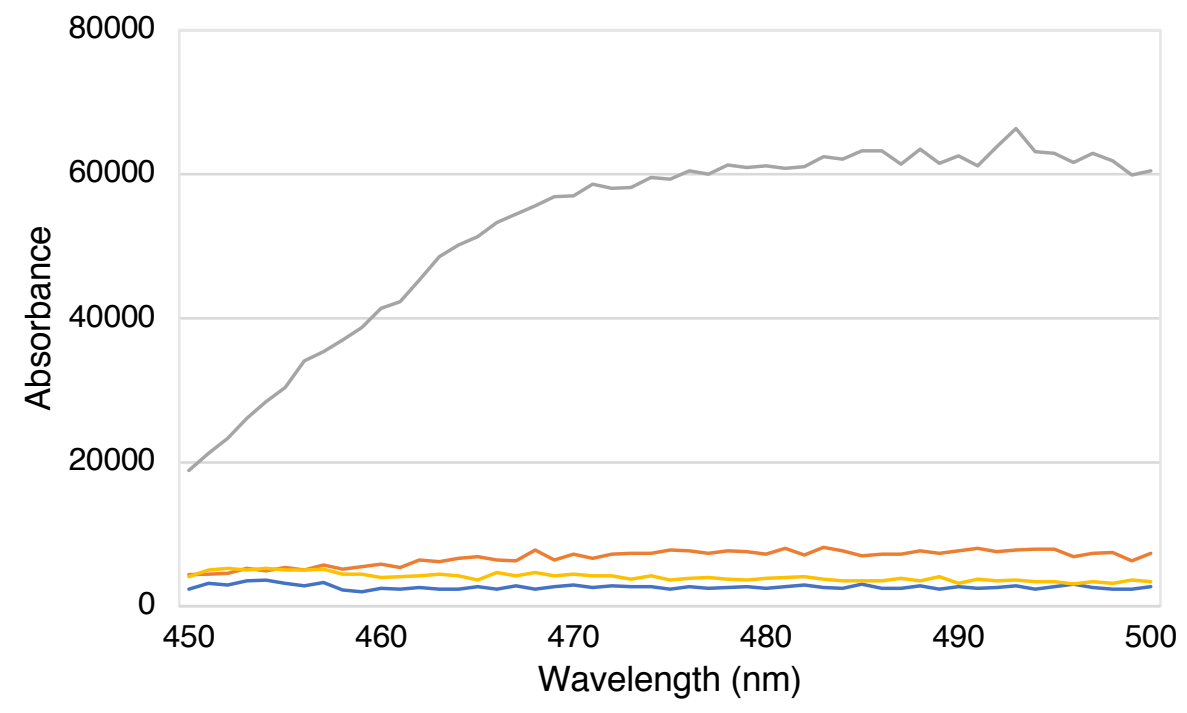

(a) Blank

(b) $600 \mu \mathrm{g}$ Control

(c) $60 \mu \mathrm{g}$ Control

(d) Reaction Mixture

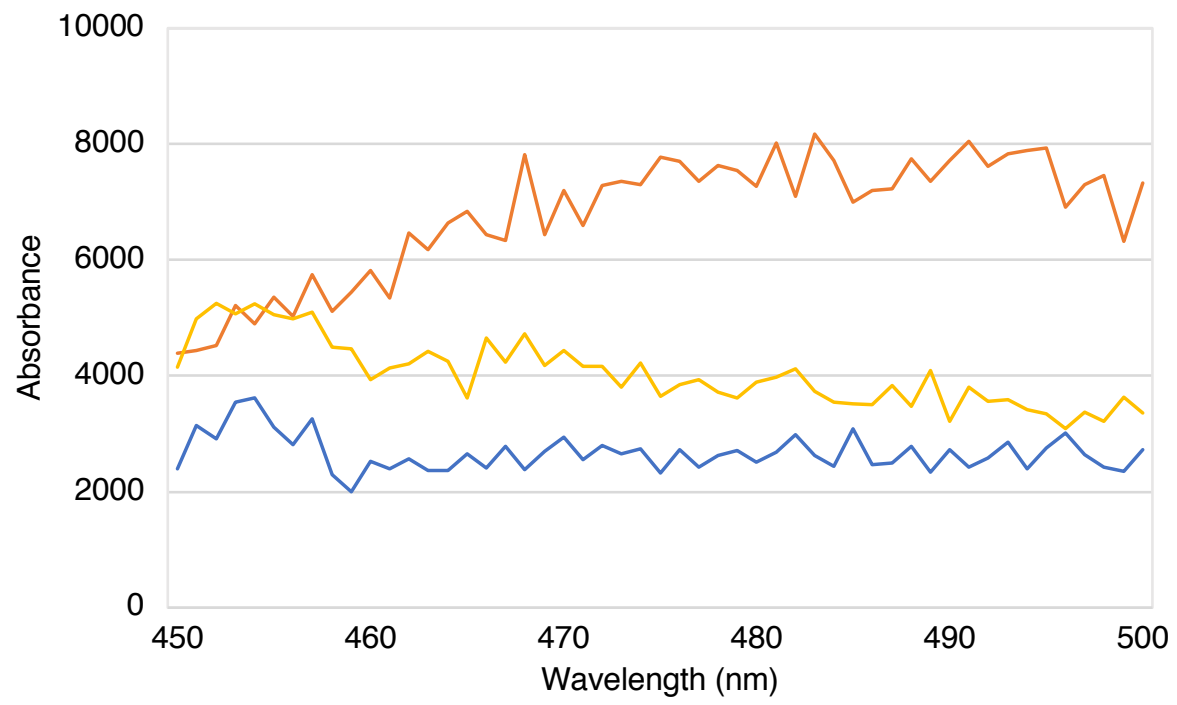

(a) Blank

(c) $60 \mu \mathrm{g}$ Control

(d) Reaction Mixture 


\section{Preparation of catalysts and other reagents}

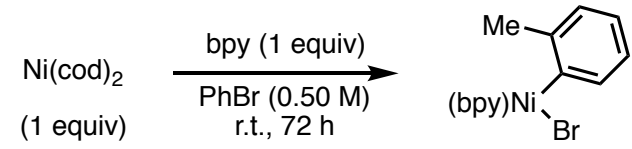

(bpy)NiBr(o-Tol) (4): To a flame-dried scintillation vial with a stir bar was added 2,2'bipyridine $(0.16 \mathrm{~g}, 1.0 \mathrm{mmol}, 1.0$ equiv), and the vial was brought into a glovebox. 2Bromotoluene $(2.0 \mathrm{~mL}, 0.50 \mathrm{M})$ was added, and the solution was stirred to dissolve 2,2'bipyridine. While stirring, $\mathrm{Ni}(\mathrm{cod})_{2}(0.28 \mathrm{~g}, 1.0 \mathrm{mmol}, 1.0$ equiv) was added. The reaction was stirred at r.t. for $72 \mathrm{~h}$. The reaction was stopped by addition of pentane (ca. $10 \mathrm{~mL}$ ). The solution was decanted, and the precipitate was thoroughly washed with pentane $(5 \times 10 \mathrm{~mL})$ and dried under vacuum for $12 \mathrm{~h}$ to yield 4 as an orange-red solid $(0.35 \mathrm{~g}, 0.91 \mathrm{mmol}, 91 \%) .4$ was stored at $-20^{\circ} \mathrm{C}$ in a glovebox. Crystals suitable for X-ray diffraction (see Section $\mathrm{H}$ ) were grown by $\mathrm{THF} /$ pentane vapour diffusion for $7 \mathrm{~d}$ at r.t. $(0.50 \mathrm{~mL}$ saturated solution in $\mathrm{THF}+0.50 \mathrm{~mL}$ pentane). The analytical data is consistent with similar Ni(II) intermediates: ${ }^{9}{ }^{1} \mathbf{H}$ NMR (500 $\mathrm{MHz}$, acetone- $\left.\delta_{6}, 298 \mathrm{~K}\right): \delta_{\mathrm{H}} 9.42(\mathrm{~d}, J=5.5 \mathrm{~Hz}, 1 \mathrm{H}), 8.41-8.29(\mathrm{~m}, 2 \mathrm{H}), 8.25-8.11(\mathrm{~m}, 2 \mathrm{H})$, $7.71(\mathrm{t}, J=6.7 \mathrm{~Hz}, 1 \mathrm{H}), 7.52(\mathrm{dd}, J=7.4,1.5 \mathrm{~Hz}, 1 \mathrm{H}), 7.36(\mathrm{t}, J=5.3 \mathrm{~Hz}, 1 \mathrm{H}), 7.16(\mathrm{~d}, J=5.8$ $\mathrm{Hz}, 1 \mathrm{H}), 6.79-6.64(\mathrm{~m}, 3 \mathrm{H}), 3.01$ (s, 3H) ppm.

Figure S2. ORTEP structure of complex 4

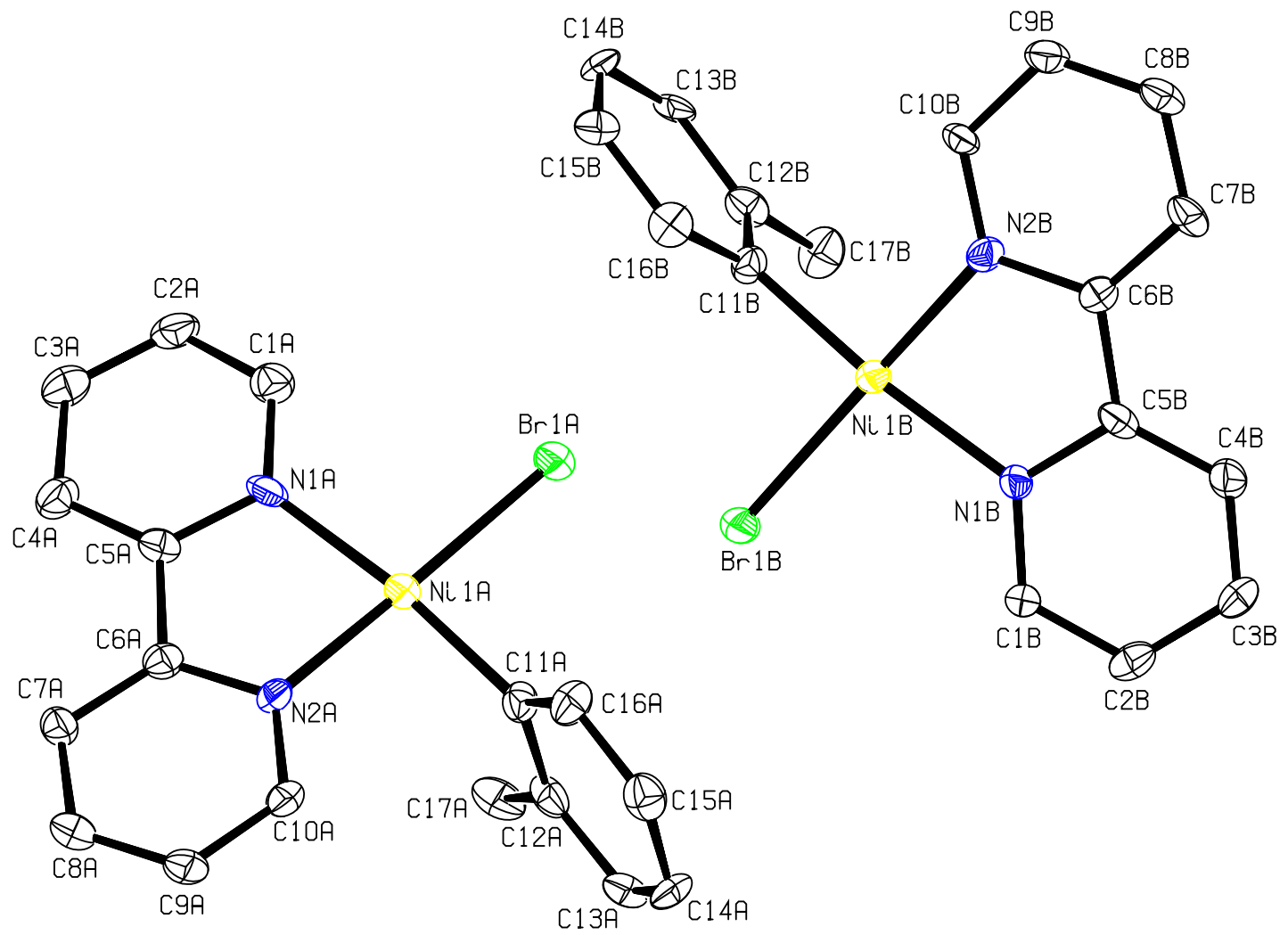




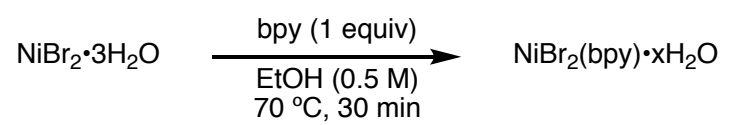

$\mathrm{NiBr}_{2}$ (bpy) $\bullet \mathbf{x H}_{2} \mathbf{O}$ : To a $500-\mathrm{mL}$ flask with a stir bar and reflux condenser was added $\mathrm{NiBr}_{2} \bullet 3 \mathrm{H}_{2} \mathrm{O}(27.3 \mathrm{~g}, 100 \mathrm{mmol}, 1.00$ equiv) and $\mathrm{EtOH}(200 \mathrm{~mL}, 0.50 \mathrm{M})$. The solution was stirred at r.t. until $\mathrm{NiBr}_{2} \bullet 3 \mathrm{H}_{2} \mathrm{O}$ was completely dissolved. 2,2'-Bipyridine (15.6 g, $100 \mathrm{mmol}, 1.0$ equiv) was added and the reaction was heated at reflux at $70^{\circ} \mathrm{C}$ for $30 \mathrm{~min}$. The reaction was cooled to r.t. and concentrated to a volume of ca. $50 \mathrm{~mL}$. The mixture was filtered using a fritted funnel and the precipitate was washed with acetone $(\times 3)$ and dried under high vacuum for $8 \mathrm{~h}$ to yield $\mathrm{NiBr}_{2}$ (bpy) $\bullet \mathrm{xH}_{2} \mathrm{O}$ as a pale green powder $(25.8 \mathrm{~g}, 68.9 \mathrm{mmol}, 69 \%) . \mathrm{NiBr}_{2}($ bpy $) \bullet \mathrm{xH}_{2} \mathrm{O}$ was stored in a dessicator open to air and was weighed, assuming the MW for anhydrous $\mathrm{NiBr}_{2}(\mathrm{bpy})$. Note: Small changes in stoichiometry or the order of additions for this procedure yielded precatalysts that performed irreproducibly.

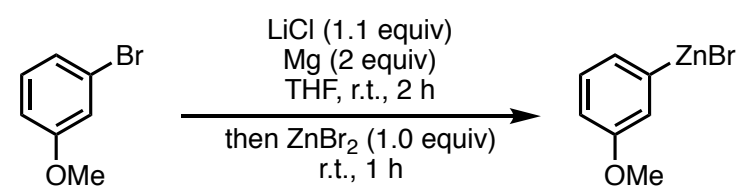

(3-Methoxyphenyl)zinc(II) bromide (from ArMgBr): To a 25-mL flask with a stir bar was added $\mathrm{Mg}(0)$ turnings ( $0.48 \mathrm{~g}, 20 \mathrm{mmol}, 2.0$ equiv) and lithium chloride ( $0.46 \mathrm{~g}, 11 \mathrm{mmol}, 1.1$ equiv). The flask was flame-dried and cooled under vacuum. The flask was backfilled with $\mathrm{N}_{2}$ and THF $(10 \mathrm{~mL})$ was added. The $\mathrm{Mg}(0)$ turnings were activated with 1,2-dibromoethane (ca. $0.050 \mathrm{~mL})$ and 3-bromoanisole $(1.3 \mathrm{~mL}, 10 \mathrm{mmol}, 1.0$ equiv) was added portionwise over 30 min. The reaction was stirred at r.t. for another $1 \mathrm{~h}$ to yield a solution of 3methoxyphenylmagnesium bromide. To a separate flame-dried $25-\mathrm{mL}$ flask with a stir bar was added zinc(II) bromide $(2.3 \mathrm{~g}, 10 \mathrm{mmol}, 1.0$ equiv) and THF $(10 \mathrm{~mL})$. The resulting solution was stirred at r.t. for $15 \mathrm{~min}$. Then, the solution of 3-methoxyphenylmagnesium bromide in THF was added via syringe, and the reaction was stirred at r.t. for $1 \mathrm{~h}$. The solution was allowed to settle for $1 \mathrm{~h}$ to yield (3-methoxyphenyl)zinc(II) bromide as a grey solution in THF. The solution was titrated with $\mathrm{I}_{2}$ and was found to have a concentration of $0.33 \mathrm{M}(6.6 \mathrm{mmol}, 66 \%$ yield $)$.

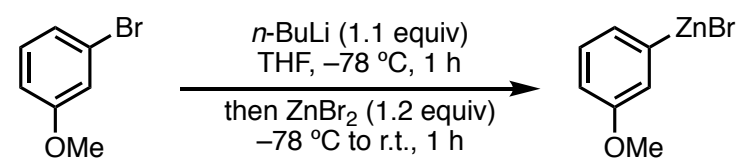

(3-Methoxyphenyl)zinc(II) bromide (from ArLi): To a flame-dried 25-mL flask with a stir bar was added 3-bromoanisole (1.3 mL, $10 \mathrm{mmol}, 1.0$ equiv) and THF (10 mL, 1.0 M). The solution was cooled to $-78{ }^{\circ} \mathrm{C}$ and $n$-butyllithium $(6.9 \mathrm{~mL}$ of a $1.6 \mathrm{M}$ solution in hexanes, $11 \mathrm{mmol}, 1.1$ equiv) was added dropwise over $10 \mathrm{~min}$. The solution was stirred at $-78^{\circ} \mathrm{C}$ for $1 \mathrm{~h}$. The flask was briefly opened to air and zinc(II) bromide ( $2.7 \mathrm{~g}, 12 \mathrm{mmol}, 1.2$ equiv) was added at once. The reaction was stirred at $-78^{\circ} \mathrm{C}$ for $15 \mathrm{~min}$, then at r.t. for $1 \mathrm{~h}$. THF $(8.0 \mathrm{~mL})$ was added to emulsify the two-layer mixture, yielding (3-methoxyphenyl)zinc(II) bromide as a colourless solution in THF. The solution was titrated with $\mathrm{I}_{2}$ and was found to have a concentration of $0.20 \mathrm{M}(5.0 \mathrm{mmol}, 50 \%$ yield$)$.

Zn dust (activated): ${ }^{5}$ Zinc was stirred for $2 \min$ in $10 \%$ aq. $\mathrm{HCl}$ and the solution was filtered through a fritted funnel. Zinc chunks were broken up and washed with $\mathrm{H}_{2} \mathrm{O}$ until the $\mathrm{pH}$ of the 
filtrate turned neutral $(\times 3)$, then with acetone $(\times 3)$. Zinc dust was dried under vacuum for $8 \mathrm{~h}$ before use. 


\section{E. Procedures for the preparation of benzonitriles}

General Procedure A: Synthesis of benzonitriles

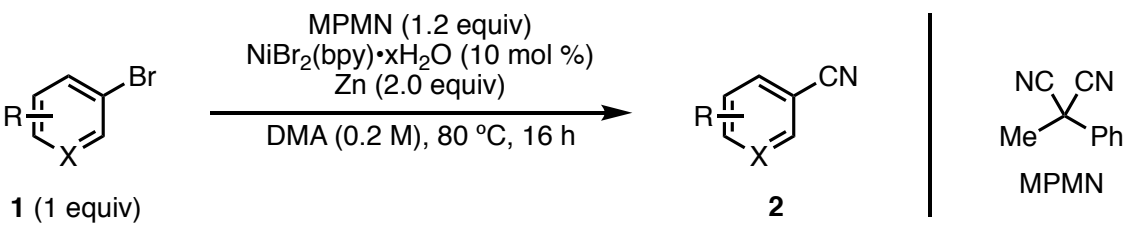

Caution: Depending on the reductant, ligand, and nickel catalyst used, decyanation of excess MPMN was sometimes observed, implying formation of cyanide salts in situ under those conditions. As a precaution, all reactions were handled under basic conditions and were disposed of appropriately.

To a flame-dried 8-mL culture tube with stir bar was added zinc(0) dust (52 $\mathrm{mg}, 0.80 \mathrm{mmol}, 2.0$ equiv), aryl halide (if a solid; $0.40 \mathrm{mmol}, 1.0$ equiv), and $\mathrm{NiBr}_{2}(\mathrm{bpy}) \cdot \mathrm{xH}_{2} \mathrm{O}(15 \mathrm{mg}, 0.040 \mathrm{mmol}$, $10 \mathrm{~mol} \%)$. The tube was sealed with a size-19 septum and electrical tape and was evacuated and backfilled with $\mathrm{N}_{2}(\times 3)$. 2-Methyl-2-phenylmalononitrile $(2.0 \mathrm{~mL}$ of a $0.24 \mathrm{M}$ stock solution in DMA, $0.48 \mathrm{mmol}, 1.2$ equiv) was added at r.t. If the aryl halide was an oil, aryl halide was then added $(0.40 \mathrm{mmol}, 1.0$ equiv). The reaction was stirred at r.t. for $2 \mathrm{~min}$ then was put into an oil bath at $80^{\circ} \mathrm{C}$ and was stirred for $16 \mathrm{~h}$. The reaction was cooled to r.t., quenched with sat. aq. $\mathrm{NaHCO}_{3}$, and $n$-dodecane ( $91 \mu \mathrm{L}, 0.40 \mathrm{mmol}, 1.0$ equiv) was added as an internal standard for GC-MS analysis. The solution was extracted with EtOAc $(\times 3)$ and the organic fractions were combined, filtered over a plug of $\mathrm{MgSO}_{4}$ and Celite, and concentrated. The crude residue was purified by flash column chromatography to yield the desired benzonitrile. 
Figure S3. Representative unsuccessful substrates

a) Substrates with low conversion:<smiles>Brc1cncnc1</smiles><smiles>Brc1cccnc1</smiles><smiles>COc1ccc(Br)cc1[N+](=O)[O-]</smiles><smiles>Cn1cc(Br)ccc1=O</smiles><smiles>Pn1cc(Br)cn1</smiles><smiles>Brc1ccc(C(c2ccc(Br)cc2)n2cncn2)cc1</smiles><smiles>Oc1ccc(Br)cc1</smiles><smiles>Oc1ccc2cc(Br)ccc2c1</smiles><smiles>Nc1ccc(Br)cc1</smiles><smiles>Cc1cc(C)c(Br)c(C)c1</smiles>

b) Substrates that gave mixtures<smiles>Brc1nccc2ccccc12</smiles><smiles>Clc1ncc(Br)cn1</smiles><smiles></smiles><smiles>O=C(Nc1ccc(Br)cc1)c1ccccc1</smiles><smiles>Sc1ncc(Br)cn1</smiles><smiles>O=C1CCc2ccc(Br)cc21</smiles>

c) Substrates with incompatible functional groups<smiles>Fc1ccc(Br)c2ccccc12</smiles>

$$
\mathrm{S}_{\mathrm{N}} \mathrm{Ar} \text { of the }
$$
4-fluorobenzonitrile<smiles>CCC[Se]c1ccc(Br)c2ccccc12</smiles>

$\mathrm{C}-\mathrm{S}$ to $\mathrm{C}-\mathrm{H}$ reduction<smiles>Fc1ccc(Br)cn1</smiles>

low conv. and $\mathrm{C}-\mathrm{F}$ functionalization<smiles>Brc1cnc(Oc2ccc(I)cc2)nc1</smiles>

C-I functionalization

d) Substrates with potecting groups that were cleaved during the reaction<smiles>O=C(O)c1ccc(Br)cc1-n1cc(Br)cn1</smiles> 


\section{E.1. Synthesis of benzonitriles}

Table S10. Supplemental scope table

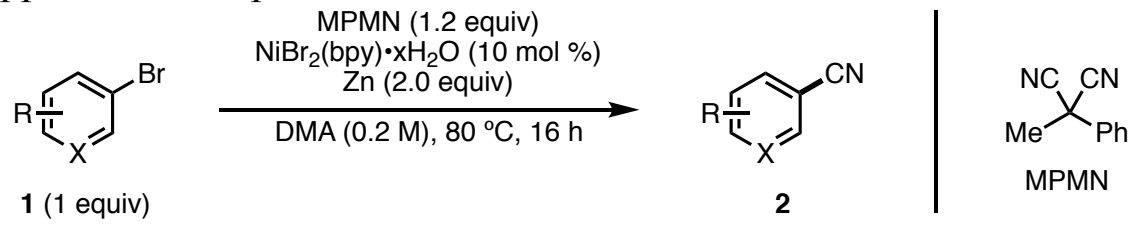<smiles>N#Cc1ccccc1[N+](=O)[O-]</smiles>

2ah, $41 \%$<smiles>C=Cc1cccc(C#N)c1</smiles>

2ai, $26 \%$<smiles>N#Cc1ccc2cc(OC(=O)c3ccccc3)ccc2c1</smiles>

2aj, $45 \%$<smiles>CC(C)(C)O[Si](C)(C)Oc1ccc(C#N)cc1</smiles>

2ak, $24 \%$<smiles>N#Cc1cccc(C#N)c1</smiles>

2al, $54 \%$<smiles>N#Cc1ccc2c(c1)COC2=O</smiles>

2am, 39\%<smiles>COC(c1ccc2c(c1)OCO2)c1ccc(C#N)s1</smiles>

2an, $32 \%$<smiles>N#Cc1cnn(Cc2ccccc2)c1</smiles>

2ao, $22 \%$<smiles>CN1C(=O)C(=O)c2cc(C#N)ccc21</smiles>

2ap, $19 \%$<smiles>N#Cc1cnc2ccccc2c1</smiles>

2aq, $33 \%$<smiles>COc1ccc(C#N)cn1</smiles>

2ar, $36 \%$<smiles>Cc1ccc(C#N)cc1C</smiles>

3,4-Dimethylbenzonitrile (2b) (CAS: 22884-95-3): According to General Procedure A, 2b was prepared using the following amounts of reagent: $\mathrm{NiBr}_{2}$ (bpy) $\bullet \mathrm{xH}_{2} \mathrm{O}(15 \mathrm{mg}, 0.040 \mathrm{mmol}, 10 \mathrm{~mol}$ $\%), \mathrm{Zn}(0)$ dust (52 mg, $0.80 \mathrm{mmol}, 2.0$ equiv), 4-bromo-1,2-dimethylbenzene (54 $\mu \mathrm{L}, 0.40$ mmol, 1.0 equiv), and MPMN (2.0 mL of a $0.24 \mathrm{M}$ stock solution in DMA, $0.48 \mathrm{mmol}, 1.2$ equiv). The crude residue was purified by flash column chromatography (gradient of $0-5 \%$ EtOAc/hexanes) to yield $\mathbf{2 b}$ as a white solid (trial 1: $39 \mathrm{mg}, 0.297 \mathrm{mmol}, 74 \%$ : trial 2: $33 \mathrm{mg}$, $0.252 \mathrm{mmol}, 63 \%)$. Analytical data: ${ }^{10}{ }^{1} \mathrm{H}$ NMR $\left(500 \mathrm{MHz}, \mathrm{CDCl}_{3}, 298 \mathrm{~K}\right): \delta_{\mathrm{H}} 7.43-7.33(\mathrm{~m}$, 2H), 7.24-7.16 (m, 1H), $2.31(\mathrm{~s}, 3 \mathrm{H}), 2.28(\mathrm{~s}, 3 \mathrm{H}) \mathrm{ppm} ;{ }^{13} \mathbf{C} \mathbf{N M R}\left(125 \mathrm{MHz}, \mathrm{CDCl}_{3}, 298 \mathrm{~K}\right): \delta_{\mathrm{C}}$ 142.6, 138.0, 133.0, 130.4, 129.8, 119.4, 109.6, 20.2, 20.0 ppm; $\mathbf{R}_{\mathbf{f}}(9: 1$ hexanes/EtOAc; $\left.\mathrm{UV} / \mathrm{KMnO}_{4}\right): 0.82$.<smiles>Cc1ccccc1C#N</smiles>

2-Methylbenzonitrile (2ah) (CAS: 529-19-1): According to General Procedure A, the product was prepared using the following amounts of reagent: $\mathrm{NiBr}_{2}$ (bpy) $\bullet \mathrm{xH}_{2} \mathrm{O}(15 \mathrm{mg}, 0.040 \mathrm{mmol}, 10$ mol \%), $\mathrm{Zn}(0)$ dust (52 mg, $0.80 \mathrm{mmol}, 2.0$ equiv), 2-bromotoluene (48 $\mu \mathrm{L}, 0.40 \mathrm{mmol}, 1.0$ equiv), and MPMN (2.0 mL of a $0.24 \mathrm{M}$ stock solution in DMA, $0.48 \mathrm{mmol}, 1.2$ equiv). The crude residue was purified by flash column chromatography (gradient of 0-10\% EtOAc/hexanes) to yield the product as a colourless oil $(19 \mathrm{mg}, 0.162 \mathrm{mmol}, 41 \%)$. Analytical data: ${ }^{11} \mathbf{1} \mathbf{H}$ NMR $\left(400 \mathrm{MHz}, \mathrm{CDCl}_{3}, 298 \mathrm{~K}\right): \delta_{\mathrm{H}} 7.63-7.55(\mathrm{~m}, 1 \mathrm{H}), 7.51-7.42(\mathrm{~m}, 1 \mathrm{H}), 7.36-7.22(\mathrm{~m}, 2 \mathrm{H}), 2.55(\mathrm{~s}$, $3 \mathrm{H}) \mathrm{ppm} ;{ }^{13} \mathbf{C} \mathbf{N M R}\left(100 \mathrm{MHz}, \mathrm{CDCl}_{3}, 298 \mathrm{~K}\right): \delta_{\mathrm{C}} 142.0,132.6,132.5,130.2,126.2,118.1$, 112.8, 20.5 ppm; $\mathbf{R}_{\mathbf{f}}\left(9: 1\right.$ hexanes/EtOAc; $\left.\mathrm{UV} / \mathrm{KMnO}_{4}\right): 0.48$. 
<smiles>N#Cc1ccc2ccccc2c1</smiles>

2-Naphthonitrile (2c) (CAS: 613-46-7): According to General Procedure A, the product was prepared using the following amounts of reagent: $\mathrm{NiBr}_{2}$ (bpy) $\bullet \mathrm{xH}_{2} \mathrm{O}(15 \mathrm{mg}, 0.040 \mathrm{mmol}, 10 \mathrm{~mol}$ $\%$ ), $\mathrm{Zn}(0)$ dust (52 mg, $0.80 \mathrm{mmol}, 2.0$ equiv), 2-bromonaphthalene (68 mg, $0.40 \mathrm{mmol}, 1.0$ equiv), and MPMN (2.0 mL of a $0.24 \mathrm{M}$ stock solution in DMA, $0.48 \mathrm{mmol}, 1.2$ equiv). The crude residue was purified by flash column chromatography (gradient of 0-20\% EtOAc/hexanes) to yield the product as a white solid (trial 1: $51 \mathrm{mg}, 0.333 \mathrm{mmol}, 83 \%$; trial 2: $46 \mathrm{mg}, 0.300$ mmol, 75\%). Analytical data: ${ }^{10}{ }^{1} \mathbf{H}$ NMR (400 MHz, $\left.\mathrm{CDCl}_{3}, 298 \mathrm{~K}\right): \delta_{\mathrm{H}} 8.25-8.20(\mathrm{~m}, 1 \mathrm{H})$, 7.94-7.86 (m, 3H), 7.68-7.57 (m, 3H) ppm; ${ }^{13} \mathbf{C} \mathbf{N M R}\left(100 \mathrm{MHz}, \mathrm{CDCl}_{3}, 298 \mathrm{~K}\right): \delta_{\mathrm{C}} 134.8$, $134.3,132.4,129.3,129.2,128.5,128.2,127.8,126.5,119.4,109.5$ ppm; $\mathbf{R}_{\mathbf{f}}(9: 1$ hexanes/EtOAc; UV): 0.62.<smiles>CC(C)(C)c1ccc(C#N)cc1</smiles>

4-(tert-Butyl)benzonitrile (2d) (CAS: 4210-32-6): According to General Procedure A, the product was prepared using the following amounts of reagent: $\mathrm{NiBr}_{2}(\mathrm{bpy}) \bullet \mathrm{xH}_{2} \mathrm{O}(15 \mathrm{mg}, 0.040$ mmol, $10 \mathrm{~mol} \%$ ), $\mathrm{Zn}(0)$ dust (52 mg, $0.80 \mathrm{mmol}, 2.0$ equiv), 1-bromo-4-tert-butylbenzene (69 $\mu \mathrm{L}, 0.40 \mathrm{mmol}, 1.0$ equiv), and MPMN (2.0 mL of a $0.24 \mathrm{M}$ stock solution in DMA, $0.48 \mathrm{mmol}$, 1.2 equiv). The crude residue was purified by flash column chromatography (gradient of $0-10 \%$ EtOAc/hexanes) to yield the product as a colourless oil (49 $\mathrm{mg}, 0.308 \mathrm{mmol}, 77 \%)$. Analytical data: ${ }^{10}{ }^{1} \mathbf{H}$ NMR $\left(500 \mathrm{MHz}, \mathrm{CDCl}_{3}, 298 \mathrm{~K}\right): \delta_{\mathrm{H}} 7.61-7.56(\mathrm{~m}, 2 \mathrm{H}), 7.50-7.45(\mathrm{~m}, 2 \mathrm{H}), 1.33(\mathrm{~s}$, 9H) ppm; ${ }^{13} \mathbf{C}$ NMR $\left(125 \mathrm{MHz}, \mathrm{CDCl}_{3}, 298 \mathrm{~K}\right): \delta_{\mathrm{C}} 156.6,132.0,126.2,119.2,109.3,32.3,30.9$ ppm; $\mathbf{R}_{\mathbf{f}}(9: 1$ hexanes/EtOAc): 0.63.<smiles>COc1ccc(C#N)cc1</smiles>

4-Methoxybenzonitrile (2e) (CAS: 874-90-8): According to General Procedure A, the product was prepared using the following amounts of reagent: $\mathrm{NiBr}_{2}$ (bpy) $\mathrm{xH}_{2} \mathrm{O}(15 \mathrm{mg}, 0.040 \mathrm{mmol}, 10$ mol \%), $\mathrm{Zn}(0)$ dust (52 mg, $0.80 \mathrm{mmol}, 2.0$ equiv), 4-bromoanisole (50 $\mu \mathrm{L}, 0.40 \mathrm{mmol}, 1.0$ equiv), and MPMN (2.0 mL of a $0.24 \mathrm{M}$ stock solution in DMA, $0.48 \mathrm{mmol}, 1.2$ equiv). The crude residue was purified by flash column chromatography (gradient of 0-20\% EtOAc/hexanes) to yield the product as a white solid (48 mg, $0.361 \mathrm{mmol}, 90 \%)$. Analytical data: ${ }^{10}{ }^{1} \mathbf{H}$ NMR (400 $\left.\mathrm{MHz}, \mathrm{CDCl}_{3}, 298 \mathrm{~K}\right): \delta_{\mathrm{H}} 7.63-7.53(\mathrm{~m}, 2 \mathrm{H}), 7.00-6.88(\mathrm{~m}, 2 \mathrm{H}), 3.85(\mathrm{~s}, 3 \mathrm{H}) \mathrm{ppm} ;{ }^{13} \mathbf{C} \mathbf{N M R}$ $\left(100 \mathrm{MHz}, \mathrm{CDCl}_{3}, 298 \mathrm{~K}\right): \delta_{\mathrm{C}} 163.0,134.1,119.3,114.9,104.1,55.6 \mathrm{ppm} ; \mathrm{R}_{\mathrm{f}}(9: 1$ hexanes/EtOAc; UV): 0.27.<smiles>N#Cc1ccc(OP)cc1</smiles>

4-Isopropoxybenzonitrile (2f) (CAS: 91949-95-0): According to General Procedure A, the product was prepared using the following amounts of reagent: $\mathrm{NiBr}_{2}(\mathrm{bpy}) \bullet \mathrm{xH}_{2} \mathrm{O}(15 \mathrm{mg}, 0.040$ mmol, $10 \mathrm{~mol} \%$ ), $\mathrm{Zn}(0)$ dust ( $52 \mathrm{mg}, 0.80 \mathrm{mmol}, 2.0$ equiv), 1-bromo-4-isopropoxybenzene (86 $\mathrm{mg}, 0.40 \mathrm{mmol}, 1.0$ equiv), and MPMN (2.0 mL of a $0.24 \mathrm{M}$ stock solution in DMA, $0.48 \mathrm{mmol}$, 1.2 equiv). The crude residue was purified by flash column chromatography (gradient of 0-20\% 
acetone/hexanes) to yield $\mathbf{2 f}$ as a white solid (trial 1: $63 \mathrm{mg}, 0.391 \mathrm{mmol}, 98 \%$; trial 2: $57 \mathrm{mg}$, 0.354, 89\%). Analytical data: ${ }^{12}{ }^{1} \mathbf{H}$ NMR (500 MHz, $\left.\mathrm{CDCl}_{3}, 298 \mathrm{~K}\right): \delta_{\mathrm{H}} 7.59-7.52(\mathrm{~m}, 2 \mathrm{H})$, 6.93-6.88 (m, 2H), 4.61 (sept, $J=6.1 \mathrm{~Hz}, 1 \mathrm{H}), 1.36(\mathrm{~d}, J=6.1 \mathrm{~Hz}, 6 \mathrm{H}) \mathrm{ppm} ;{ }^{13} \mathbf{C}$ NMR $(125$ $\left.\mathrm{MHz}, \mathrm{CDCl}_{3}, 298 \mathrm{~K}\right): \delta_{\mathrm{C}} 161.3,134.0,119.3,116.0,103.3,70.4,21.8$ ppm; $\mathbf{R}_{\mathbf{f}}(9: 1$ hexanes/EtOAc; UV): 0.36.<smiles>N#Cc1ccc(Oc2ccccc2)cc1</smiles>

4-Phenoxybenzonitrile (2g) (CAS: 3096-81-9): According to General Procedure A, the product was prepared using the following amounts of reagent: $\mathrm{NiBr}_{2}$ (bpy) $\mathrm{xH}_{2} \mathrm{O}(15 \mathrm{mg}, 0.040 \mathrm{mmol}, 10$ mol \%), Zn(0) dust (52 mg, $0.80 \mathrm{mmol}, 2.0$ equiv), 1-bromo-4-phenoxybenzene (S2) (0.10 g, $0.40 \mathrm{mmol}, 1.0$ equiv), and MPMN (2.0 $\mathrm{mL}$ of a $0.24 \mathrm{M}$ stock solution in DMA, $0.48 \mathrm{mmol}, 1.2$ equiv). The crude residue was purified by flash column chromatography (gradient of $0-15 \%$ acetone/hexanes) to yield $2 \mathrm{~g}$ as a white solid (trial 1: $51 \mathrm{mg}, 0.261 \mathrm{mmol}, 65 \%$; trial 2: $57 \mathrm{mg}$, $0.292 \mathrm{mmol}, 73 \%)$. Analytical data: ${ }^{13}{ }^{1} \mathrm{H}$ NMR $\left(500 \mathrm{MHz}, \mathrm{CDCl}_{3}, 298 \mathrm{~K}\right): \delta_{\mathrm{H}} 7.63-7.57(\mathrm{~m}$, 2H), 7.45-7.39 (m, 2H), 7.25-7.21 (m, 1H), 7.09-7.04 (m, 2H), 7.03-6.98 (m, 2H) ppm; ${ }^{13} \mathbf{C}$ NMR (125 MHz, $\left.\mathrm{CDCl}_{3}, 298 \mathrm{~K}\right): \delta_{\mathrm{C}} 161.8,154.9,134.3,130.4,125.3,120.5,119.0,118.0$, $106.0 \mathrm{ppm} ; \mathbf{R}_{\mathbf{f}}(9: 1$ hexanes/EtOAc; UV): 0.43.<smiles>CC(C)C1CCC([N+](=O)[O-])CC1Oc1ccc(C#N)cc1</smiles>

4-(((1R,2S,5R)-2-Isopropyl-5-methylcyclohexyl)oxy)benzonitrile (2i) (CAS: 188527-54-0): According to General Procedure A, the product was prepared using the following amounts of reagent: $\mathrm{NiBr}_{2}$ (bpy) $\mathrm{xH}_{2} \mathrm{O}$ (15 mg, $0.040 \mathrm{mmol}, 10 \mathrm{~mol} \%$ ), $\mathrm{Zn}(0)$ dust (52 mg, $0.80 \mathrm{mmol}, 2.0$ equiv), S3 (0.14 g, $0.40 \mathrm{mmol}, 1.0$ equiv), and MPMN (2.0 $\mathrm{mL}$ of a $0.24 \mathrm{M}$ stock solution in DMA, 0.48 mmol, 1.2 equiv). The crude residue was purified by flash column chromatography (gradient of $0-15 \%$ acetone/hexanes) to yield $2 \mathbf{i}$ as a white solid (88 $\mathrm{mg}, 0.342 \mathrm{mmol}, 86 \%$ ). Analytical data: ${ }^{12}{ }^{1} \mathbf{H}$ NMR $\left(400 \mathrm{MHz}, \mathrm{CDCl}_{3}, 298 \mathrm{~K}\right): \delta_{\mathrm{H}} 7.62-7.55(\mathrm{~m}, 2 \mathrm{H}), 6.99-6.91(\mathrm{~m}$, $2 \mathrm{H}), 4.14(\mathrm{td}, J=10.6,4.2 \mathrm{~Hz}, 1 \mathrm{H}), 2.20-2.06(\mathrm{~m}, 2 \mathrm{H}), 1.81-1.71(\mathrm{~m}, 2 \mathrm{H}), 1.63-1.44(\mathrm{~m}, 2 \mathrm{H})$, $1.21-0.89(\mathrm{~m}, 9 \mathrm{H}), 0.77(\mathrm{~d}, J=7.0 \mathrm{~Hz}, 3 \mathrm{H}) \mathrm{ppm} ;{ }^{13} \mathbf{C} \mathbf{N M R}\left(100 \mathrm{MHz}, \mathrm{CDCl}_{3}, 298 \mathrm{~K}\right): \delta_{\mathrm{C}}$ $162.0,134.2,119.5,116.0,103.4,78.0,48.0,40.0,34.5,31.5,26.3,23.9,22.2,20.7,16.8$ ppm; $\mathbf{R}_{\mathbf{f}}\left(9: 1\right.$ hexanes/EtOAc; $\left.\mathrm{UV} / \mathrm{KMnO}_{4}\right): 0.62$.<smiles>COc1ccc(-c2ccc(C#N)cc2)cc1</smiles>

4'-Methoxy-[1,1'-biphenyl]-4-carbonitrile (2h) (CAS: 58743-77-4): According to General Procedure $\mathrm{A}$, the product was prepared using the following amounts of reagent:

$\mathrm{NiBr}_{2}$ (bpy) $x_{2} \mathrm{O}$ (15 mg, $0.040 \mathrm{mmol}, 10 \mathrm{~mol} \%$ ), $\mathrm{Zn}(0)$ dust (52 mg, $0.80 \mathrm{mmol}, 2.0$ equiv), S4 $(0.11 \mathrm{~g}, 0.40 \mathrm{mmol}, 1.0$ equiv), and MPMN (2.0 $\mathrm{mL}$ of a $0.24 \mathrm{M}$ stock solution in DMA, 0.48 mmol, 1.2 equiv). The crude residue was purified by flash column chromatography (gradient of 0-15\% acetone/hexanes) to yield $\mathbf{2 h}$ as a white solid (trial 1: $71 \mathrm{mg}, 0.339 \mathrm{mmol}, 85 \%$; trial 2: $64 \mathrm{mg}, 0.306 \mathrm{mmol}, 77 \%)$. Analytical data: ${ }^{14}{ }^{1} \mathbf{H}$ NMR (500 MHz, $\left.\mathrm{CDCl}_{3}, 298 \mathrm{~K}\right): \delta_{\mathrm{H}} 7.71-7.66$ 
(m, 2H), 7.66-7.61 (m, 2H), 7.56-7.51 (m, 2H), 7.04-6.98 (m, 2H), 3.86 (s, 3H) ppm; ${ }^{13} \mathbf{C}$ NMR $\left(100 \mathrm{MHz}, \mathrm{CDCl}_{3}, 298 \mathrm{~K}\right): \delta_{\mathrm{C}} 160.4,145.4,132.7,131.6,128.5,127.2,119.2,114.7,110.2,55.5$ ppm; $\mathbf{R}_{\mathbf{f}}(9: 1$ hexanes/EtOAc; UV): 0.24.<smiles>N#Cc1ccc(OC(F)(F)F)cc1</smiles>

4-(Trifluoromethoxy)benzonitrile (2j) (CAS: 332-25-2): According to General Procedure A, the product was prepared using the following amounts of reagent: $\mathrm{NiBr}_{2}(\mathrm{bpy}) \bullet x \mathrm{H}_{2} \mathrm{O}(15 \mathrm{mg}$, $0.040 \mathrm{mmol}, 10 \mathrm{~mol} \%$ ), $\mathrm{Zn}(0)$ dust ( $52 \mathrm{mg}, 0.80 \mathrm{mmol}, 2.0$ equiv), 1-bromo-4(trifluoromethoxy)benzene $(59 \mu \mathrm{L}, 0.40 \mathrm{mmol}, 1.0$ equiv), and MPMN (2.0 mL of a $0.24 \mathrm{M}$ stock solution in DMA, $0.48 \mathrm{mmol}, 1.2$ equiv). The crude residue was purified by flash column chromatography (gradient of $0-15 \%$ acetone/hexanes) to yield $\mathbf{2} \mathbf{j}$ as a colourless oil (trial 1: 33 mg, 0.176 mmol, 44\%; trial 2: $33 \mathrm{mg}, 0.176,44 \%)$. Analytical data: ${ }^{13}{ }^{1} \mathbf{H}$ NMR (400 MHz, $\left.\mathrm{CDCl}_{3}, 298 \mathrm{~K}\right): \delta_{\mathrm{H}} 7.75-7.69(\mathrm{~m}, 2 \mathrm{H}), 7.35-7.29(\mathrm{~m}, 2 \mathrm{H}) \mathrm{ppm} ;{ }^{13} \mathbf{C} \mathbf{N M R}\left(100 \mathrm{MHz}, \mathrm{CDCl}_{3}\right.$, $298 \mathrm{~K}): \delta_{\mathrm{C}} 152.4,134.3,121.4,120.3$ (q, J = 258 Hz), 117.8, $111.0 \mathrm{ppm} ;{ }^{19} \mathbf{F}$ NMR $(376 \mathrm{MHz}$, $\left.\mathrm{CDCl}_{3}, 298 \mathrm{~K}\right): \delta_{\mathrm{F}}-57.7 \mathrm{ppm} ; \mathbf{R}_{\mathbf{f}}(9: 1$ hexanes/EtOAc; UV): 0.15.<smiles>COc1cc(C#N)cc(OC)c1</smiles>

3,5-Dimethoxybenzonitrile (2k) (CAS: 19179-31-8): According to General Procedure A, the product was prepared using the following amounts of reagent: $\mathrm{NiBr}_{2}(\mathrm{bpy}) \bullet \mathrm{xH}_{2} \mathrm{O}(15 \mathrm{mg}, 0.040$ mmol, $10 \mathrm{~mol} \%$ ), $\mathrm{Zn}(0)$ dust ( $52 \mathrm{mg}, 0.80 \mathrm{mmol}, 2.0$ equiv), 1-bromo-3,5-dimethoxybenzene ( $87 \mathrm{mg}, 0.40 \mathrm{mmol}, 1.0$ equiv), and MPMN (2.0 mL of a $0.24 \mathrm{M}$ stock solution in DMA, 0.48 mmol, 1.2 equiv). The crude residue was purified by flash column chromatography (gradient of 0-20\% EtOAc/hexanes) to yield $\mathbf{2 k}$ as a colourless oil (trial 1: $63 \mathrm{mg}, 0.386 \mathrm{mmol}, 97 \%$; trial 2: $57 \mathrm{mg}, 0.352 \mathrm{mmol}, 88 \%)$. Analytical data: ${ }^{10}{ }^{1} \mathbf{H}$ NMR $\left(500 \mathrm{MHz}, \mathrm{CDCl}_{3}, 298 \mathrm{~K}\right): \delta_{\mathrm{H}} 6.76$ (d, $J$ $=2.3 \mathrm{~Hz}, 2 \mathrm{H}), 6.65(\mathrm{t}, J=2.3 \mathrm{~Hz}, 1 \mathrm{H}), 3.81(\mathrm{~s}, 6 \mathrm{H}) \mathrm{ppm} ;{ }^{13} \mathbf{C} \mathbf{N M R}\left(125 \mathrm{MHz}, \mathrm{CDCl}_{3}, 298 \mathrm{~K}\right)$ : $\delta_{\mathrm{C}} 161.1,118.9,113.6,110.0,105.8,55.8 \mathrm{ppm} ; \mathbf{R}_{\mathbf{f}}(9: 1$ hexanes/EtOAc): 0.29.<smiles>C=Cc1cccc(C#N)c1</smiles>

3-Vinylbenzonitrile (2ai) (CAS: 5338-96-5): According to General Procedure A, the product was prepared using the following amounts of reagent: $\mathrm{NiBr}_{2}$ (bpy) $\bullet \mathrm{xH}_{2} \mathrm{O}(15 \mathrm{mg}, 0.040 \mathrm{mmol}, 10$ mol \%), $\mathrm{Zn}(0)$ dust (52 mg, $0.80 \mathrm{mmol}, 2.0$ equiv), 3-bromostyrene ( $52 \mu \mathrm{L}, 0.40 \mathrm{mmol}, 1.0$ equiv), and MPMN (2.0 mL of a $0.24 \mathrm{M}$ stock solution in DMA, $0.48 \mathrm{mmol}, 1.2$ equiv). The crude residue was purified by flash column chromatography (gradient of 0-20\% EtOAc/hexanes) to yield 2ai as a colourless oil (trial 1: $12 \mathrm{mg}, 0.093 \mathrm{mmol}, 23 \%$; trial 2: $15 \mathrm{mg}, 0.116 \mathrm{mmol}$, 29\%). Analytical data: ${ }^{15} \mathbf{H}$ NMR (400 MHz, $\left.\mathrm{CDCl}_{3}, 298 \mathrm{~K}\right)$ : $\delta_{\mathrm{H}} 7.72-7.36(\mathrm{~m}, 4 \mathrm{H}), 6.77-6.62$ $(\mathrm{m}, 1 \mathrm{H}), 5.82(\mathrm{dd}, J=17.5,1.8 \mathrm{~Hz}, 1 \mathrm{H}), 5.39(\mathrm{dd}, J=10.9,1.8 \mathrm{~Hz}, 1 \mathrm{H}) \mathrm{ppm} ;{ }^{13} \mathbf{C}$ NMR (100 $\left.\mathrm{MHz}, \mathrm{CDCl}_{3}, 298 \mathrm{~K}\right): \delta_{\mathrm{C}} 138.7,134.8,131.1,130.4,129.8,129.4,118.8,116.6,112.8$ ppm; $\mathbf{R}_{\mathbf{f}}$ (9:1 hexanes/EtOAc; $\left.\mathrm{UV} / \mathrm{KMnO}_{4}\right): 0.47$.<smiles>CC(C)=CCCC(CCOc1ccc(C#N)cc1)[N+](=O)[O-]</smiles> 
4-((3,7-Dimethyloct-6-en-1-yl)oxy)benzonitrile (21): According to General Procedure A, the product was prepared using the following amounts of reagent: $\mathrm{NiBr}_{2}(\mathrm{bpy}) \bullet \mathrm{xH}_{2} \mathrm{O}(15 \mathrm{mg}, 0.040$ mmol, $10 \mathrm{~mol} \%), \mathrm{Zn}(0)$ dust (52 mg, $0.80 \mathrm{mmol}, 2.0$ equiv), $\mathbf{S 5}$ ( $0.12 \mathrm{~g}, 0.40 \mathrm{mmol}, 1.0$ equiv), and MPMN (2.0 mL of a $0.24 \mathrm{M}$ stock solution in DMA, $0.48 \mathrm{mmol}, 1.2$ equiv). The crude residue was purified by flash column chromatography (gradient of $0-15 \%$ acetone/hexanes) to yield $2 \mathrm{l}$ as a colourless oil (trial 1: $88 \mathrm{mg}, 0.342 \mathrm{mmol}, 86 \%$; trial 2: $86 \mathrm{mg}, 0.336 \mathrm{mmol}, 84 \%$ ). Analytical data: ${ }^{12}{ }^{1} \mathbf{H}$ NMR $\left(500 \mathrm{MHz}, \mathrm{CDCl}_{3}, 298 \mathrm{~K}\right): \delta_{\mathrm{H}} 7.60-7.65(\mathrm{~m}, 2 \mathrm{H}), 6.95-6.90(\mathrm{~m}$, 2H), 5.12-5.06 (m, 1H), 4.08-3.99 (m, 2H), 2.09-1.91 (m, 2H), 1.89-1.80 (m, 1H), 1.74-1.56 $(\mathrm{m}, 8 \mathrm{H}), 1.44-1.34(\mathrm{~m}, 1 \mathrm{H}), 1.28-1.19(\mathrm{~m}, 1 \mathrm{H}), 0.95(\mathrm{~d}, J=6.6 \mathrm{~Hz}, 3 \mathrm{H}) \mathrm{ppm} ;{ }^{13} \mathbf{C}$ NMR $(125$ $\left.\mathrm{MHz}_{2} \mathrm{CDCl}_{3}, 298 \mathrm{~K}\right): \delta_{\mathrm{C}} 162.6,134.1,131.6,124.6,119.5,115.3,103.8,66.9,37.2,35.9,29.6$, 25.8, 25.5, 19.6, 17.8 ppm; $\mathbf{R}_{\mathbf{f}}(9: 1$ hexanes/EtOAc; UV/KMnO 4$): 0.47$.<smiles>COc1ccc(C#N)cc1Cl</smiles>

3-Chloro-4-methoxybenzonitrile (2m) (CAS: 102151-33-7): According to General Procedure A, the product was prepared using the following amounts of reagent: $\mathrm{NiBr}_{2}$ (bpy) $\bullet \mathrm{xH}_{2} \mathrm{O}(15 \mathrm{mg}$, $0.040 \mathrm{mmol}, 10 \mathrm{~mol} \%$ ), Zn(0) dust (52 mg, $0.80 \mathrm{mmol}, 2.0$ equiv), S6 ( $89 \mathrm{mg}, 0.40 \mathrm{mmol}, 1.0$ equiv), and MPMN (2.0 $\mathrm{mL}$ of a $0.24 \mathrm{M}$ stock solution in DMA, $0.48 \mathrm{mmol}, 1.2$ equiv). The crude residue was purified by flash column chromatography (gradient of 0-30\% EtOAc/hexanes) to yield $\mathbf{2 m}$ as a white solid (trial 1: $43 \mathrm{mg}, 0.257 \mathrm{mmol}, 64 \%$; trial 2: $45 \mathrm{mg}, 0.268 \mathrm{mmol}, 67 \%$ ). Analytical data: ${ }^{16}{ }^{1} \mathbf{H}$ NMR (400 MHz, $\left.\mathrm{CDCl}_{3}, 298 \mathrm{~K}\right): \delta_{\mathrm{H}} 7.67-7.63(\mathrm{~m}, 1 \mathrm{H}), 7.58-7.52(\mathrm{~m}$, 1H), 7.01-6.95 (m, 1H), 3.96 (s, 3H) ppm; $\left.{ }^{13} \mathbf{C ~ N M R ~ ( 1 0 0 ~ M H z , ~} \mathrm{CDCl}_{3}, 298 \mathrm{~K}\right): \delta_{\mathrm{C}} 158.7$, 133.7, 132.6, 123.7, 118.0, 112.3, 104.9, 56.6 ppm; $\mathbf{R}_{\mathbf{f}}(9: 1$ hexanes/EtOAc; UV): 0.12.<smiles>COc1ccc(C#N)cc1F</smiles>

3-Fluoro-4-methoxybenzonitrile (2n) (CAS: 331-62-4): According to General Procedure A, the product was prepared using the following amounts of reagent: $\mathrm{NiBr}_{2}$ (bpy) $\bullet \mathrm{xH}_{2} \mathrm{O}(15 \mathrm{mg}$, $0.040 \mathrm{mmol}, 10 \mathrm{~mol} \mathrm{\%}), \mathrm{Zn}(0)$ dust (52 mg, $0.80 \mathrm{mmol}, 2.0$ equiv), 3-fluoro-4methoxybenzonitrile (52 $\mu \mathrm{L}, 0.40 \mathrm{mmol}, 1.0$ equiv), and MPMN (2.0 mL of a $0.24 \mathrm{M}$ stock solution in DMA, 0.48 mmol, 1.2 equiv). The crude residue was purified by flash column chromatography (gradient of 5-30\% acetone/hexanes) to yield $2 \mathrm{n}$ as a white solid (trial 1: $41 \mathrm{mg}$, $0.271 \mathrm{mmol}, 68 \%$; trial 2: $44 \mathrm{mg}, 0.291 \mathrm{mmol}, 73 \%)$. Analytical data: ${ }^{17}{ }^{1} \mathbf{H}$ NMR (400 MHz, $\left.\mathrm{CDCl}_{3}, 298 \mathrm{~K}\right): \delta_{\mathrm{H}} 7.46-7.40(\mathrm{~m}, 1 \mathrm{H}), 7.38-7.32(\mathrm{~m}, 1 \mathrm{H}), 7.01(\mathrm{t}, J=8.4 \mathrm{~Hz}, 1 \mathrm{H}), 3.95(\mathrm{~s}, 3 \mathrm{H})$ ppm; ${ }^{13}$ C NMR $\left(100 \mathrm{MHz}, \mathrm{CDCl}_{3}, 298 \mathrm{~K}\right): \delta_{\mathrm{C}} 152.0(\mathrm{~d}, J=10.2 \mathrm{~Hz}) 151.9(\mathrm{~d}, J=248.7 \mathrm{~Hz})$, $129.8(\mathrm{~d}, J=4.0 \mathrm{~Hz}), 119.7(\mathrm{~d}, J=21.1 \mathrm{~Hz}), 118.1(\mathrm{~d}, J=2.6 \mathrm{~Hz}), 113.7(\mathrm{~d}, J=2.5 \mathrm{~Hz}), 104.1$ $(\mathrm{d}, J=8.1 \mathrm{~Hz}), 56.5 \mathrm{ppm} ;{ }^{19} \mathbf{F}$ NMR $\left(376 \mathrm{MHz}, \mathrm{CDCl}_{3}, 298 \mathrm{~K}\right): \delta_{\mathrm{F}}-131.9 \mathrm{ppm} ; \mathbf{R}_{\mathbf{f}}(9: 1$ hexanes/EtOAc; UV): 0.18 .

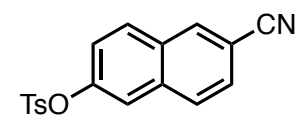

6-Cyanonaphthalen-2-yl 4-methylbenzenesulfonate (2aj) (CAS: 939822-86-3): According to General Procedure A, the product was prepared using the following amounts of reagent: $\mathrm{NiBr}_{2}$ (bpy) $\bullet \mathrm{xH}_{2} \mathrm{O}$ (15 mg, $0.040 \mathrm{mmol}, 10 \mathrm{~mol} \%$ ), Zn(0) dust (52 mg, $0.80 \mathrm{mmol}, 2.0$ equiv), S7 (0.15 g, $0.40 \mathrm{mmol}, 1.0$ equiv), and MPMN (2.0 mL of a $0.24 \mathrm{M}$ stock solution in DMA, 0.48 
mmol, 1.2 equiv). The crude residue was purified by flash column chromatography (gradient of 0-30\% EtOAc/hexanes) to yield 2aj as a white solid (trial 1: $56 \mathrm{mg}, 0.173 \mathrm{mmol}, 43 \%$; trial 2: $60 \mathrm{mg}, 0.184 \mathrm{mmol}, 46 \%)$. Analytical data: ${ }^{18}{ }^{1} \mathrm{H} \mathrm{NMR}\left(400 \mathrm{MHz}, \mathrm{CDCl}_{3}, 298 \mathrm{~K}\right): \delta_{\mathrm{H}} 8.24-8.17$ $(\mathrm{m}, 1 \mathrm{H}), 7.88-7.80(\mathrm{~m}, 2 \mathrm{H}), 7.78-7.70(\mathrm{~m}, 2 \mathrm{H}), 7.66-7.60(\mathrm{~m}, 1 \mathrm{H}), 7.59-7.55(\mathrm{~m}, 1 \mathrm{H}), 7.36-$ $7.29(\mathrm{~m}, 2 \mathrm{H}), 7.27-7.18(\mathrm{~m}, 1 \mathrm{H}), 2.46(\mathrm{~s}, 3 \mathrm{H}) \mathrm{ppm} ;{ }^{13} \mathbf{C} \mathbf{N M R}\left(100 \mathrm{MHz}, \mathrm{CDCl}_{3}, 298 \mathrm{~K}\right): \delta_{\mathrm{C}}$ 149.5, 145.9, 135.1, 134.0, 132.3, 130.8, 130.6, 130.1, 129.3, 128.6, 127.5, 123.4, 120.3, 118.9, 110.1, 21.9 ppm; $\mathbf{R}_{\mathbf{f}}(9: 1$ hexanes/EtOAc; UV): 0.12 .<smiles>N#Cc1cccc(C2OCCO2)c1</smiles>

3-(1,3-dioxolan-2-yl)benzonitrile (2o) (CAS: 153329-04-5): According to General Procedure A, the product was prepared using the following amounts of reagent: $\mathrm{NiBr}_{2}$ (bpy) $\bullet \mathrm{xH}_{2} \mathrm{O}(15 \mathrm{mg}$, $0.040 \mathrm{mmol}, 10 \mathrm{~mol} \%), \mathrm{Zn}(0)$ dust (52 mg, $0.80 \mathrm{mmol}, 2.0$ equiv), $\mathbf{S 8}$ (61 $\mu \mathrm{L}, 0.40 \mathrm{mmol}, 1.0$ equiv), and MPMN (2.0 mL of a $0.24 \mathrm{M}$ stock solution in DMA, $0.48 \mathrm{mmol}, 1.2$ equiv). The crude residue was purified by flash column chromatography (gradient of 0-30\% EtOAc/hexanes) to yield 20 as a colourless oil (trial 1: $33 \mathrm{mg}, 0.188 \mathrm{mmol}, 47 \%$; trial 2: $39 \mathrm{mg}, 0.223 \mathrm{mmol}$, 56\%). Analytical data: ${ }^{19}{ }^{1} \mathbf{H}$ NMR $\left(500 \mathrm{MHz}, \mathrm{CDCl}_{3}, 298 \mathrm{~K}\right): \delta_{\mathrm{H}} 7.81-7.77(\mathrm{~m}, 1 \mathrm{H}), 7.73-7.68$ (m, 1H), 7.77-7.62 (m, 1H), 7.52-7.46 (m, 1H), $5.82(\mathrm{~s}, 1 \mathrm{H}), 4.17-4.00(\mathrm{~m}, 4 \mathrm{H}) \mathrm{ppm} ;{ }^{13} \mathbf{C}$ NMR (125 MHz, $\left.\mathrm{CDCl}_{3}, 298 \mathrm{~K}\right): \delta_{\mathrm{C}} 139.8,132.8,131.1,130.3,129.3,118.7,112.6,102.4,65.6 \mathrm{ppm}$; $\mathbf{R}_{\mathbf{f}}\left(9: 1\right.$ hexanes/EtOAc; $\left.\mathrm{UV} / \mathrm{KMnO}_{4}\right): 0.15$.

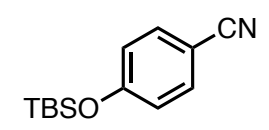

4-((tert-Butyldimethylsilyl)oxy)benzonitrile (2ak) (CAS: 117635-43-5): According to General Procedure $\mathrm{A}$, the product was prepared using the following amounts of reagent:

$\mathrm{NiBr}_{2}$ (bpy) $\mathrm{xH}_{2} \mathrm{O}$ (15 mg, $0.040 \mathrm{mmol}, 10 \mathrm{~mol} \%$ ), Zn(0) dust (52 mg, $0.80 \mathrm{mmol}, 2.0$ equiv), S9 $(0.11 \mathrm{~g}, 0.40 \mathrm{mmol}, 1.0$ equiv), and MPMN (2.0 $\mathrm{mL}$ of a $0.24 \mathrm{M}$ stock solution in DMA, 0.48 mmol, 1.2 equiv). The crude residue was purified by flash column chromatography (hexanes) to yield 2ak as a colourless oil (trial 1: $13 \mathrm{mg}, 0.056 \mathrm{mmol}, 14 \%$; trial 2: $31 \mathrm{mg}, 0.132 \mathrm{mmol}$, 33\%). Analytical data: ${ }^{20} \mathbf{H}$ NMR (400 MHz, $\left.\mathrm{CDCl}_{3}, 298 \mathrm{~K}\right): \delta_{\mathrm{H}} 7.57-7.50(\mathrm{~m}, 2 \mathrm{H}), 6.92-6.85$ (m, 2H), 0.98 (s, 9H), 0.23 (s, 6H) ppm; $\left.{ }^{13} \mathbf{C ~ N M R ~ ( 1 0 0 ~ M H z , ~} \mathrm{CDCl}_{3}, 298 \mathrm{~K}\right): \delta_{\mathrm{C}} 159.8,134.2$, 121.0, 119.4, 104.8, 25.7, 18.4, -4.3 ppm; $\mathbf{R}_{\mathbf{f}}(9: 1$ hexanes/EtOAc; UV): 0.56.<smiles>N#Cc1ccc(C#N)c(N)c1</smiles>

2-Methylterephthalonitrile (2p) (CAS: 55984-93-5): According to General Procedure A, the product was prepared using the following amounts of reagent: $\mathrm{NiBr}_{2}(\mathrm{bpy}) \cdot \mathrm{xH}_{2} \mathrm{O}(15 \mathrm{mg}, 0.040$ mmol, $20 \mathrm{~mol} \%), \mathrm{Zn}(0)$ dust (52 mg, $0.80 \mathrm{mmol}, 4.0$ equiv), 1,4-dibromo-2-methylbenzene (51 $\mu \mathrm{L}, 0.20 \mathrm{mmol}, 1.0$ equiv), and MPMN (2.0 mL of a $0.24 \mathrm{M}$ stock solution in DMA, $0.48 \mathrm{mmol}$, 2.4 equiv). The crude residue was purified by flash column chromatography (gradient of 0-30\% EtOAc/hexanes) to yield 2p as a white solid (trial 1: $20 \mathrm{mg}, 0.141,71 \%$; trial 2: $22 \mathrm{mg}, 0.156$ mmol, 78\%). Analytical data: ${ }^{21}{ }^{1} \mathbf{H}$ NMR $\left(500 \mathrm{MHz}, \mathrm{CDCl}_{3}, 298 \mathrm{~K}\right): \delta_{\mathrm{H}} 7.72(\mathrm{~d}, J=8.0 \mathrm{~Hz}, 1 \mathrm{H})$, 7.65-7.62 (m, 1H), 7.60-7.56 (m, 1H), 2.61 (s, 3H) ppm; ${ }^{13} \mathbf{C ~ N M R ~ ( 1 2 5 ~ M H z , ~ C D C l ~} 3$, $\left.298 \mathrm{~K}\right)$ : 
$\delta_{\mathrm{C}} 143.4,133.8,133.2,129.9,117.38,117.35,116.6,116.5,20.6 \mathrm{ppm} ; \mathbf{R}_{\mathbf{f}}(9: 1$ hexanes/EtOAc; $\left.\mathrm{UV} / \mathrm{KMnO}_{4}\right): 0.22$.<smiles>N#Cc1ccc(-c2ccc(C#N)cc2)cc1</smiles>

[1,1'-Biphenyl]-4,4'-dicarbonitrile (2q) (CAS: 1591-30-6): According to General Procedure A, the product was prepared using the following amounts of reagent: $\mathrm{NiBr}_{2}(\mathrm{bpy}) \bullet \mathrm{xH}_{2} \mathrm{O}(15 \mathrm{mg}$, $0.040 \mathrm{mmol}, 20 \mathrm{~mol} \%$ ), $\mathrm{Zn}(0)$ dust ( $52 \mathrm{mg}, 0.80 \mathrm{mmol}, 4.0$ equiv), 4,4'-dibromobiphenyl (62 $\mathrm{mg}, 0.20 \mathrm{mmol}, 1.0$ equiv), and MPMN (2.0 mL of a $0.24 \mathrm{M}$ stock solution in DMA, $0.48 \mathrm{mmol}$, 2.4 equiv). The crude residue was purified by flash column chromatography (gradient of $0-40 \%$ EtOAc/hexanes) to yield 2q as a white solid (trial $1: 27 \mathrm{mg}, 0.132 \mathrm{mmol}, 66 \%$; trial 2: $23 \mathrm{mg}$, $0.114 \mathrm{mmol}, 57 \%)$. Analytical data: ${ }^{22} \mathbf{H}$ NMR $\left(500 \mathrm{MHz}, \mathrm{CDCl}_{3}, 298 \mathrm{~K}\right): \delta_{\mathrm{H}} 7.81-7.76(\mathrm{~m}$, 4H), 7.72-7.66 (m, 4H) ppm; ${ }^{13} \mathbf{C}$ NMR (125 MHz, $\left.\mathrm{CDCl}_{3}, 298 \mathrm{~K}\right): \delta_{\mathrm{C}} 143.7,133.0,128.1$, 118.1, $112.6 \mathrm{ppm} ; \mathbf{R}_{\mathbf{f}}(9: 1$ hexanes/EtOAc; UV): 0.14 .<smiles>N#Cc1ccc(-n2cccn2)cc1</smiles>

4-(1H-Pyrazol-1-yl)benzonitrile (2aa) (CAS: 25699-83-6): According to General Procedure A, the product was prepared using the following amounts of reagent: $\mathrm{NiBr}_{2}(\mathrm{bpy}) \bullet \mathrm{xH}_{2} \mathrm{O}(15 \mathrm{mg}$, $0.040 \mathrm{mmol}, 10 \mathrm{~mol} \%$ ), Zn(0) dust (52 mg, $0.80 \mathrm{mmol}, 2.0$ equiv), $\mathbf{S 1 0}(89 \mathrm{mg}, 0.40 \mathrm{mmol}, 1.0$ equiv), and MPMN (2.0 mL of a $0.24 \mathrm{M}$ stock solution in DMA, $0.48 \mathrm{mmol}, 1.2$ equiv). The crude residue was purified by flash column chromatography (gradient of 0-50\% EtOAc/hexanes) to yield 2aa as an off-white solid (trial 1: $47 \mathrm{mg}, 0.280 \mathrm{mmol}, 70 \%$; trial 2: $53 \mathrm{mg}, 0.316 \mathrm{mmol}$, 79\%). Analytical data: ${ }^{10}{ }^{1} \mathbf{H}$ NMR $\left(400 \mathrm{MHz}, \mathrm{CDCl}_{3}, 298 \mathrm{~K}\right): \delta_{\mathrm{H}} 7.99$ (d, $\left.J=2.6 \mathrm{~Hz}, 1 \mathrm{H}\right), 7.87-$ $7.82(\mathrm{~m}, 2 \mathrm{H}), 7.80-7.73(\mathrm{~m}, 3 \mathrm{H}), 6.54(\mathrm{dd}, J=2.6,1.7 \mathrm{~Hz}, 1 \mathrm{H}) \mathrm{ppm} ;{ }^{13} \mathbf{C}$ NMR $(100 \mathrm{MHz}$, $\left.\mathrm{CDCl}_{3}, 298 \mathrm{~K}\right): \delta_{\mathrm{C}} 142.8,142.6,133.8,126.9,119.1,118.5,109.8,109.2 \mathrm{ppm} ; \mathrm{R}_{\mathrm{f}}(9: 1$ EtOAc/hexanes; UV): 0.31 .<smiles>N#Cc1ccc([Nb])cc1</smiles>

4-(Dibenzylamino)benzonitrile (2r) (CAS: 52805-39-7): According to General Procedure A, the product was prepared using the following amounts of reagent: $\mathrm{NiBr}_{2}(\mathrm{bpy}) \bullet \mathrm{xH}_{2} \mathrm{O}(15 \mathrm{mg}$, $0.040 \mathrm{mmol}, 10 \mathrm{~mol} \%$ ), $\mathrm{Zn}(0)$ dust (52 mg, $0.80 \mathrm{mmol}, 2.0$ equiv), S11 (0.14 g, $0.40 \mathrm{mmol}, 1.0$ equiv), and MPMN ( $2.0 \mathrm{~mL}$ of a $0.24 \mathrm{M}$ stock solution in DMA, $0.48 \mathrm{mmol}, 1.2$ equiv). The crude residue was purified by flash column chromatography (gradient of $0-30 \%$ EtOAc/hexanes) to yield $2 \mathbf{r}$ as a white solid $(0.10 \mathrm{~g}, 0.335 \mathrm{mmol}, 84 \%)$. Analytical data: ${ }^{23}{ }^{1} \mathbf{H}$ NMR $(400 \mathrm{MHz}$, $\left.\mathrm{CDCl}_{3}, 298 \mathrm{~K}\right): \delta_{\mathrm{H}} 7.44-7.27(\mathrm{~m}, 8 \mathrm{H}), 7.25-7.19(\mathrm{~m}, 4 \mathrm{H}), 6.76-6.70(\mathrm{~m}, 2 \mathrm{H}), 4.73(\mathrm{~s}, 4 \mathrm{H}) \mathrm{ppm}$; ${ }^{13} \mathrm{C}$ NMR $\left(100 \mathrm{MHz}, \mathrm{CDCl}_{3}, 298 \mathrm{~K}\right): \delta_{\mathrm{C}} 152.0,136.9,133.8,129.1,127.6,126.5,120.5,112.2$, 98.9, 54.3 ppm; $\mathbf{R}_{\mathbf{f}}(9: 1$ hexanes/EtOAc; UV/KMnO 4$): 0.19$.<smiles>N#Cc1cccc(N)c1</smiles> 
Isophthalonitrile (2ai) (CAS: 626-17-5): According to General Procedure A, the product was prepared using the following amounts of reagent: $\mathrm{NiBr}_{2}$ (bpy) $\bullet \mathrm{xH}_{2} \mathrm{O}(15 \mathrm{mg}, 0.040 \mathrm{mmol}, 10$ mol \%), Zn(0) dust (52 mg, $0.80 \mathrm{mmol}, 2.0$ equiv), 3-bromobenzonitrile ( $73 \mathrm{mg}, 0.40 \mathrm{mmol}, 1.0$ equiv), and MPMN (2.0 mL of a $0.24 \mathrm{M}$ stock solution in DMA, $0.48 \mathrm{mmol}, 1.2$ equiv). The crude residue was purified by flash column chromatography (gradient of 0-20\% EtOAc/hexanes) to yield 2ai as an off-white solid (trial 1: $29 \mathrm{mg}, 0.226 \mathrm{mmol}, 57 \%$; trial 2: $25 \mathrm{mg}, 0.200 \mathrm{mmol}$, 50\%). Analytical data: ${ }^{24}{ }^{1}$ H NMR 7.98-7.94 (m, 1H), 7.94-7.87 (m, 2H), 7.69-7.62 (m, 1H) ppm; ${ }^{13} \mathbf{C}$ NMR (100 MHz, $\left.\mathrm{CDCl}_{3}, 298 \mathrm{~K}\right): \delta_{\mathrm{H}} 136.1,135.6,130.5,116.7,114.4$ ppm; $\mathbf{R}_{\mathbf{f}}(9: 1)$ : 0.15 .<smiles></smiles>

4-(1-Hydroxyethyl)benzonitrile (2s) (CAS: 52067-35-3): According to General Procedure A, the product was prepared using the following amounts of reagent: $\mathrm{NiBr}_{2}$ (bpy) $\bullet \mathrm{xH}_{2} \mathrm{O}(15 \mathrm{mg}$, $0.040 \mathrm{mmol}, 10 \mathrm{~mol} \%$ ), Zn(0) dust (52 mg, $0.80 \mathrm{mmol}, 2.0$ equiv), S12 (55 $\mu \mathrm{L}, 0.40 \mathrm{mmol}, 1.0$ equiv), and MPMN (2.0 mL of a $0.24 \mathrm{M}$ stock solution in DMA, $0.48 \mathrm{mmol}, 1.2$ equiv). The crude residue was purified by flash column chromatography (gradient of 0-20\% EtOAc/hexanes) to yield $2 \mathrm{~s}$ as a colourless oil (trial 1: $37 \mathrm{mg}, 0.251 \mathrm{mmol}, 65 \%$; trial 2: $38 \mathrm{mg}, 0.256 \mathrm{mmol}$, 64\%). Analytical data: ${ }^{25}{ }^{1} \mathbf{H}$ NMR (400 MHz, $\left.\mathrm{CDCl}_{3}, 298 \mathrm{~K}\right): \delta_{\mathrm{H}} 7.67-7.57(\mathrm{~m}, 2 \mathrm{H}), 7.52-7.42$ $(\mathrm{m}, 2 \mathrm{H}), 4.94$ (br q, $J=6.6 \mathrm{~Hz}, 1 \mathrm{H}), 2.29$ (br s, 1H), 1.56-1.43 (m, 3H) ppm; ${ }^{13} \mathbf{C}$ NMR (100 $\left.\mathrm{MHz}, \mathrm{CDCl}_{3}, 298 \mathrm{~K}\right): \delta_{\mathrm{C}} 151.2,132.3,126.1,118.9,115.7,69.6,25.4$ ppm; $\mathbf{R}_{\mathbf{f}}(9: 1$ hexanes/EtOAc; $\left.\mathrm{UV} / \mathrm{KMnO}_{4}\right)$ : 0.06.<smiles>CCOC(=O)c1ccc(C#N)cc1</smiles>

Ethyl 4-cyanobenzoate (2t) (CAS: 7153-22-2): According to General Procedure A, the product was prepared using the following amounts of reagent: $\mathrm{NiBr}_{2}$ (bpy) $\bullet \mathrm{xH}_{2} \mathrm{O}(15 \mathrm{mg}, 0.040 \mathrm{mmol}, 10$ mol \%), $\mathrm{Zn}(0)$ dust (52 mg, $0.80 \mathrm{mmol}, 2.0$ equiv), ethyl 4-bromobenzoate ( $65 \mu \mathrm{L}, 0.40 \mathrm{mmol}$, 1.0 equiv), and MPMN (2.0 mL of a $0.24 \mathrm{M}$ stock solution in DMA, $0.48 \mathrm{mmol}, 1.2$ equiv). The crude residue was purified by flash column chromatography (gradient of $100 \%$ hexanes to $80 \%$ $\mathrm{PhMe} /$ hexanes to 20\% EtOAc/hexanes) to yield $\mathbf{2 t}$ as a white solid (trial 1: $69 \mathrm{mg}, 0.394 \mathrm{mmol}$, 99\%; trial 2: $69 \mathrm{mg}, 0.394 \mathrm{mmol}, 99 \%)$. Analytical data: ${ }^{26}{ }^{1} \mathbf{H} \mathbf{N M R}\left(500 \mathrm{MHz}, \mathrm{CDCl}_{3}, 298 \mathrm{~K}\right)$ : $\delta_{\mathrm{H}} 8.16-8.11(\mathrm{~m}, 2 \mathrm{H}), 7.71-7.66(\mathrm{~m}, 2 \mathrm{H}), 4.41(\mathrm{q}, J=7.2 \mathrm{~Hz}, 2 \mathrm{H}), 1.42(\mathrm{t}, J=7.1 \mathrm{~Hz}, 3 \mathrm{H}) \mathrm{ppm}$; ${ }^{13} \mathbf{C}$ NMR $\left(125 \mathrm{MHz}, \mathrm{CDCl}_{3}, 298 \mathrm{~K}\right): \delta_{\mathrm{C}} 166.5,144.5,130.3,130.2,127.4,61.3,14.5 \mathrm{ppm} ; \mathbf{R}_{\mathbf{f}}$ (9:1 hexanes/EtOAc; UV): 0.22.<smiles>N#Cc1ccc2c(c1)COC2=O</smiles>

1-Oxo-1,3-dihydroisobenzofuran-5-carbonitrile (2am) (CAS: 82104-74-3): According to General Procedure A, the product was prepared using the following amounts of reagent: $\mathrm{NiBr}_{2}$ (bpy) $\bullet \mathrm{xH}_{2} \mathrm{O}$ (15 mg, $0.040 \mathrm{mmol}, 10 \mathrm{~mol} \%$ ), $\mathrm{Zn}(0)$ dust (52 mg, $0.80 \mathrm{mmol}, 2.0$ equiv), 5 bromophthalide ( $85 \mathrm{mg}, 0.40 \mathrm{mmol}, 1.0$ equiv), and MPMN (2.0 mL of a $0.24 \mathrm{M}$ stock solution in DMA, $0.48 \mathrm{mmol}, 1.2$ equiv). The crude residue was purified by flash column 
chromatography (gradient of 30-70\% EtOAc/hexanes) to yield 2am as a white solid (trial 1: 13 $\mathrm{mg}, 0.082 \mathrm{mmol}, 21 \%$; trial 2: $36 \mathrm{mg}, 0.226 \mathrm{mmol}, 57 \%)$. Analytical data: ${ }^{27}{ }^{1} \mathbf{H}$ NMR (400 $\left.\mathrm{MHz}, \mathrm{CDCl}_{3}, 298 \mathrm{~K}\right): \delta_{\mathrm{H}} 8.09-8.03(\mathrm{~m}, 1 \mathrm{H}), 7.88-7.81(\mathrm{~m}, 2 \mathrm{H}), 5.40$ (br s, $\left.2 \mathrm{H}\right) \mathrm{ppm} ;{ }^{13} \mathbf{C}$ NMR $\left(100 \mathrm{MHz}, \mathrm{CDCl}_{3}, 298 \mathrm{~K}\right): \delta_{\mathrm{C}} 169.1,146.8,133.0,129.7,126.9,126.5,117.8,117.6,69.4 \mathrm{ppm}$; $\mathbf{R}_{\mathbf{f}}(80 \%$ EtOAc/hexanes; UV): 0.46.<smiles>N#Cc1ccc(C(=O)Nc2ccccc2)cc1</smiles>

4-Cyano-N,N-dimethylbenzamide (2u) (CAS: 24167-50-8): According to General Procedure A, the product was prepared using the following amounts of reagent: $\mathrm{NiBr}_{2}(\mathrm{bpy}) \cdot \mathrm{xH}_{2} \mathrm{O}(15 \mathrm{mg}$, $0.040 \mathrm{mmol}, 10 \mathrm{~mol} \%$ ), $\mathrm{Zn}(0)$ dust (52 mg, $0.80 \mathrm{mmol}, 2.0$ equiv), $\mathbf{S 1 3}$ (91 mg, $0.40 \mathrm{mmol}, 1.0$ equiv), and MPMN (2.0 mL of a $0.24 \mathrm{M}$ stock solution in DMA, $0.48 \mathrm{mmol}, 1.2$ equiv). The crude residue was purified by flash column chromatograph (gradient of 0-50\% EtOAc/hexanes) to yield $2 \mathbf{u}$ as an off-white solid (trial 1: $99 \mathrm{mg}, 0.228 \mathrm{mmol}, 57 \%$; trial 2: $99 \mathrm{mg}, 0.228 \mathrm{mmol}$, 57\%). Analytical data: ${ }^{28}{ }^{1} \mathbf{H}$ NMR $\left(400 \mathrm{MHz}, \mathrm{CDCl}_{3}, 298 \mathrm{~K}\right): \delta_{\mathrm{H}} 7.74-7.68(\mathrm{~m}, 2 \mathrm{H}), 7.55-7.49$ (m, 2H), 3.12 (s, 3H), 2.95 (s, 3H) ppm; ${ }^{13} \mathbf{C}$ NMR (100 MHz, CDCl, $\left.298 \mathrm{~K}\right): \delta_{\mathrm{C}} 169.7,140.8$, $132.5,127.9,118.3,113.5,39.5,35.5$ ppm; $\mathbf{R}_{\mathbf{f}}(80 \%$ EtOAc/hexanes; UV): 0.46.

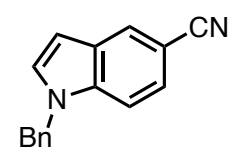

1-benzyl-1H-indole-5-carbonitrile (2v) (CAS: 80531-13-1): According to General Procedure A, the product was prepared using the following amounts of reagent: $\mathrm{NiBr}_{2}(\mathrm{bpy}) \cdot \mathrm{xH}_{2} \mathrm{O}(15 \mathrm{mg}$, $0.040 \mathrm{mmol}, 10 \mathrm{~mol} \%$ ), $\mathrm{Zn}(0)$ dust (52 mg, $0.80 \mathrm{mmol}, 2.0$ equiv), $\mathbf{S 1 2}$ (0.11 g, $0.40 \mathrm{mmol}, 1.0$ equiv), and MPMN (2.0 mL of a $0.24 \mathrm{M}$ stock solution in DMA, $0.48 \mathrm{mmol}, 1.2$ equiv). The crude residue was purified by flash column chromatography (gradient of 0-20\% EtOAc/hexanes) to yield $2 \mathrm{v}$ as a colourless oil (trial 1: $76 \mathrm{mg}, 0.327 \mathrm{mmol}, 82 \%$; trial 2: $72 \mathrm{mg}, 0.308 \mathrm{mmol}$, 77\%). Analytical data: ${ }^{29}{ }^{1} \mathbf{H}$ NMR $\left(400 \mathrm{MHz}, \mathrm{CDCl}_{3}, 298 \mathrm{~K}\right): \delta_{\mathrm{H}} 8.01-7.96(\mathrm{~m}, 1 \mathrm{H}), 7.44-7.36$ (m, 1H), 7.35-7.23 (m, 5H), 7.12-7.06 (m, 2H), 6.66-6.61 (m, 1H), 5.35 (s, 2H) ppm; ${ }^{13} \mathbf{C}$ NMR $\left(100 \mathrm{MHz}, \mathrm{CDCl}_{3}, 298 \mathrm{~K}\right): \delta_{\mathrm{C}} 137.9,136.5,130.7,129.1,128.6,128.2,126.9,126.7,124.8$, 120.9, 110.7, 102.9, 102.9, 50.5 ppm; $\mathbf{R}_{\mathbf{f}}(9: 1$ hexanes/EtOAc; UV): 0.21 .<smiles></smiles>

9-(tert-Butyldimethylsilyl)-9H-carbazole-2-carbonitrile (2w): According to General Procedure A, the product was prepared using the following amounts of reagent: $\mathrm{NiBr}_{2}(\mathrm{bpy}) \cdot \mathrm{xH}_{2} \mathrm{O}(15 \mathrm{mg}, 0.040 \mathrm{mmol}, 10 \mathrm{~mol} \mathrm{\%}), \mathrm{Zn}(0)$ dust (52 mg, $0.80 \mathrm{mmol}, 2.0$ equiv), S13 (0.14 g, $0.40 \mathrm{mmol}, 1.0$ equiv), and MPMN ( $2.0 \mathrm{~mL}$ of a $0.24 \mathrm{M}$ stock solution in DMA, $0.48 \mathrm{mmol}, 1.2$ equiv). The crude residue was purified by flash column chromatography (gradient of 0-20\% EtOAc/hexanes) to yield $\mathbf{2 w}$ as a white solid (trial 1: $80 \mathrm{mg}, 0.261 \mathrm{mmol}$, 65\%; trial 2: $74 \mathrm{mg}, 0.240 \mathrm{mmol}, 60 \%)$. Analytical data: ${ }^{1} \mathbf{H}$ NMR $\left(400 \mathrm{MHz}, \mathrm{CDCl}_{3}, 298 \mathrm{~K}\right): \delta_{\mathrm{H}}$ 8.14-8.07 (m, 2H), 7.89-7.87 (m, 1H), 7.67-7.62 (m, 1H), 7.52-7.43 (m, 2H), 7.32-7.27 (m, $1 \mathrm{H}), 1.04$ (s, 9H), 0.78 (s, 6H) ppm; ${ }^{13} \mathbf{C}$ NMR $\left(100 \mathrm{MHz}, \mathrm{CDCl}_{3}, 298 \mathrm{~K}\right): \delta_{\mathrm{C}} 146.4,144.1$, $130.1,127.4,125.2,123.0,120.9,120.7,120.6,120.6,118.1,114.6,107.8,26.6,20.6,-1.1 \mathrm{ppm}$; 
HRMS $\boldsymbol{m} / \boldsymbol{z}$ (DART): calcd for $\mathrm{C}_{19} \mathrm{H}_{23} \mathrm{~N}_{2} \mathrm{Si}(\mathrm{M}+\mathrm{H}): 307.1631$; 307.1612; IR (neat): 3049, 2983, 2958, 2931, 2885, 2858, 2214, 1625, 1470, 1454, 1422, 1271, 1254, 1156, 1118, 822, 718, 690, $630 \mathrm{~cm}^{-1}$; m.p.: $106-108^{\circ} \mathrm{C} ; \mathrm{R}_{\mathrm{f}}(7: 3$ hexanes/PhMe; UV): 0.30 .<smiles>N#Cc1ccc2oc3ccccc3c2c1</smiles>

Dibenzo[b,d]furan-2-carbonitrile (2x) (CAS: 20927-96-2): According to General Procedure A, the product was prepared using the following amounts of reagent: $\mathrm{NiBr}_{2}(\mathrm{bpy}) \cdot \mathrm{xH}_{2} \mathrm{O}(15 \mathrm{mg}$, $0.040 \mathrm{mmol}, 10 \mathrm{~mol} \%$ ), $\mathrm{Zn}(0)$ dust (52 mg, $0.80 \mathrm{mmol}, 2.0$ equiv), S14 (99 mg, $0.40 \mathrm{mmol}, 1.0$ equiv), and MPMN (2.0 mL of a $0.24 \mathrm{M}$ stock solution in DMA, $0.48 \mathrm{mmol}, 1.2$ equiv). The crude residue was purified by flash column chromatography (gradient of 0-15\% EtOAc/hexanes) to yield $\mathbf{2 x}$ as a white solid, which sample contained a small amount (ca. 10\%) of dibenzofuran (trial 1: $52 \mathrm{mg}, 0.269 \mathrm{mmol}, 82 \%$; trial 2: $70 \mathrm{mg}, 0.360 \mathrm{mmol}, 90 \%$ ). Analytical data: ${ }^{30}{ }^{1} \mathbf{H}$ NMR $\left(400 \mathrm{MHz}, \mathrm{CDCl}_{3}, 298 \mathrm{~K}\right): \delta_{\mathrm{H}} 8.29-8.24(\mathrm{~m}, 1 \mathrm{H}), 8.00-7.93(\mathrm{~m}, 1 \mathrm{H}), 7.77-7.70(\mathrm{~m}, 1 \mathrm{H}), 7.67-$ 7.59 (m, 2H), 7.59-7.51 (m, 1H), 7.46-7.39 (m, 1H) ppm; ${ }^{13} \mathbf{C}$ NMR $\left(100 \mathrm{MHz} \mathrm{CDCl}_{3}, 298 \mathrm{~K}\right)$ : $\delta_{\mathrm{C}} 158.1,156.9,131.0,128.9,125.5,125.4,123.9,122.7,121.2,119.3,113.0,112.2,106.7 \mathrm{ppm}$; $\mathbf{R}_{\mathbf{f}}\left(9: 1\right.$ hexanes/EtOAc; $\left.\mathrm{UV} / \mathrm{KMnO}_{4}\right): 0.53$.<smiles>N#Cc1ccc2sccc2c1</smiles>

Benzo[b]thiophene-5-carbonitrile (2y) (CAS: 2060-63-1): According to General Procedure A, the product was prepared using the following amounts of reagent: $\mathrm{NiBr}_{2}(\mathrm{bpy}) \cdot \mathrm{xH}_{2} \mathrm{O}(15 \mathrm{mg}$, $0.040 \mathrm{mmol}, 10 \mathrm{~mol} \%$ ), $\mathrm{Zn}(0)$ dust ( $52 \mathrm{mg}, 0.80 \mathrm{mmol}, 2.0$ equiv), 5-bromobenzothiophene ( 85 $\mathrm{mg}, 0.40 \mathrm{mmol}, 1.0$ equiv), and MPMN (2.0 mL of a $0.24 \mathrm{M}$ stock solution in DMA, $0.48 \mathrm{mmol}$, 1.2 equiv). The crude residue was purified by flash column chromatography (gradient of $0-15 \%$ EtOAc/hexanes) to yield $\mathbf{2 y}$ as a white solid $(45 \mathrm{mg}, 0.283 \mathrm{mmol}, 70 \%)$. Analytical data: ${ }^{31}{ }^{1} \mathbf{H}$ NMR (400 MHz, $\left.\mathrm{CDCl}_{3}, 298 \mathrm{~K}\right): \delta_{\mathrm{H}} 8.17-8.13(\mathrm{~m}, 1 \mathrm{H}), 8.00-7.94(\mathrm{~m}, 1 \mathrm{H}), 7.63-7.59(\mathrm{~m}, 1 \mathrm{H})$, 7.58-7.53 (m, 1H), 7.43-7.38 (m, 1H) ppm; ${ }^{13} \mathbf{C}$ NMR (100 MHz, $\left.\mathrm{CDCl}_{3}, 298 \mathrm{~K}\right): \delta_{\mathrm{C}} 144.0$, $139.5,129.2,128.3,126.3,123.9,123.6,119.5,108.1 \mathrm{ppm} ; \mathbf{R}_{\mathbf{f}}(9: 1$ hexanes/EtOAc; $\left.\mathrm{UV} / \mathrm{KMnO}_{4}\right): 0.50$.<smiles>Nc1csc2ccccc12</smiles>

Benzo[b] thiophene-3-carbonitrile (2z) (CAS: 24434-84-2): According to General Procedure A, the product was prepared using the following amounts of reagent: $\mathrm{NiBr}_{2}(\mathrm{bpy}) \cdot \mathrm{xH}_{2} \mathrm{O}(15 \mathrm{mg}$, $0.040 \mathrm{mmol}, 10 \mathrm{~mol} \%$ ), $\mathrm{Zn}(0)$ dust ( $52 \mathrm{mg}, 0.80 \mathrm{mmol}, 2.0$ equiv), 3-bromobenzothiophene (52 $\mu \mathrm{L}, 0.40 \mathrm{mmol}, 1.0$ equiv), and MPMN (2.0 $\mathrm{mL}$ of a $0.24 \mathrm{M}$ stock solution in DMA, $0.48 \mathrm{mmol}$, 1.2 equiv). The crude residue was purified by flash column chromatography (gradient of $0-15 \%$ acetone/hexanes) to yield $\mathbf{2 z}$ as a white solid (48 $\mathrm{mg}, 0.300 \mathrm{mmol}, 75 \%$ ). Analytical data: ${ }^{31}{ }^{1} \mathbf{H}$ NMR (500 MHz, $\left.\mathrm{CDCl}_{3}, 298 \mathrm{~K}\right): \delta_{\mathrm{H}} 8.13(\mathrm{~s}, 1 \mathrm{H}), 8.03-8.98(\mathrm{~m}, 1 \mathrm{H}), 7.94-7.89(\mathrm{~m}, 1 \mathrm{H}), 7.58-$ 7.46 (m, 2H) ppm; ${ }^{13} \mathbf{C}$ NMR $\left(125 \mathrm{MHz}, \mathrm{CDCl}_{3}, 298 \mathrm{~K}\right): \delta_{\mathrm{C}} 138.6,137.7,137.4,126.3,126.1$, 123.0, 122.7, 114.5, 107.3 ppm; $\mathbf{R}_{\mathbf{f}}$ (9:1 hexanes/EtOAc; UV): 0.40. 


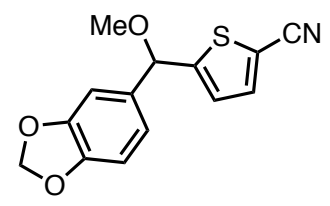

5-(Benzo[1,3]dioxol-5-yl(methoxy)methyl)thiophene-2-carbonitrile (2an): According to General Procedure A, the product was prepared using the following amounts of reagent: $\mathrm{NiBr}_{2}$ (bpy) $\mathrm{xH}_{2} \mathrm{O}$ (15 mg, $0.040 \mathrm{mmol}, 10 \mathrm{~mol} \%$ ), $\mathrm{Zn}(0)$ dust (52 mg, $0.80 \mathrm{mmol}, 2.0$ equiv), $\mathrm{S} 18$ (0.13 g, $0.40 \mathrm{mmol}, 1.0$ equiv), and MPMN (2.0 $\mathrm{mL}$ of a $0.24 \mathrm{M}$ stock solution in DMA, $0.48 \mathrm{mmol}, 1.2$ equiv). The crude residue was purified by flash column chromatography (gradient of 5-30\% EtOAc/hexanes) to yield 2an as a yellow solid (trial 1: $49 \mathrm{mg}, 0.179 \mathrm{mmol}$, 45\%; trial 2: $20 \mathrm{mg}, 0.072 \mathrm{mmol}, 18 \%)$. Analytical data: ${ }^{1} \mathbf{H} \mathbf{N M R}\left(400 \mathrm{MHz}, \mathrm{CDCl}_{3}, 298 \mathrm{~K}\right): \delta_{\mathrm{H}}$ 7.47-7.40 (m, 1H), 6.87-6.77 (m, 3H), 6.77-6.71 (m, 1H), 6.02-5.92 (m, 2H), $5.32(\mathrm{~s}, 1 \mathrm{H}), 3.37$ $(\mathrm{s}, 3 \mathrm{H}) \mathrm{ppm} ;{ }^{13} \mathbf{C}$ NMR $\left(100 \mathrm{MHz}, \mathrm{CDCl}_{3}, 298 \mathrm{~K}\right): \delta_{\mathrm{C}} 155.3,148.4,148.2,137.4,133.7,124.8$, 121.1, 114.5, 109.2, 108.4, 107.2, 101.5, 81.2, 57.1 ppm; IR (neat): 2990, 2931, 2897, 2824, 2778, 2218, 1502, 1485, 1439, 1239, 1188, 1095, 1079, 1035, 965, 927, 867, 809, 784, $687 \mathrm{~cm}^{-1}$; $\mathbf{R}_{\mathbf{f}}(3 \%$ EtOAc/hexanes; UV/p-anisaldehyde): 0.23 .<smiles>N#Cc1cnn(Cc2ccccc2)c1</smiles>

1-Benzyl-1H-pyrazole-4-carbonitrile (2ao) (CAS: 121358-86-9): According to General Procedure $\mathrm{A}$, the product was prepared using the following amounts of reagent:

$\mathrm{NiBr}_{2}$ (bpy) $\mathrm{xH}_{2} \mathrm{O}$ (15 mg, $0.040 \mathrm{mmol}, 10 \mathrm{~mol} \%$ ), Zn(0) dust (52 mg, $0.80 \mathrm{mmol}, 2.0$ equiv), S19 (66 $\mu \mathrm{L}, 0.40 \mathrm{mmol}, 1.0$ equiv), and MPMN (2.0 mL of a $0.24 \mathrm{M}$ stock solution in DMA, $0.48 \mathrm{mmol}, 1.2$ equiv). The crude residue was purified by flash column chromatography (gradient of 0-20\% EtOAc/hexanes) to yield $2 \mathbf{2 a o}$ as a white solid (16 mg, $0.087 \mathrm{mmol}, 22 \%$ ). Analytical data: ${ }^{32}{ }^{1} \mathbf{H}$ NMR $\left(500 \mathrm{MHz}, \mathrm{CDCl}_{3}, 298 \mathrm{~K}\right): \delta_{\mathrm{H}} 7.84-7.80(\mathrm{~m}, 1 \mathrm{H}), 7.76-7.74(\mathrm{~m}$, 1H), 7.42-7.34 (m, 3H), 7.27-7.22 (m, 2H), 5.32 (s, 2H) ppm; ${ }^{13} \mathbf{C}$ NMR (125 MHz, CDCl, 298 $\mathrm{K}): \delta_{\mathrm{C}} 142.6,134.5,134.3,129.3,129.0,128.2,113.4,92.8,56.9$ ppm; $\mathbf{R}_{\mathbf{f}}(9: 1$ hexanes/EtOAc; $\left.\mathrm{UV} / \mathrm{KMnO}_{4}\right): 0.33$.<smiles>CN1C(=O)C(=O)c2cc(C#N)ccc21</smiles>

1-Methyl-2,3-dioxoindoline-5-carbonitrile (2ap) (CAS: 184904-71-0): According to General Procedure A, the product was prepared using the following amounts of reagent:

$\mathrm{NiBr}_{2}$ (bpy) $\bullet \mathrm{xH}_{2} \mathrm{O}$ (15 mg, $0.040 \mathrm{mmol}, 10 \mathrm{~mol} \%$ ), Zn(0) dust (52 mg, $0.80 \mathrm{mmol}, 2.0$ equiv), S20 (96 mg, $0.40 \mathrm{mmol}, 1.0$ equiv), and MPMN (2.0 $\mathrm{mL}$ of a $0.24 \mathrm{M}$ stock solution in DMA, $0.48 \mathrm{mmol}, 1.2$ equiv). The crude residue was purified by flash column chromatography (gradient of 40-100\% EtOAc/hexanes) to yield 2ap as an orange-red solid (trial 1: $16 \mathrm{mg}, 0.086$ mmol, 22\%; trial 2: $11 \mathrm{mg}, 0.059 \mathrm{mmol}, 15 \%)$. Analytical data: ${ }^{33}{ }^{1} \mathbf{H} \mathbf{N M R}\left(400 \mathrm{MHz}, \mathrm{CDCl}_{3}\right.$, $298 \mathrm{~K}): \delta_{\mathrm{H}} 7.94-7.84(\mathrm{~m}, 2 \mathrm{H}), 7.03(\mathrm{~d}, J=8.2 \mathrm{~Hz}, 1 \mathrm{H}), 3.32(\mathrm{~s}, 3 \mathrm{H}) \mathrm{ppm} ;{ }^{13} \mathbf{C}$ NMR $(100 \mathrm{MHz}$, $\left.\mathrm{CDCl}_{3}, 298 \mathrm{~K}\right): \delta_{\mathrm{C}} 181.2,157.6,154.1,142.1,128.8,117.7,117.5,110.9,107.9,26.8$ ppm; $\mathbf{R}_{\mathbf{f}}$ (1:1 EtOAc/hexanes; UV): 0.16. 
<smiles>N#Cc1cnc2ccccc2c1</smiles>

Quinoline-3-carbonitrile (2aq) (CAS: 34846-64-5): According to General Procedure A, the product was prepared using the following amounts of reagent: $\mathrm{NiBr}_{2}(\mathrm{bpy}) \cdot \mathrm{xH}_{2} \mathrm{O}(15 \mathrm{mg}, 0.040$ $\mathrm{mmol}, 10 \mathrm{~mol} \%), \mathrm{Zn}(0)$ dust (52 $\mathrm{mg}, 0.80 \mathrm{mmol}, 2.0$ equiv), 3-bromoquinoline ( $54 \mu \mathrm{L}, 0.40$ mmol, 1.0 equiv), and MPMN (2.0 mL of a $0.24 \mathrm{M}$ stock solution in DMA, $0.48 \mathrm{mmol}, 1.2$ equiv). The crude residue was purified by flash column chromatography (gradient of 20-50\% EtOAc/hexanes) to yield 2aq as a white solid (trial 1: $17 \mathrm{mg}, 0.110 \mathrm{mmol}, 28 \%$; trial 2: $23 \mathrm{mg}$, $0.148 \mathrm{mmol}, 37 \%)$. Analytical data: ${ }^{34}{ }^{1} \mathrm{H}$ NMR $\left(500 \mathrm{MHz}, \mathrm{CDCl}_{3}, 298 \mathrm{~K}\right): \delta_{\mathrm{H}} 9.07-9.04(\mathrm{~m}$, 1H), 8.58-8.53 (m, 1H), 8.22-8.15 (m, 1H), 7.94-7.86 (m, 2H), 7.74-7.67 (m, 1H) ppm; ${ }^{13} \mathbf{C}$ NMR (125 MHz, $\left.\mathrm{CDCl}_{3}, 298 \mathrm{~K}\right): \delta_{\mathrm{C}} 149.7,148.8,141.5,132.8,129.8,128.5,128.3,126.2$, 117.1, 106.6 ppm; $\mathbf{R}_{\mathbf{f}}(9: 1$ hexanes/EtOAc; UV): 0.12.<smiles>N#Cc1cccc2ncccc12</smiles>

Quinoline-5-carbonitrile (2ae) (CAS: 59551-02-9): According to General Procedure A, the product was prepared using the following amounts of reagent: $\mathrm{NiBr}_{2}(\mathrm{bpy}) \bullet \mathrm{xH}_{2} \mathrm{O}(15 \mathrm{mg}, 0.040$ mmol, $10 \mathrm{~mol} \%$ ), $\mathrm{Zn}(0)$ dust (52 $\mathrm{mg}, 0.80 \mathrm{mmol}, 2.0$ equiv), 5-bromoquinoline ( $83 \mathrm{mg}, 0.40$ mmol, 1.0 equiv), and MPMN (2.0 mL of a $0.24 \mathrm{M}$ stock solution in DMA, $0.48 \mathrm{mmol}, 1.2$ equiv). The crude residue was purified by flash column chromatography (gradient of 0-60\% EtOAc/hexanes) to yield 2ae as a white solid (trial 1: $34 \mathrm{mg}, 0.221 \mathrm{mmol}, 55 \%$; trial 2: $32 \mathrm{mg}$, $0.208 \mathrm{mmol}, 52 \%)$. Analytical data: ${ }^{10}{ }^{1} \mathrm{H}$ NMR (400 MHz, $\left.\mathrm{CDCl}_{3}, 298 \mathrm{~K}\right): \delta_{\mathrm{H}} 9.11-9.01(\mathrm{~m}$, $1 \mathrm{H}), 8.61-8.51(\mathrm{~m}, 1 \mathrm{H}), 8.43-8.33(\mathrm{~m}, 1 \mathrm{H}), 8.05-7.95(\mathrm{~m}, 1 \mathrm{H}), 7.84-7.74(\mathrm{~m}, 1 \mathrm{H}), 7.67-7.58$ $(\mathrm{m}, 1 \mathrm{H}) \mathrm{ppm} ;{ }^{13} \mathbf{C}$ NMR $\left(100 \mathrm{MHz}, \mathrm{CDCl}_{3}, 298 \mathrm{~K}\right): \delta_{\mathrm{C}} 152.1,147.6,135.2,133.6,133.2,128.9$, 128.2, 123.4, 116.8, 110.6 ppm; $\mathbf{R}_{\mathbf{f}}(9: 1$ hexanes/EtOAc; UV): 0.07.<smiles>COc1ccc(C#N)cn1</smiles>

6-Methoxynicotinonitrile (2ar) (CAS: 15871-85-9): According to General Procedure A, the product was prepared using the following amounts of reagent: $\mathrm{NiBr}_{2}(\mathrm{bpy}) \bullet \mathrm{xH}_{2} \mathrm{O}(15 \mathrm{mg}, 0.040$ mmol, $10 \mathrm{~mol} \%$ ), $\mathrm{Zn}(0)$ dust (52 mg, $0.80 \mathrm{mmol}, 2.0$ equiv), 5-bromo-2-methoxypyridine (52 $\mu \mathrm{L}, 0.40 \mathrm{mmol}, 1.0$ equiv), and MPMN (2.0 mL of a $0.24 \mathrm{M}$ stock solution in DMA, $0.48 \mathrm{mmol}$, 1.2 equiv). The crude residue was purified by flash column chromatography (gradient of 5-30\% EtOAc/hexanes) to yield $2 \mathrm{ar}$ as an off-white solid (19 mg, $0.144 \mathrm{mmol}, 36 \%)$. Analytical data: ${ }^{26}$ ${ }^{1} \mathbf{H}$ NMR $\left(400 \mathrm{MHz}, \mathrm{CDCl}_{3}, 298 \mathrm{~K}\right): \delta_{\mathrm{H}} 8.49(\mathrm{dd}, J=2.4,0.8 \mathrm{~Hz}, 1 \mathrm{H}), 7.77(\mathrm{dd}, J=8.7,2.3 \mathrm{~Hz}$, $1 \mathrm{H}), 6.82(\mathrm{dd}, J=8.7,0.8 \mathrm{~Hz}, 1 \mathrm{H}), 4.00(\mathrm{~s}, 3 \mathrm{H}) \mathrm{ppm} ;{ }^{13} \mathbf{C ~ N M R}\left(100 \mathrm{MHz}, \mathrm{CDCl}_{3}, 298 \mathrm{~K}\right): \delta_{\mathrm{C}}$ 166.2, 152.1, 141.0, 117.4, 112.0, 102.6, 54.4 ppm; $\mathbf{R}_{\mathbf{f}}$ (3\% EtOAc/hexanes; UV): 0.23.<smiles>N#Cc1ccc(N2CCCCC2)nc1</smiles>

6-(Piperidin-1-yl)nicotinonitrile (2ab) (CAS: 501378-38-7): According to General Procedure A, the product was prepared using the following amounts of reagent: $\mathrm{NiBr}_{2}(\mathrm{bpy}) \bullet \times \mathrm{H}_{2} \mathrm{O}(15 \mathrm{mg}$, $0.040 \mathrm{mmol}, 10 \mathrm{~mol} \%), \mathrm{Zn}(0)$ dust (52 mg, $0.80 \mathrm{mmol}, 2.0$ equiv), S21 (68 $\mu \mathrm{L}, 0.40 \mathrm{mmol}, 1.0$ 
equiv), and MPMN (2.0 mL of a $0.24 \mathrm{M}$ stock solution in DMA, $0.48 \mathrm{mmol}, 1.2$ equiv). The crude residue was purified by flash column chromatography (gradient of 5-30\% EtOAc/hexanes) to yield $\mathbf{2 a b}$ as a colourless oil (58 $\mathrm{mg}, 0.310 \mathrm{mmol}, 78 \%)$. Analytical data: ${ }^{35}{ }^{1} \mathbf{H}$ NMR (400 $\left.\mathrm{MHz}, \mathrm{CDCl}_{3}, 298 \mathrm{~K}\right):$ 8.39-8.34 (m, 1H), 7.57-7.52 (m, 1H), 6.59-6.54 (m, 1H), 3.69-3.59 (m, 4H), 1.74-1.57 (m, 6H) ppm; ${ }^{13} \mathbf{C}$ NMR (100 MHz, $\left.\mathrm{CDCl}_{3}, 298 \mathrm{~K}\right): \delta_{\mathrm{C}} 159.4,152.9,139.7$, 119.1, 105.7, 95.3, 45.8, 25.6, 24.7 ppm; $\mathbf{R}_{\mathbf{f}}(9: 1$ hexanes/EtOAc; UV): 0.23.<smiles>N#Cc1cnc(N2CCCCCC2)nc1</smiles>

2-(Azepan-1-yl)pyrimidine-5-carbonitrile (2ac) (CAS: 1774902-18-9): According to General Procedure $\mathrm{A}$, the product was prepared using the following amounts of reagent: $\mathrm{NiBr}_{2}$ (bpy) $x_{2} \mathrm{O}$ (15 mg, $0.040 \mathrm{mmol}, 10 \mathrm{~mol} \%$ ), $\mathrm{Zn}(0)$ dust (52 mg, $0.80 \mathrm{mmol}, 2.0$ equiv), S22 (0.11 g, $0.40 \mathrm{mmol}, 1.0$ equiv), and MPMN (2.0 $\mathrm{mL}$ of a $0.24 \mathrm{M}$ stock solution in DMA, $0.48 \mathrm{mmol}, 1.2$ equiv). The crude residue was purified by flash column chromatography (gradient of 0-30\% EtOAc/hexanes) to yield 2ac as a white solid (52 $\mathrm{mg}, 0.257 \mathrm{mmol}, 64 \%$ ). Analytical data: ${ }^{1} \mathbf{H}$ NMR $\left(400 \mathrm{MHz}, \mathrm{CDCl}_{3}, 298 \mathrm{~K}\right): \delta_{\mathrm{H}} 8.45(\mathrm{~s}, 2 \mathrm{H}), 3.82-3.72(\mathrm{~m}, 4 \mathrm{H}), 1.83-$ 1.70 (m, 4H), 1.60-1.49 (m, 4H) ppm; ${ }^{13} \mathbf{C}$ NMR (100 MHz, $\left.\mathrm{CDCl}_{3}, 298 \mathrm{~K}\right): \delta_{\mathrm{C}} 160.7,160.7$, 117.4, 95.0, 48.0, 27.5, $27.1 \mathrm{ppm}$; HRMS $m / z$ (DART): calcd for $\mathrm{C}_{11} \mathrm{H}_{15} \mathrm{~N}_{4}$ : 203.1297; found 203.1293; IR (neat): 2934, 2854, 2218, 1602, 1510, 1439, 1206, 963, $792 \mathrm{~cm}^{-1}$; m.p.: 43-51 ${ }^{\circ} \mathrm{C}$; $\mathbf{R}_{\mathbf{f}}(9: 1$ hexanes/EtOAc; UV): 0.29.<smiles>N#Cc1cnc(N2CC[Nb](=O)C2)nc1</smiles>

2-(4-Benzylpiperazin-1-yl)pyrimidine-5-carbonitrile (2ad): According to General Procedure A, the product was prepared using the following amounts of reagent: $\mathrm{NiBr}_{2}(\mathrm{bpy}) \bullet \times \mathrm{H}_{2} \mathrm{O}(15 \mathrm{mg}$, $0.040 \mathrm{mmol}, 10 \mathrm{~mol} \%$ ), $\mathrm{Zn}(0)$ dust (52 mg, $0.80 \mathrm{mmol}, 2.0$ equiv), $\mathbf{S 2 3}$ ( $0.13 \mathrm{~g}, 0.40 \mathrm{mmol}, 1.0$ equiv), and MPMN (2.0 mL of a $0.24 \mathrm{M}$ stock solution in DMA, $0.48 \mathrm{mmol}, 1.2$ equiv). The crude residue was purified by flash column chromatography (gradient of $20-50 \%$ EtOAc/hexanes) to yield 2ad as a colourless oil (trial 1: $39 \mathrm{mg}, 0.223 \mathrm{mmol}, 56 \%$; trial 2: $66 \mathrm{mg}$, $0.236 \mathrm{mmol}, 59 \%)$. Analytical data: ${ }^{1} \mathbf{H}$ NMR (400 MHz, $\left.\mathrm{CDCl}_{3}, 298 \mathrm{~K}\right): \delta_{\mathrm{H}} 8.49-8.43(\mathrm{~m}, 2 \mathrm{H})$, 7.38-7.23 (m, 5H), 3.95-3.88 (m, 4H), $3.54(\mathrm{~s}, 2 \mathrm{H}), 2.55-2.46(\mathrm{~m}, 4 \mathrm{H}) \mathrm{ppm} ;{ }^{13} \mathbf{C}$ NMR (100 $\left.\mathrm{MHz}, \mathrm{CDCl}_{3}, 298 \mathrm{~K}\right): \delta_{\mathrm{C}} 160.8,160.5,137.7,129.3,128.5,127.4,117.0,95.5,63.1,52.8,44.1$ ppm; HRMS $m / z$ (DART): calcd for $\mathrm{C}_{16} \mathrm{H}_{18} \mathrm{~N}_{5}(\mathrm{M}+\mathrm{H}): 280.1562$; found 280.1556; IR (neat): 3439, 2923, 2814, 2767, 2219, 1600, 1525, 1509, 1435, 1411, 1402, 1360, 1343, 1314, 1262, 1226, 1190, 1134, 1109, 1077, 1040, 1017, 984, 951, 914, 797, 786, 743, 699, $659 \mathrm{~cm}^{-1} ; \mathbf{R}_{\mathbf{f}}(9: 1$ hexanes/EtOAc; UV): 0.09 . 


\section{E.2. Synthesis of biologically relevant benzonitriles}<smiles>CNCCCN1c2ccccc2Sc2ccc(C#N)cc21</smiles>

10-(3-(Dimethylamino)propyl)-10H-phenothiazine-2-carbonitrile (cyanopromazine) (2af) (CAS: 7678-64-0): According to General Procedure A, the product was prepared using the following amounts of reagent: $\mathrm{NiBr}_{2}(\mathrm{bpy}) \cdot \mathrm{xH}_{2} \mathrm{O}(7.5 \mathrm{mg}, 0.020 \mathrm{mmol}, 10 \mathrm{~mol} \%), \mathrm{Zn}(0)$ dust (26 mg, $0.40 \mathrm{mmol}, 2.0$ equiv), $\mathbf{S 2 7}$ (73 mg, $0.20 \mathrm{mmol}, 1.0$ equiv), and MPMN (1.0 mL of a $0.24 \mathrm{M}$ stock solution in DMA, $0.48 \mathrm{mmol}, 1.2$ equiv). The crude residue was purified by flash column chromatography (gradient of $0 / 0 / 100$ to $9 / 1 / 90 \mathrm{MeOH} / \mathrm{NEt}_{3} / \mathrm{DCM}$ ) to yield $\mathbf{2 a f}$ as a yellow solid, which sample contained a small $(<5 \%)$ amount of promazine $(30 \mathrm{mg}, 0.097 \mathrm{mmol}$, 49\%). Analytical data: ${ }^{361} \mathbf{H}$ NMR $\left(400 \mathrm{MHz}, \mathrm{CDCl}_{3}, 298 \mathrm{~K}\right): \delta_{\mathrm{H}} 7.21-7.12(\mathrm{~m}, 3 \mathrm{H}), 7.13-7.05$ (m, 2H), 7.00-7.91 (m, 2H), $3.89(\mathrm{dd}, J=8.0,6.7 \mathrm{~Hz}, 2 \mathrm{H}), 2.39$ (t, $J=6.8 \mathrm{~Hz}, 2 \mathrm{H}), 2.21(\mathrm{~s}, 6 \mathrm{H})$, $(\mathrm{t}, J=7.0 \mathrm{~Hz}, 2 \mathrm{H}) \mathrm{ppm} ;{ }^{13} \mathbf{C}$ NMR $\left(100 \mathrm{MHz}, \mathrm{CDCl}_{3}, 298 \mathrm{~K}\right): 145.9,144.0,132.3,128.0,127.8$, $127.7,126.0,123.6,123.5,119.2,118.1,116.1,110.9,57.0,45.7,45.7,25.1 \mathrm{ppm} ; \mathbf{R}_{\mathbf{f}}(10 \%$ $\left.\mathrm{MeOH} / \mathrm{DCM} ; \mathrm{UV} / \mathrm{KMnO}_{4}\right): 0.22$.<smiles>CC(C)(C)OCC(=O)N1CCN(c2ccc(C#N)cc2)CC1</smiles>

4-(4-(2-((tert-Butyldimethylsilyl)oxy)acetyl)piperazin-1-yl)benzonitrile (2ag): According to General Procedure A, 2ag was prepared using the following amounts of reagent: $\mathrm{NiBr}_{2}$ (bpy)•xH $\mathrm{H}_{2} \mathrm{O}$ (7.5 mg, $0.020 \mathrm{mmol}, 10 \mathrm{~mol} \%$ ), $\mathrm{Zn}(0)$ dust (26 mg, $0.40 \mathrm{mmol}, 2.0$ equiv), S31 ( $83 \mathrm{mg}, 0.20 \mathrm{mmol}, 1.0$ equiv), and MPMN (1.0 mL of a $0.24 \mathrm{M}$ stock solution in DMA, $0.48 \mathrm{mmol}, 1.2$ equiv). The crude residue was purified by flash column chromatography (gradient of 30-80\% EtOAc/hexanes) to yield 2ag as a white solid, which was a mixture of conformational isomers (49 mg, $0.136 \mathrm{mmol}, 68 \%$ ). Analytical data: ${ }^{1} \mathbf{H}$ NMR $(400 \mathrm{MHz}$, $\left.\mathrm{CDCl}_{3}, 298 \mathrm{~K}\right): 7.55-7.48(\mathrm{~m}, 1.5 \mathrm{H}$, major), $7.32-7.25(\mathrm{~m}, 0.5 \mathrm{H}$, minor), $6.96-6.89(\mathrm{~m}, 0.5 \mathrm{H}$, minor), 6.90-6.83 (m, 1.5H, major), 4.33 (s, 2H), 3.82-3.68 (m, 4H), 3.40-3.28 (m, 3H, major), 3.22-3.13 (m, 1H, minor), 0.91 (s, 9H), 0.12 (s, 6H) ppm; $\left.{ }^{13} \mathbf{C ~ N M R ~ ( 1 0 0 ~ M H z , ~ C D C l ~} 3,298 \mathrm{~K}\right)$ : $169.5,153.2,133.8,129.4,116.8,114.8,64.4,55.8,47.2,25.9,18.4,-5.3 \mathrm{ppm} ; \mathbf{H R M S ~} \mathrm{m} / \mathrm{z}$ (DART): calcd for $\mathrm{C}_{19} \mathrm{H}_{30} \mathrm{~N}_{3} \mathrm{O}_{2} \mathrm{Si}(\mathrm{M}+\mathrm{H})$ : 360.2089; found: 360.2098; IR (neat): 2955, 2928, 2883, 2856, 2219, 1652, 1605, 1520, 1395, 1244, 1183, 1149, 1028, 831, 810, $776 \mathrm{~cm}^{-1}$; m.p.: $69-71{ }^{\circ} \mathrm{C}$. 


\section{E.3. Synthesis of Synthesis of benzonitriles from alternate electrophiles using $\mathrm{NaBr}$}

General Procedure B: Synthesis of benzonitriles from alternate electrophiles using $\mathrm{NaBr}$

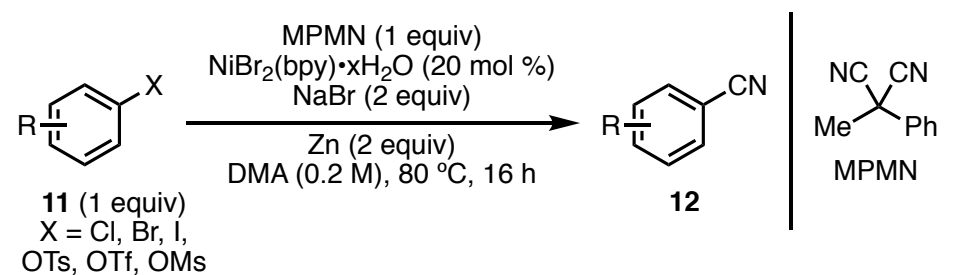

Caution: Depending on the reductant, ligand, and nickel catalyst used, decyanation of excess MPMN was sometimes observed, implying formation of cyanide salts in situ. As a precaution, all reactions were handled under basic conditions and disposed of appropriately.

To a flame-dried 8-mL culture tube with a stir bar was added zinc( 0$)$ dust $(52 \mathrm{mg}, 0.80 \mathrm{mmol}$, 2.0 equiv), aryl halide (if solid; $0.40 \mathrm{mmol}, 1.0$ equiv), $\mathrm{NiBr}_{2}(\mathrm{bpy}) \cdot \mathrm{xH}_{2} \mathrm{O}$ (30 mg, $0.080 \mathrm{mmol}$, $20 \mathrm{~mol} \%$ ), and sodium bromide ( $82 \mathrm{mg}, 0.80 \mathrm{mmol}, 2.0$ equiv). The tube was sealed with a size19 septum and electrical tape and was evacuated and backfilled with $\mathrm{N}_{2}(\times 3)$. DMA $(1.0 \mathrm{~mL})$ was added. If the aryl halide was an oil, aryl halide was then added $(0.40 \mathrm{mmol}, 1.0$ equiv). The reaction was stirred at r.t. for $2 \mathrm{~min}$. Then, 2-methyl-2-phenylmalononitrile $(1.0 \mathrm{~mL}$ of a $0.40 \mathrm{M}$ stock solution in DMA, $0.40 \mathrm{mmol}, 1.0$ equiv; total reaction volume $=2.0 \mathrm{~mL}, 0.20 \mathrm{M}$ ) was added and the reaction was immediately put into an oil bath at $80^{\circ} \mathrm{C}$ and stirred for $16 \mathrm{~h}$. The reaction was cooled to r.t., quenched with sat. aq. $\mathrm{NaHCO}_{3}$, and $n$-dodecane $(91 \mu \mathrm{L}, 0.40 \mathrm{mmol}$, 1.0 equiv) was added as an internal standard for GC-MS analysis. The solution was extracted with EtOAc ( $\times 3)$ and the organic fractions were combined, filtered over a plug of $\mathrm{MgSO}_{4}$ and Celite, and concentrated. The crude residue was purified by flash column chromatography to yield the desired benzonitrile.<smiles>N#Cc1ccc2c(c1)CCCC2</smiles>

5,6,7,8-Tetrahydronaphthalene-2-carbonitrile (from ArOTs) (12a) (CAS: 17104-67-5): According to General Procedure B, 12a was prepared using the following amounts of reagent: $\mathrm{NiBr}_{2}$ (bpy) $\mathrm{xH}_{2} \mathrm{O}(30 \mathrm{mg}, 0.080 \mathrm{mmol}, 20 \mathrm{~mol} \%$ ), Zn(0) dust (52 mg, $0.80 \mathrm{mmol}, 2.0$ equiv), S33 ( $0.12 \mathrm{~g}, 0.40 \mathrm{mmol}, 1.0$ equiv), sodium bromide ( $82 \mathrm{mg}, 0.80 \mathrm{mmol}, 2.0$ equiv), DMA (1.0 $\mathrm{mL}$ ), and MPMN (1.0 mL of a $0.40 \mathrm{M}$ stock solution in DMA, $0.40 \mathrm{mmol}, 1.0$ equiv). The crude residue was purified by flash column chromatography (gradient of 0-10\% EtOAc/hexanes) to yield 12a as a colourless oil (trial 1: $36 \mathrm{mg}, 0.228 \mathrm{mmol}, 57 \%$; trial 2: $31 \mathrm{mg}, 0.200 \mathrm{mmol}, 50 \%$ ). Exposing this substrate to identical conditions in the absence of sodium bromide gave no yield of 12a, as determined by GC-MS. Analytical data: ${ }^{10} \mathbf{H} \mathbf{N M R}\left(500 \mathrm{MHz}, \mathrm{CDCl}_{3}, 298 \mathrm{~K}\right): \delta_{\mathrm{H}} 7.36-$ $7.30(\mathrm{~m}, 2 \mathrm{H}), 7.15-7.10(\mathrm{~m}, 1 \mathrm{H}), 2.86-2.72(\mathrm{~m}, 4 \mathrm{H}), 1.86-1.76(\mathrm{~m}, 4 \mathrm{H}) \mathrm{ppm} ;{ }^{13} \mathbf{C}$ NMR $(125$ $\left.\mathrm{MHz}, \mathrm{CDCl}_{3}, 298 \mathrm{~K}\right): \delta_{\mathrm{C}} 143.0,138.4,132.8,129.8,128.8,119.4,109.1,29.6,29.0,22.6,22.5$ ppm; $\mathbf{R}_{\mathbf{f}}(9: 1$ hexanes/EtOAc; UV): 0.58.<smiles>N#Cc1ccc(-c2ccccc2)cc1</smiles> 
[1,1'-Biphenyl]-4-carbonitrile (from ArOMs) (12b) (CAS: 2920-38-9): According to General Procedure $\mathrm{B}, \mathbf{1 2 b}$ was prepared using the following amounts of reagent: $\mathrm{NiBr}_{2}(\mathrm{bpy}) \cdot \mathrm{xH}_{2} \mathrm{O}(30$ $\mathrm{mg}, 0.080 \mathrm{mmol}, 20 \mathrm{~mol} \%), \mathrm{Zn}(0)$ dust (52 mg, $0.80 \mathrm{mmol}, 2.0$ equiv), S34 (99 mg, $0.40 \mathrm{mmol}$, 1.0 equiv), sodium bromide ( $82 \mathrm{mg}, 0.80 \mathrm{mmol}, 2.0$ equiv), DMA (1.0 mL), and MPMN (1.0 mL of a $0.40 \mathrm{M}$ stock solution in DMA, $0.40 \mathrm{mmol}, 1.0$ equiv). The crude residue was purified by flash column chromatography (gradient of 0-20\% EtOAc/hexanes) to yield 12b as a white solid (36 mg, $0.201 \mathrm{mmol}, 50 \%$ ). Exposing this substrate to identical conditions in the absence of sodium bromide gave no yield of $\mathbf{1 2 b}$, as determined by GC-MS. Analytical data: ${ }^{10} \mathbf{1} \mathbf{H}$ NMR $\left(500 \mathrm{MHz}, \mathrm{CDCl}_{3}, 298 \mathrm{~K}\right): \delta_{\mathrm{H}} 7.76-7.71(\mathrm{~m}, 2 \mathrm{H}), 7.71-7.67(\mathrm{~m}, 2 \mathrm{H}), 7.62-7.57(\mathrm{~m}, 2 \mathrm{H}), 7.52-$ $7.46(\mathrm{~m}, 2 \mathrm{H}), 7.45-7.40(\mathrm{~m}, 1 \mathrm{H}),{ }^{13} \mathbf{C} \mathbf{N M R}\left(125 \mathrm{MHz}, \mathrm{CDCl}_{3}, 298 \mathrm{~K}\right): \delta_{\mathrm{C}} 145.8,139.3,132.7$, 129.3, 128.8, 127.9, 127.4, 119.1, 111.1 ppm; $\mathbf{R}_{\mathbf{f}}(9: 1$ hexanes/EtOAc; UV): 0.53.<smiles>CC1(C)COC(c2ccc(C#N)cc2)OC1</smiles>

4-(5,5-Dimethyl-1,3-dioxan-2-yl)benzonitrile (from ArCl) (12c) (CAS: 106456-92-2): According to General Procedure B, 12c was prepared using the following amounts of reagent: $\mathrm{NiBr}_{2}$ (bpy) $)_{2} \cdot 2 \mathrm{H}_{2} \mathrm{O}$ (45 mg, $0.080 \mathrm{mmol}, 20 \mathrm{~mol} \%$ ), Zn(0) dust (52 mg, $0.80 \mathrm{mmol}, 2.0$ equiv), S35 (91 mg, $0.40 \mathrm{mmol}, 1.0$ equiv), sodium bromide ( $82 \mathrm{mg}, 0.80 \mathrm{mmol}, 2.0$ equiv), DMA (1.0 mL), and MPMN (1.0 mL of a $0.40 \mathrm{M}$ stock solution in DMA, $0.40 \mathrm{mmol}, 1.0$ equiv). The crude residue was purified by flash column chromatography (gradient of 0-20\% EtOAc/hexanes) to yield $12 \mathrm{c}$ as a white solid (51 $\mathrm{mg}, 0.235 \mathrm{mmol}, 59 \%)$. Exposing this substrate to identical conditions in the absence of sodium bromide gave no yield of 12c, as determined by GC-MS. Analytical data: ${ }^{37}{ }^{1} \mathbf{H}$ NMR $\left(400 \mathrm{MHz}, \mathrm{CDCl}_{3}, 298 \mathrm{~K}\right): \delta_{\mathrm{H}} 7.72-7.52(\mathrm{~m}, 4 \mathrm{H}), 5.42(\mathrm{~s}, 1 \mathrm{H})$, 3.84-3.72 (m, 2H), 3.72-3.59 (m, 2H), 1.27 (s, 3H), 0.81 (s, 3H) ppm; ${ }^{13} \mathbf{C}$ NMR (100 MHz, $\left.\mathrm{CDCl}_{3}, 298 \mathrm{~K}\right): \delta_{\mathrm{C}} 143.2,132.2,127.0,118.7,112.6,100.3,77.7,30.3,23.0,21.8$ ppm; $\mathbf{R}_{\mathbf{f}}(8: 2$ hexanes/EtOAc; $\left.\mathrm{UV} / \mathrm{KMnO}_{4}\right): 0.56$.<smiles>COc1cccc(C#N)c1</smiles>

3-Methoxybenzonitrile (from ArOTf) (2a) (CAS: 1527-89-5): According to General Procedure B, 2a was prepared using the following amounts of reagent: $\mathrm{NiBr}_{2}$ (bpy) $\bullet \mathrm{xH}_{2} \mathrm{O}(30 \mathrm{mg}$, $0.080 \mathrm{mmol}, 20 \mathrm{~mol} \%), \mathrm{Zn}(0)$ dust (52 mg, $0.80 \mathrm{mmol}, 2.0$ equiv), S32 ( $0.10 \mathrm{~g}, 0.40 \mathrm{mmol}$, 1.0 equiv), sodium bromide ( $82 \mathrm{mg}, 0.80 \mathrm{mmol}, 2.0$ equiv), DMA (1.0 mL), and MPMN (1.0 mL of a $0.40 \mathrm{M}$ stock solution in DMA, $0.40 \mathrm{mmol}, 1.0$ equiv). The crude residue was purified by flash column chromatography (gradient of 0-20\% EtOAc/hexanes) to yield 2a as a white solid (18 mg, $0.135 \mathrm{mmol}, 34 \%$ ). Exposing this substrate to identical conditions in the absence of sodium bromide gave no yield of $\mathbf{2 a}$, as determined by GC-MS. The analytical data matched that of the authentic sample of $\mathbf{2 a}$ prepared independently (see Section G for independent synthesis).<smiles>Cc1cc(C)c(C#N)c([N+](=O)[O-])c1</smiles> 
2,4,6-Trimethylbenzonitrile (from ArBr) (12d) (CAS: 2571-52-0): According to General Procedure B, 12d was prepared using the following amounts of reagent: $\mathrm{NiBr}_{2}(\mathrm{bpy}) \cdot \mathrm{xH}_{2} \mathrm{O}(30$ $\mathrm{mg}, 0.080 \mathrm{mmol}, 20 \mathrm{~mol} \%$ ), $\mathrm{Zn}(0)$ dust ( $52 \mathrm{mg}, 0.80 \mathrm{mmol}, 2.0$ equiv), 2-bromomesitylene (61 $\mu \mathrm{L}, 0.40 \mathrm{mmol}, 1.0$ equiv), sodium bromide ( $82 \mathrm{mg}, 0.80 \mathrm{mmol}, 2.0$ equiv), DMA ( $1.0 \mathrm{~mL}$ ), and MPMN (1.0 mL of a $0.40 \mathrm{M}$ stock solution in DMA, $0.40 \mathrm{mmol}, 1.0$ equiv). The crude residue was purified by flash column chromatography (gradient of $0-20 \%$ EtOAc/hexanes) to yield 12d as a colourless oil (12 $\mathrm{mg}, 0.083 \mathrm{mmol}, 21 \%)$. Exposing this substrate to identical conditions in the absence of sodium bromide gave $<10 \%$ conversion of $\mathrm{ArBr}$, as determined by GC-MS.

Analytical data: ${ }^{16}{ }^{1} \mathbf{H}$ NMR $\left(400 \mathrm{MHz}, \mathrm{CDCl}_{3}, 298 \mathrm{~K}\right): \delta_{\mathrm{H}} 6.93(\mathrm{~s}, 2 \mathrm{H}), 2.48(\mathrm{~s}, 6 \mathrm{H}), 2.32(\mathrm{~s}, 3 \mathrm{H})$ ppm; ${ }^{13} \mathbf{C}$ NMR $\left(100 \mathrm{MHz}, \mathrm{CDCl}_{3}, 298 \mathrm{~K}\right): \delta_{\mathrm{C}} 142.9,142.1,128.3,117.7,110.4,21.7,20.7 \mathrm{ppm}$; $\mathbf{R}_{\mathbf{f}}\left(9: 1\right.$ hexanes/EtOAc; $\left.\mathrm{UV} / \mathrm{KMnO}_{4}\right): 0.62$. 


\section{F. Preparation of starting materials}

F.1. Synthesis of 2-methyl-2-phenylmalononitrile (MPMN)

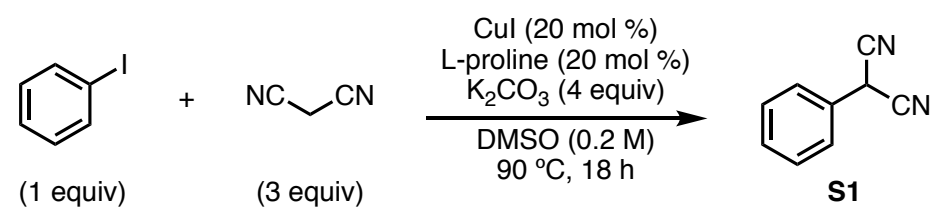

2-Phenylmalononitrile (S1) (CAS: 3041-40-5): ${ }^{38}$ To a flame-dried 500-mL flask were added copper(I) iodide (1.9 g, $10 \mathrm{mmol}, 20 \mathrm{~mol} \%$ ), L-proline (1.2 g, $10 \mathrm{mmol}, 20 \mathrm{~mol} \%$ ), potassium carbonate ( $28 \mathrm{~g}, 0.20 \mathrm{~mol}, 4.0$ equiv), and malononitrile $(9.9 \mathrm{~g}, 0.15 \mathrm{mmol}, 3.0$ equiv). The flask was sealed, evacuated and backfilled with $\mathrm{N}_{2}(\times 3)$, and DMSO was added $(250 \mathrm{~mL}, 0.20 \mathrm{M})$. Iodobenzene $\left(5.6 \mathrm{~mL}, 50 \mathrm{mmol}, 1.0\right.$ equiv) was added and the reaction was stirred at $90{ }^{\circ} \mathrm{C}$ for $18 \mathrm{~h}$. The flask was cooled to $0{ }^{\circ} \mathrm{C}$, opened to air, and $1 \mathrm{M} \mathrm{HCl}$ was added to bring the solution to $\mathrm{pH} 2-3$. The solution was extracted with EtOAc $(\times 3)$ and the organic fractions were combined, washed with brine $(\times 3)$, dried over $\mathrm{MgSO}_{4}$, and concentrated. The crude residue was purified by flash column chromatography (gradient of 10-20\% EtOAc/hexanes) to yield $\mathbf{S 1}$ as an off-white

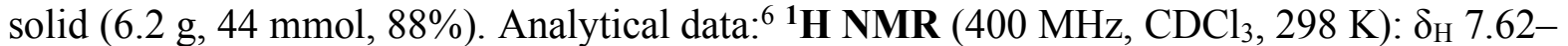
$7.42(\mathrm{~m}, 5 \mathrm{H}), 5.07$ (s, 1H) ppm; ${ }^{13} \mathbf{C}$ NMR (100 MHz, $\left.\mathrm{CDCl}_{3}, 298 \mathrm{~K}\right): \delta_{\mathrm{C}} 130.4,130.2,127.2$, 126.2, 111.7, 28.1 ppm; $\mathbf{R}_{\mathbf{f}}\left(9: 1\right.$ hexanes/EtOAc; $\left.\mathrm{KMnO}_{4} / \mathrm{PMA}\right): 0.16$.

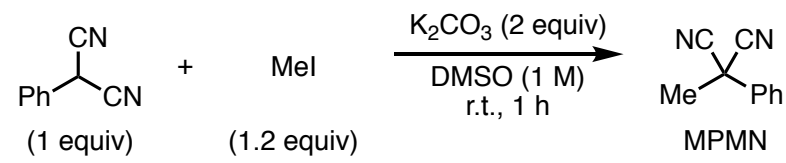

2-Methyl-2-phenylmalononitrile (MPMN) (CAS: 86164-70-7): To a 100-mL flask was added 2-phenylmalononitrile S1 (6.0 g, $42 \mathrm{mmol}, 1.0$ equiv). The flask was evacuated and backfilled with $\mathrm{N}_{2}(\times 3)$ and DMSO $(40 \mathrm{~mL}, 1.0 \mathrm{M}$; reagent grade) was added. Iodomethane $(3.1 \mathrm{~mL}, 50$ mmol, 1.2 equiv) was added followed by potassium carbonate ( $12 \mathrm{~g}, 84 \mathrm{mmol}, 2.0$ equiv). The reaction was stirred at r.t. for $2 \mathrm{~h}$. The reaction was quenched with sat. aq. $\mathrm{NH}_{4} \mathrm{Cl}$ and extracted with EtOAc $(\times 3)$. The organic fractions were combined, washed with brine $(\times 3)$, dried over $\mathrm{MgSO}_{4}$ and concentrated. The crude residue was purified by flash column chromatography (gradient of 5-20\% EtOAc/hexanes) to yield 1a as a colourless oil $(6.4 \mathrm{~g}, 41 \mathrm{mmol}, 98 \%)$. Analytical data: ${ }^{1} \mathbf{H}$ NMR $\left(400 \mathrm{MHz}, \mathrm{CDCl}_{3}, 298 \mathrm{~K}\right): \delta_{\mathrm{H}} 7.64-7.42(\mathrm{~m}, 5 \mathrm{H}), 2.13(\mathrm{~s}, 3 \mathrm{H}) \mathrm{ppm}$; ${ }^{13} \mathbf{C}$ NMR $\left(100 \mathrm{MHz}, \mathrm{CDCl}_{3}, 298 \mathrm{~K}\right): \delta_{\mathrm{C}} 113.1,130.0,129.8,129.3,115.7,36.4,29.5 \mathrm{ppm} ; \mathbf{R}_{\mathbf{f}}$ (9:1 hexanes/EtOAc; UV): 0.39. 


\section{F.2. Synthesis of aryl bromides}

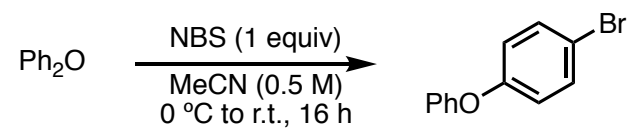

1-Bromo-4-phenoxybenzene (S2) (CAS: 101-55-3): ${ }^{39}$ To a $100-\mathrm{mL}$ flask with a stir bar were added diphenyl ether $(5.11 \mathrm{~g}, 30.0 \mathrm{mmol}, 1.00$ equiv) and $\mathrm{MeCN}(60 \mathrm{~mL}, 0.50 \mathrm{M})$ and the solution was cooled to $0{ }^{\circ} \mathrm{C}$. NBS (5.34 g, $30.0 \mathrm{mmol}, 1.00$ equiv) was added at once and the reaction was stirred for $16 \mathrm{~h}$ while being allowed to warm to r.t. The reaction was quenched with $\mathrm{H}_{2} \mathrm{O}$ and extracted with EtOAc $(\times 3)$. The organic fractions were combined, washed with sat. aq. $\mathrm{NaHCO}_{3}(\times 1), \mathrm{H}_{2} \mathrm{O}(\times 1)$, and brine $(\times 1)$, and were dried over $\mathrm{MgSO}_{4}$ and concentrated to yield 1-bromo-4-phenoxybenzene as a colourless oil (7.2 g, 29 mmol, 97\%). Analytical data: ${ }^{40}{ }^{1} \mathbf{H}$ NMR (400 MHz, $\left.\mathrm{CDCl}_{3}, 298 \mathrm{~K}\right): \delta_{\mathrm{H}} 7.48-7.40(\mathrm{~m}, 2 \mathrm{H}), 7.39-7.30(\mathrm{~m}, 2 \mathrm{H}), 7.17-7.07(\mathrm{~m}, 1 \mathrm{H})$, 7.05-6.97 (m, 2H), 6.93-6.85 (m, 2H) ppm; $\left.{ }^{13} \mathbf{C ~ N M R ~ ( 1 0 0 ~ M H z , ~} \mathrm{CDCl}_{3}, 298 \mathrm{~K}\right): \delta_{\mathrm{C}} 156.9$, $156.7,132.8,130.0,123.9,120.6,119.2,115.7 \mathrm{ppm}$.



(1.1 equiv)

1-Bromo-4-(((1R,2S,5R)-2-isopropyl-5-methylcyclohexyl)oxy)benzene (S3): To a 50-mL flask with a stir bar were added L-menthol (1.7 g, $11 \mathrm{mmol}, 1.1$ equiv), DMF (20 mL, $0.50 \mathrm{M})$ and sodium hydride ( $0.48 \mathrm{~g}$ of a $60 \% \mathrm{w} / \mathrm{w}$ dispersion in mineral oil, $12 \mathrm{mmol}, 1.2$ equiv), and the reaction was stirred at r.t. for $15 \mathrm{~min}$. 1-Bromo-4-fluorobenzene (1.1 mL, $10 \mathrm{mmol}, 1.0$ equiv) was added and the reaction was stirred at r.t. for $16 \mathrm{~h}$. The reaction was quenched with sat. aq. $\mathrm{NH}_{4} \mathrm{Cl}$ and extracted with EtOAc $(\times 3)$. The organic fractions were combined, washed with brine $(\times 1)$, dried over $\mathrm{MgSO}_{4}$, and concentrated. The crude residue was purified by flash column chromatography (gradient of 0-5\% EtOAc/hexanes) to yield the product as a colourless oil (2.2 g, $7.1 \mathrm{mmol}, 71 \%)$. Analytical data: ${ }^{1} \mathbf{H}$ NMR (400 MHz, $\left.\mathrm{CDCl}_{3}, 298 \mathrm{~K}\right): \delta_{\mathrm{H}} 7.38-7.30(\mathrm{~m}, 2 \mathrm{H})$, 6.81-6.72 (m, 2H), 3.97 (td, $J=10.6,4.2 \mathrm{~Hz}, 1 \mathrm{H}), 2.23-2.06(\mathrm{~m}, 2 \mathrm{H}), 1.76-1.66(\mathrm{~m}, 2 \mathrm{H}), 1.56-$ $1.38(\mathrm{~m}, 2 \mathrm{H}), 1.15-0.81(\mathrm{~m}, 9 \mathrm{H}), 0.76(\mathrm{~d}, J=7.0 \mathrm{~Hz}, 3 \mathrm{H}) \mathrm{ppm} ;{ }^{13} \mathbf{C} \mathbf{N M R}\left(100 \mathrm{MHz}, \mathrm{CDCl}_{3}\right.$, $298 \mathrm{~K}): \delta_{\mathrm{C}} 157.7,132.4,117.7,112.5,78.1,48.2,40.3,34.6,31.6,26.3,23.9,22.3,20.8 \mathrm{ppm}$; HRMS $m / z$ (DART): calcd for $\mathrm{C}_{16} \mathrm{H}_{23} \mathrm{BrO}(\mathrm{M}): 310.0932$; found 310.0934; IR (neat): 3454 , 2931, 1625, 1489, 1415, 1398, 1014, $823 \mathrm{~cm}^{-1}$; $\mathbf{R}_{\mathbf{f}}($ 5\% EtOAc/hexanes; UV/KMnO 4$): 0.71$.

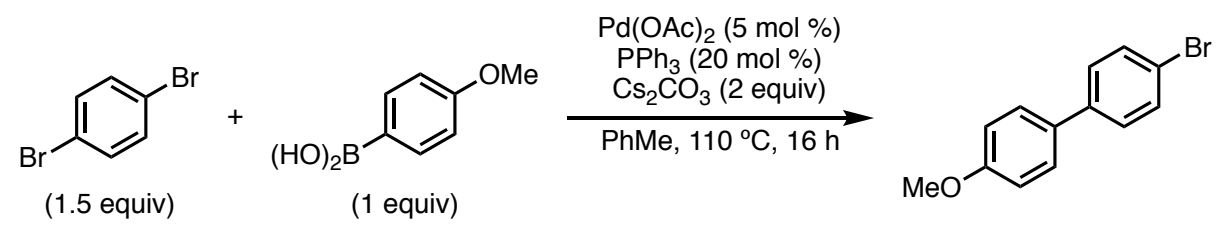

4-Bromo-4'-methoxy-1,1'-biphenyl (S4) (CAS: 58743-83-2): To a 50-mL flask with a stir bar were added 1,4-dibromobenzene ( $3.5 \mathrm{~g}, 15 \mathrm{mmol}, 1.5$ equiv), (4-methoxyphenyl)boronic acid ( $1.5 \mathrm{~g}, 10 \mathrm{mmol}, 1.0$ equiv), palladium(II) acetate $(0.11 \mathrm{~g}, 0.50 \mathrm{mmol}, 5.0 \mathrm{~mol} \%)$, triphenylphosphine $(0.52 \mathrm{~g}, 2.0 \mathrm{mmol}, 20 \mathrm{~mol} \%)$, cesium carbonate $(6.5 \mathrm{~g}, 20 \mathrm{mmol}, 2.0$ equiv), and $\mathrm{PhMe}(15 \mathrm{~mL}, 0.67 \mathrm{M})$. The reaction was stirred at $110^{\circ} \mathrm{C}$ for $16 \mathrm{~h}$. The reaction was cooled to r.t., quenched with sat. aq. $\mathrm{NH}_{4} \mathrm{Cl}$, and extracted with EtOAc $(\times 3)$. The organic fractions were 
combined, washed with brine $(\times 1)$, dried over $\mathrm{MgSO}_{4}$, and concentrated. The crude residue was purified by flash column chromatography (gradient of 0-5\% EtOAc/hexanes) to yield 4-bromo4'-methoxy-1,1'-biphenyl as a white solid (0.90 g, $3.4 \mathrm{mmol}, 34 \%)$. Analytical Data:41 $\mathbf{1}$ H NMR $\left(500 \mathrm{MHz}, \mathrm{CDCl}_{3}, 298 \mathrm{~K}\right): \delta_{\mathrm{H}} 7.58-7.46(\mathrm{~m}, 4 \mathrm{H}), 7.45-7.39(\mathrm{~m}, 2 \mathrm{H}), 7.01-6.95(\mathrm{~m}, 2 \mathrm{H}), 3.85$ (s, 3H) ppm; ${ }^{13} \mathbf{C}$ NMR (125 MHz, $\left.\mathrm{CDCl}_{3}, 298 \mathrm{~K}\right): \delta_{\mathrm{C}} 159.6,139.9,132.6,131.9,128.4,128.1$, 120.9, 114.5, 55.5 ppm; $\mathbf{R}_{\mathbf{f}}(5 \%$ EtOAc/hexanes; UV): 0.52.

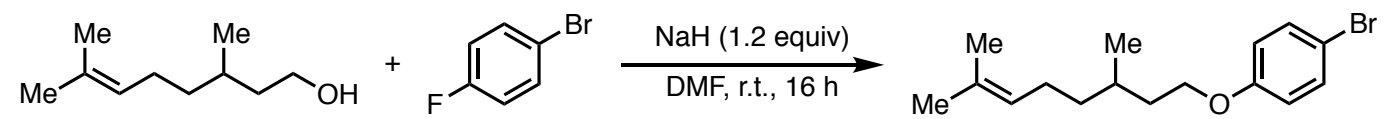

$$
\text { (1.1 equiv) (1 equiv) }
$$

1-Bromo-4-((3,7-dimethyloct-6-en-1-yl)oxy)benzene (S5): To a 50-mL flask with a stir bar were added DMF $(20 \mathrm{~mL}, 0.50 \mathrm{M})$, citronellol $(2.0 \mathrm{~mL}, 11 \mathrm{mmol}, 1.1$ equiv) and $\mathrm{NaH}(0.48 \mathrm{~g}$ of a $60 \% \mathrm{w} / \mathrm{w}$ dispersion in mineral oil, $12 \mathrm{mmol}, 1.2$ equiv). The reaction was stirred at r.t. for 15 min. 1-Bromo-4-fluorobenzene ( $1.1 \mathrm{~mL}, 10 \mathrm{mmol}, 1.0$ equiv) was added and the reaction was stirred at r.t. for $16 \mathrm{~h}$. The reaction was quenched with sat. aq. $\mathrm{NH}_{4} \mathrm{Cl}$ and extracted with EtOAc $(\times 3)$. The organic fractions were combined, washed with brine $(\times 1)$, dried over $\mathrm{MgSO}_{4}$, and concentrated. The crude residue was purified by flash column chromatography (gradient of 0 $2 \% \mathrm{EtOAc/hexanes)}$ to yield the product as a colourless oil (0.69 g, $2.2 \mathrm{mmol}, 22 \%)$. Analytical data: ${ }^{1} \mathbf{H}$ NMR (400 MHz, $\left.\mathrm{CDCl}_{3}, 298 \mathrm{~K}\right)$ : 7.39-7.32 (m, 2H), 6.81-6.73 (m, 2H), 5.14-5.06 (m, $1 \mathrm{H}), 4.03-3.89(\mathrm{~m}, 2 \mathrm{H}), 2.10-1.90(\mathrm{~m}, 2 \mathrm{H}), 1.88-1.75(\mathrm{~m}, 1 \mathrm{H}), 1.73-1.51(\mathrm{~m}, 8 \mathrm{H}), 1.45-1.33$ $(\mathrm{m}, 1 \mathrm{H}), 1.28-1.16(\mathrm{~m}, 1 \mathrm{H}), 0.95(\mathrm{~d}, J=6.5 \mathrm{~Hz}, 3 \mathrm{H}) \mathrm{ppm} ;{ }^{13} \mathbf{C} \mathbf{N M R}\left(100 \mathrm{MHz}, \mathrm{CDCl}_{3}, 298 \mathrm{~K}\right)$ : $\delta_{\mathrm{C}} 158.3,132.2,131.4,124.6,116.3,112.6,66.6,37.1,36.0,29.5,25.7,25.5,19.6,17.7 \mathrm{ppm}$; HRMS $m / z$ (DART): calcd for $\mathrm{C}_{16} \mathrm{H}_{24} \mathrm{BrO}(\mathrm{M}+\mathrm{H}): 311.1011$; found 311.0992; IR (neat): 2954, 2924, 2869, 1588, 1485, 1240, 1235, 1014, 994, 820, $641 \mathrm{~cm}^{-1} ; \mathbf{R}_{\mathbf{f}}(5 \%$ EtOAc/hexanes; $\left.\mathrm{UV} / \mathrm{KMnO}_{4}\right): 0.65$.



4-Bromo-2-chloro-1-methoxybenzene (S6) (CAS: 50638-47-6): To a 25-mL flask with a stir bar were added 4-bromo-2-chlorophenol ( $0.21 \mathrm{~g}, 1.0 \mathrm{mmol}, 1.0$ equiv), potassium carbonate (0.68 g, $4.9 \mathrm{mmol}, 4.9$ equiv), DMF (5.2 mL, $0.19 \mathrm{M})$, and iodomethane (0.19 mL, $3.0 \mathrm{mmol}, 3.0$ equiv), and the reaction was stirred at $70{ }^{\circ} \mathrm{C}$ for $16 \mathrm{~h}$. The reaction was cooled to r.t., quenched with sat. aq. $\mathrm{NH}_{4} \mathrm{Cl}$, and extracted with EtOAc $(\times 3)$, and the organic fractions were combined, dried over $\mathrm{MgSO}_{4}$, and concentrated. The crude residue was purified by flash column chromatography (gradient of 1-5\% EtOAc/hexanes) to yield $\mathbf{S 6}$ as a white solid $(0.21 \mathrm{~g}, 0.94$ mmol, 94\%). Analytical Data: ${ }^{42} \mathbf{H}$ NMR (400 MHz, $\left.\mathrm{CDCl}_{3}, 298 \mathrm{~K}\right): 7.50$ (d, $\left.J=2.4 \mathrm{~Hz}, 1 \mathrm{H}\right)$, $7.33(\mathrm{dd}, J=8.8,2.4 \mathrm{~Hz}, 1 \mathrm{H}), 7.80(\mathrm{~d}, J=8.8 \mathrm{~Hz}, 1 \mathrm{H}), 3.88(\mathrm{~s}, 3 \mathrm{H}) \mathrm{ppm} ;{ }^{13} \mathbf{C} \mathbf{N M R}(100 \mathrm{MHz}$, $\left.\mathrm{CDCl}_{3}, 298 \mathrm{~K}\right): \delta_{\mathrm{C}} 154.8,133.1,131.0,124.1,113.8,113.0,56.8 \mathrm{ppm}$.

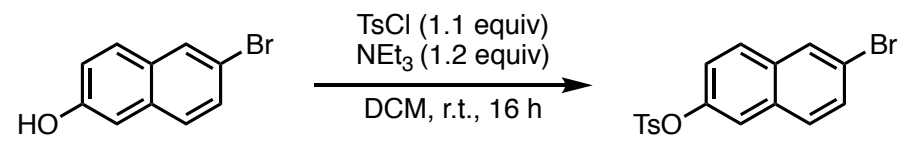

6-Bromonaphthalen-2-yl 4-methylbenzenesulfonate (S7): To a 50-mL flask were added 6bromonaphthol (1.1 g, $5.0 \mathrm{mmol}, 1.0$ equiv), $\mathrm{CH}_{2} \mathrm{Cl}_{2}(10 \mathrm{~mL}, 0.50 \mathrm{M})$, triethylamine $(0.84 \mathrm{~mL}$, $6.0 \mathrm{mmol}, 1.2$ equiv), and tosyl chloride ( $1.0 \mathrm{~g}, 5.5 \mathrm{mmol}, 1.1$ equiv). The reaction was stirred at 
r.t. for $16 \mathrm{~h}$. The solution was concentrated and the crude material was purified by flash column chromatography (gradient of 0-20\% EtOAc/hexanes) to yield the product as a white solid (1.4 g, 3.7 mmol, 74\%). Analytical data: ${ }^{1} \mathbf{H}$ NMR $\left(500 \mathrm{MHz}, \mathrm{CDCl}_{3}, 298 \mathrm{~K}\right): \delta_{\mathrm{H}} 7.99-7.96(\mathrm{~m}, 1 \mathrm{H})$, $7.74-7.70(\mathrm{~m}, 2 \mathrm{H}), 7.67-7.63(\mathrm{~m}, 1 \mathrm{H}), 7.63-7.60(\mathrm{~m}, 1 \mathrm{H}), 7.56(\mathrm{dd}, J=8.8,1.9 \mathrm{~Hz}, 1 \mathrm{H}), 7.47-$ $7.44(\mathrm{~m}, 1 \mathrm{H}), 7.32-7.28(\mathrm{~m}, 2 \mathrm{H}), 7.11(\mathrm{dd}, J=9.0,1.9 \mathrm{~Hz}, 1 \mathrm{H}), 2.45(\mathrm{~s}, 3 \mathrm{H}) \mathrm{ppm} ;{ }^{13} \mathbf{C}$ NMR $\left(125 \mathrm{MHz}, \mathrm{CDCl}_{3}, 298 \mathrm{~K}\right): \delta_{\mathrm{C}} 147.5,145.7,133.0,132.4,132.0,130.5,130.0,130.0,129.6$, 129.0, 128.7, 122.5, 120.5, 120.2, 21.9 ppm; HRMS $m / z$ (DART): calcd for $\mathrm{C}_{17} \mathrm{H}_{17} \mathrm{BrNO}_{3} \mathrm{~S}$ $\left(\mathrm{M}+\mathrm{NH}_{4}\right)$ 394.01125; found 394.0099; IR (neat): 3091, 1585, 1500, 1376, 1173, 1139, 1120 , 1092, 911, 887, 833, $808 \mathrm{~cm}^{-1}$; m.p.: $134-135^{\circ} \mathrm{C}$; $\mathbf{R}_{\mathbf{f}}$ (5\% EtOAc/hexanes; UV): 0.29.

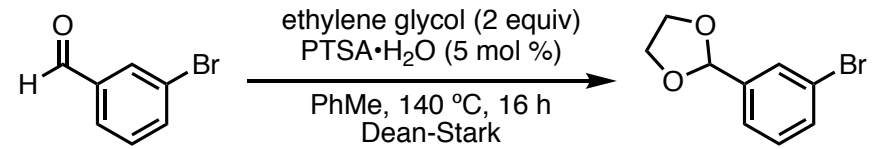

2-(3-Bromophenyl)-1,3-dioxolane (S8) (CAS: 17789-14-9): To a 50-mL flask with stir bar were added $p$-toluenesulfonic acid monohydrate $(0.19 \mathrm{~g}, 1.0 \mathrm{mmol}, 0.050$ equiv), PhMe $(20 \mathrm{~mL}$, $1.0 \mathrm{M})$, 3-bromobenzaldehyde $(2.3 \mathrm{~mL}, 20 \mathrm{mmol}, 1.0$ equiv), and ethylene glycol $(2.2 \mathrm{~mL}, 40$ mmol, 2.0 equiv). The flask was equipped with a Dean-Stark apparatus and was stirred at $140{ }^{\circ} \mathrm{C}$ for $16 \mathrm{~h}$. The reaction was cooled to r.t. and concentrated. The crude material was purified by flash column chromatography (gradient of $0-10 \%$ EtOAc/hexanes) to yield the product as a colourless oil (3.0 g, $13 \mathrm{mmol}, 65 \%)$. Analytical data: ${ }^{43}{ }^{1} \mathbf{H} \mathbf{~ N M R}\left(400 \mathrm{MHz}, \mathrm{CDCl}_{3}, 298 \mathrm{~K}\right): \delta_{\mathrm{H}}$ 7.70-7.63 (m, 1H), 7.56-7.48 (m, 1H), 7.47-7.38 (m, 1H), 7.32-7.21 (m, 1H), 5.81 (s, 1H), 4.19-4.00 (m, 4H) ppm; ${ }^{13} \mathbf{C}$ NMR (100 MHz, $\left.\mathrm{CDCl}_{3}, 298 \mathrm{~K}\right): \delta_{\mathrm{C}} 140.5,132.3,130.1,129.7$, 125.3, 122.6, 102.8, 65.4 ppm; $\mathbf{R}_{\mathbf{f}}(9: 1$ hexanes/EtOAc; UV/KMnO 4$): 0.40$.

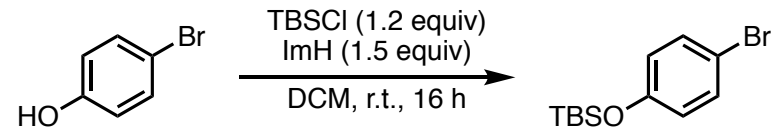

(4-Bromophenoxy)(tert-butyl)dimethylsilane (S9) (CAS: 67963-68-2): To a 100-mL flask with stir bar were added imidazole ( $1.2 \mathrm{~g}, 18 \mathrm{mmol}, 1.5$ equiv), TBSCl ( $2.1 \mathrm{~g}, 14 \mathrm{mmol}, 1.2$ equiv), 4-bromophenol (2.1 g, $12 \mathrm{mmol}, 1.0$ equiv), and $\mathrm{CH}_{2} \mathrm{Cl}_{2}(24 \mathrm{~mL}, 0.50 \mathrm{M})$. The reaction was stirred at r.t. for $16 \mathrm{~h}$. The reaction was concentrated and the crude material was purified by flash column chromatography (gradient of 0-5\% EtOAc/hexanes) to yield $\mathbf{S 9}$ as a colourless oil (3.4 g, $11.8 \mathrm{mmol}, 98 \%)$. Analytical data: ${ }^{44}{ }^{1} \mathbf{H}$ NMR (400 MHz, $\left.\mathrm{CDCl}_{3}, 298 \mathrm{~K}\right): \delta_{\mathrm{H}} 7.36-7.28$ (m, 2H), 6.76-6.68 (m, 2H), $0.98(\mathrm{~s}, 9 \mathrm{H}), 0.19$ (s, 6H) ppm; ${ }^{13} \mathbf{C}$ NMR (100 MHz, CDCl 3 , 298 $\mathrm{K}): \delta_{\mathrm{C}} 155.0,132.4,122.1,113.8,25.8,18.3,-4.3 \mathrm{ppm} ; \mathbf{R}_{\mathbf{f}}(9: 1$ hexanes/EtOAc; UV): 0.82.

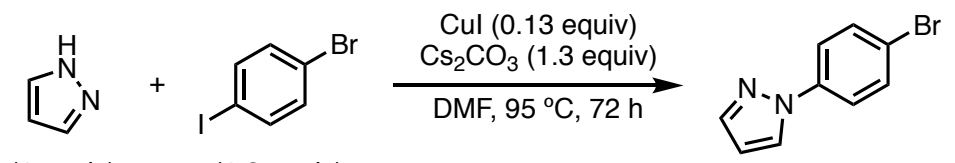

(1 equiv) (1.3 equiv)

1-(4-Bromophenyl)-1H-pyrazole (S10) (CAS: 13788-92-6): To a 10-mL flask with a stir bar were added pyrazole $(0.14 \mathrm{~g}, 2.1 \mathrm{mmol}, 1.0$ equiv), 1-iodo-4-bromobenzene $(0.79 \mathrm{~g}, 2.8 \mathrm{mmol}$, 1.3 equiv), copper(I) iodide (53 mg, $0.28 \mathrm{mmol}, 0.13$ equiv), cesium carbonate $(0.91 \mathrm{~g}, 2.8$ mmol, 1.3 equiv), and DMF $(2.9 \mathrm{~mL}, 1.4 \mathrm{M})$. The reaction was stirred at $95{ }^{\mathrm{a}} \mathrm{C}$ for $72 \mathrm{~h}$. The reaction was cooled to r.t., quenched with $\mathrm{H}_{2} \mathrm{O}$, and extracted with EtOAc $(\times 3)$, and the organic fractions were collected, washed with brine $(\times 1)$, dried over $\mathrm{MgSO}_{4}$, and concentrated. The residue was purified by flash column chromatography (8:2 hexanes/EtOAc) to yield $\mathbf{S 1 0}$ as a 
white solid (0.39 g, $1.7 \mathrm{mmol}, 81 \%$ ). Analytical data: ${ }^{45}{ }^{1} \mathbf{H}$ NMR (400 MHz, $\mathrm{CDCl}_{3}, 298 \mathrm{~K}$ ): 7.91-7.88 (m, 1H), 7.74-7.70 (m, 1H), 7.62-7.54 (m, 4H), 6.49-6.46 (m, 1H) ppm; ${ }^{13} \mathbf{C}$ NMR $\left(100 \mathrm{MHz}, \mathrm{CDCl}_{3}, 298 \mathrm{~K}\right): \delta_{\mathrm{C}} 141.6,139.4,132.6,126.8,120.8,119.8,108.2 \mathrm{ppm} ; \mathbf{R}_{\mathbf{f}}(8: 2$ hexanes/EtOAc; UV): 0.22.

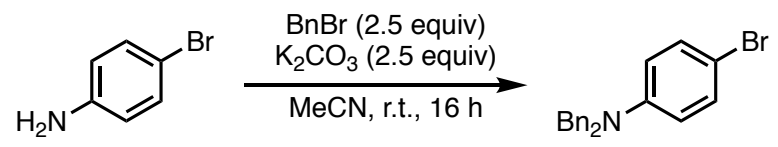

$\boldsymbol{N}, \boldsymbol{N}$-Dibenzyl-4-bromoaniline (S11) (CAS: 65145-14-4): To a 100-mL flask were added 4bromoaniline ( $3.4 \mathrm{~g}, 20 \mathrm{mmol}, 1.0$ equiv), potassium carbonate (6.9 g, $50 \mathrm{mmol}, 2.5$ equiv), $\mathrm{MeCN}(40 \mathrm{~mL}, 0.50 \mathrm{M})$, and benzyl bromide (5.2 $\mathrm{mL}, 44 \mathrm{mmol}, 2.2$ equiv). The reaction was stirred at r.t. for $16 \mathrm{~h}$. The reaction was diluted with EtOAc $(100 \mathrm{~mL})$ and filtered using a fritted funnel. The filter cake was extracted with EtOAc $(50 \mathrm{~mL})$. The organic filtrate and layers were combined and concentrated. The crude residue was purified by flash column chromatography (gradient of 0-60\% EtOAc/hexanes) to yield $\mathbf{S 1 1}$ as a white solid (2.5 g, $7.1 \mathrm{mmol}, 36 \%$ ). Analytical data: ${ }^{46}{ }^{1} \mathbf{H}$ NMR $\left(500 \mathrm{MHz}, \mathrm{CDCl}_{3}, 298 \mathrm{~K}\right): \delta_{\mathrm{H}} 7.40-7.20(\mathrm{~m}, 12 \mathrm{H}), 6.67-6.59(\mathrm{~m}$, 2H), 4.65 (s, 4H) ppm; ${ }^{13} \mathbf{C}$ NMR (125 MHz, $\left.\mathrm{CDCl}_{3}, 298 \mathrm{~K}\right): \delta_{\mathrm{C}} 148.2,138.1,132.0,128.9$, 127.2, 126.7, 114.3, 108.7, 54.6 ppm; Rf (5\% EtOAc/hexanes; UV): 0.56.



1-(4-Bromophenyl)ethan-1-ol (S12) (CAS: 5391-88-8): To a 250-mL Erlenmeyer flask with a stir bar were added 4'-bromoacetophenone (4.0 g, $20 \mathrm{mmol}, 1.0$ equiv), $\mathrm{MeOH}$ (60 mL, $0.33 \mathrm{M}$ ), and sodium borohydride ( $0.91 \mathrm{~g}, 24 \mathrm{mmol}, 1.2$ equiv), and the reaction was stirred at r.t. for $1 \mathrm{~h}$. The reaction was quenched with $1 \mathrm{M} \mathrm{HCl}$ and extracted with EtOAc $(\times 3)$ and the organic fractions were combined, washed with brine $(\times 1)$, dried over $\mathrm{MgSO}_{4}$, and concentrated to yield S12 as a colourless oil (4.0 g, $19.9 \mathrm{mmol}, 99 \%)$. Analytical data: ${ }^{47}{ }^{1} \mathbf{H} \mathbf{~ N M R}\left(400 \mathrm{MHz}, \mathrm{CDCl}_{3}\right.$, $298 \mathrm{~K}): 7.55-7.46(\mathrm{~m}, 2 \mathrm{H}), 7.33-7.25(\mathrm{~m}, 2 \mathrm{H}), 4.90(\mathrm{q}, J=6.4 \mathrm{~Hz}, 1 \mathrm{H}), 1.90$ (br s, 1H), 1.50 (d, $J=6.4 \mathrm{~Hz}, 3 \mathrm{H}) \mathrm{ppm} ;{ }^{13} \mathbf{C} \mathbf{N M R}\left(100 \mathrm{MHz}, \mathrm{CDCl}_{3}, 298 \mathrm{~K}\right): \delta_{\mathrm{C}} 144.9,131.7,127.3,121.3,69.9$, $25.4 \mathrm{ppm}$.

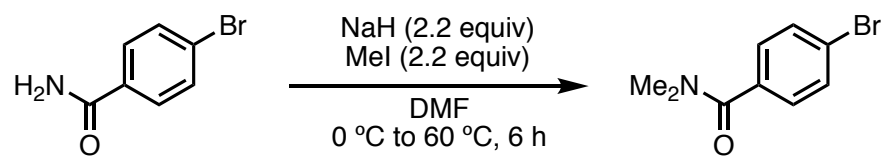

4-Bromo- $\boldsymbol{N}, \boldsymbol{N}$-dimethylbenzamide (S13) (CAS: 18469-37-9): To a 50-mL round bottom flask with a stir bar were added 4-bromobenzamide ( $0.40 \mathrm{~g}, 2.0 \mathrm{mmol}, 1.0$ equiv) and DMF ( $10 \mathrm{~mL}$, $0.20 \mathrm{M})$. The solution was cooled to $0^{\circ} \mathrm{C}$ and sodium hydride $(0.18 \mathrm{~g}$ of a $60 \% \mathrm{w} / \mathrm{w}$ dispersion in mineral oil, $4.4 \mathrm{mmol}, 1.1$ equiv) was added, and the reaction was stirred at $0{ }^{\circ} \mathrm{C}$ for $10 \mathrm{~min}$. Iodomethane $\left(0.28 \mathrm{~mL}, 4.4 \mathrm{mmol}, 2.2\right.$ equiv) was added, and the reaction was stirred at $60{ }^{\circ} \mathrm{C}$ for $6 \mathrm{~h}$. The reaction mixture was cooled to room temperature, quenched with $\mathrm{H}_{2} \mathrm{O}$, and extracted with EtOAc $(\times 3)$. The organic layers were combined, washed with brine $(\times 1)$, dried over $\mathrm{MgSO}_{4}$, and concentrated. The crude residue was purified by flash column chromatography (50\% EtOAc/hexanes) to yield $\mathbf{S 1 3}$ as a white solid (0.41 g, $1.8 \mathrm{mmol}, 90 \%)$. Analytical data: ${ }^{48}$ ${ }^{1} \mathbf{H}$ NMR $\left(400 \mathrm{MHz}, \mathrm{CDCl}_{3}, 298 \mathrm{~K}\right): \delta_{\mathrm{H}} 7.58-7.50(\mathrm{~m}, 2 \mathrm{H}), 7.34-7.27$ (m, 2H), 3.10 (s, 3H), 
2.97 (s, 3H) ppm; ${ }^{13} \mathbf{C} \mathbf{N M R}\left(100 \mathrm{MHz}, \mathrm{CDCl}_{3}, 298 \mathrm{~K}\right): \delta_{\mathrm{C}} 170.7,135.3,131.7,128.9,123.9$, 39.6, 35.5 ppm; $\mathbf{R}_{\mathbf{f}}(50 \%$ EtOAc/hexanes; UV): 0.22 .

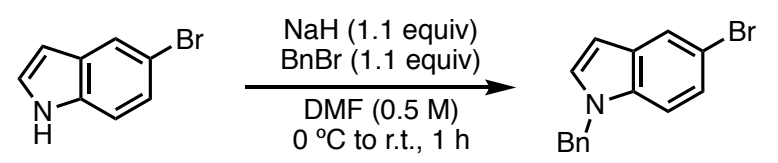

1-Benzyl-5-bromo-1H-indole (S14) (CAS: 10075-51-1): To a flame-dried 100-mL flask were added 5-bromoindole (2.9 g, $15 \mathrm{mmol}, 1.0$ equiv) and DMF (30 mL, $0.50 \mathrm{M})$. The solution was cooled to $0{ }^{\circ} \mathrm{C}$ and sodium hydride $(0.68 \mathrm{~g}, 17 \mathrm{mmol}, 1.1$ equiv) was added. The reaction was stirred at $0{ }^{\circ} \mathrm{C}$ for $15 \mathrm{~min}$. Then, benzyl bromide $(2.0 \mathrm{~mL}, 17 \mathrm{mmol}, 1.1$ equiv) was added and the reaction was stirred at r.t. for $1 \mathrm{~h}$. The reaction was quenched with sat. aq. $\mathrm{NH}_{4} \mathrm{Cl}$ and extracted with EtOAc $(\times 3)$. The organic fractions were combined, washed with brine $(\times 1)$, and concentrated. The crude residue was purified by flash column chromatography (gradient of 0 $20 \% \mathrm{EtOAc} /$ hexanes) to yield $\mathbf{S 1 4}$ as a white solid (3.4 g, $12 \mathrm{mmol}, 80 \%)$. Analytical data: ${ }^{49}{ }^{1} \mathbf{H}$ NMR (400 MHz, $\left.\mathrm{CDCl}_{3}, 298 \mathrm{~K}\right): \delta_{\mathrm{H}} 7.83-7.77(\mathrm{~m}, 1 \mathrm{H}), 7.38-7.22(\mathrm{~m}, 4 \mathrm{H}), 7.20-7.06(\mathrm{~m}, 4 \mathrm{H})$, 6.55-6.49 (m, 1H), $5.33(\mathrm{~s}, 2 \mathrm{H}) \mathrm{ppm} ;{ }^{13} \mathbf{C}$ NMR $\left(100 \mathrm{MHz}, \mathrm{CDCl}_{3}, 298 \mathrm{~K}\right): \delta_{\mathrm{C}} 137.1,135.0$, $130.4,129.5,128.9,127.8,126.7,124.6,123.5,112.9,111.2,101.3,50.3 \mathrm{ppm} ; \mathbf{R}_{\mathbf{f}}(9: 1$ hexanes/EtOAc; UV): 0.52 .

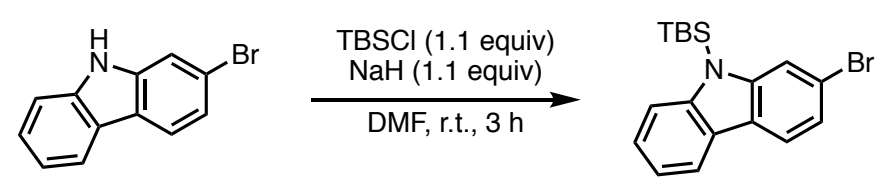

2-Bromo-9-(tert-butyldimethylsilyl)-9H-carbazole (S15): To a flame-dried 50-mL flask with a stir bar were added 2-bromo-9H-carbazole $(0.74 \mathrm{~g}, 3.0 \mathrm{mmol}, 1.0$ equiv), DMF $(10 \mathrm{~mL}, 0.30 \mathrm{M})$, and sodium hydride $(0.13 \mathrm{~g}, 3.3 \mathrm{mmol}, 1.1$ equiv). The reaction was stirred at r.t. for $15 \mathrm{~min}$, TBSCl ( $0.50 \mathrm{~g}, 3.3 \mathrm{mmol}, 1.1$ equiv) was added and the reaction was stirred at r.t. for $3 \mathrm{~h}$. The reaction was quenched with sat. aq. $\mathrm{NH}_{4} \mathrm{Cl}$ and extracted with EtOAc $(\times 3)$. The organic fractions were combined, washed with brine $(\times 1)$, dried over $\mathrm{MgSO}_{4}$, and concentrated. The crude residue was purified by flash column chromatography (gradient of 0-5\% EtOAc/hexanes) to yield $\mathbf{S 1 5}$ as an off-white solid $(0.76 \mathrm{~g}, 2.1 \mathrm{mmol}, 70 \%)$. Analytical data: ${ }^{1} \mathbf{H}$ NMR $\left(400 \mathrm{MHz}, \mathrm{CDCl}_{3}, 298\right.$ $\mathrm{K}): \delta_{\mathrm{H}} 8.07-7.98(\mathrm{~m}, 1 \mathrm{H}), 7.96-7.86(\mathrm{~m}, 1 \mathrm{H}), 7.80-7.70(\mathrm{~m}, 1 \mathrm{H}), 7.65-7.54(\mathrm{~m}, 1 \mathrm{H}), 7.44-7.30$ (m, 2H), 7.29-7.19 (m, 1H), 1.05 (s, 9H), $0.76(\mathrm{~s}, 6 \mathrm{H}) \mathrm{ppm} ;{ }^{13} \mathbf{C}$ NMR (100 MHz, $\mathrm{CDCl}_{3}, 298$ $\mathrm{K}): \delta_{\mathrm{C}} 146.0,145.2,125.7,125.7,125.3,122.8,120.8,120.0,119.8,118.8,117.0,114.2,26.6$, 20.6, $-1.2 \mathrm{ppm}$; HRMS $\mathrm{m} / z$ (DART): calcd for $\mathrm{C}_{18} \mathrm{H}_{23} \mathrm{BrNSi}(\mathrm{M}+\mathrm{H}$ ): 360.0783 ; found 360.0785; IR (neat): 2928, 2857, 1588, 1458, 1453, 1414, 1246, 964, 820, 807, 749, $725 \mathrm{~cm}^{-1}$; m.p.: 144$147^{\circ} \mathrm{C} ; \mathbf{R}_{\mathbf{f}}(9: 1$ hexanes/EtOAc; UV): 0.80 .

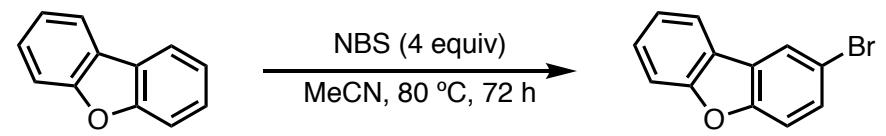

2-Bromodibenzofuran (S16) (CAS: 86-76-0): ${ }^{39}$ To a 100-mL flask were added dibenzofuran ( $2.4 \mathrm{~g}, 14 \mathrm{mmol}, 1.0$ equiv), $\mathrm{MeCN}$ (14 mL, $1.0 \mathrm{M}$ ), and NBS (10 g, $56 \mathrm{mmol}, 4.0$ equiv). The reaction was stirred at $80{ }^{\circ} \mathrm{C}$ for $72 \mathrm{~h}$. The reaction was cooled to r.t., quenched with $\mathrm{H}_{2} \mathrm{O}$, and extracted with EtOAc $(\times 3)$. The organic fractions were combined, washed with brine $(\times 1)$, dried over $\mathrm{MgSO}_{4}$, and concentrated. The crude residue was purified by flash column chromatography (gradient of 0-10\% EtOAc/hexanes) to yield $\mathbf{S 1 6}$ as an off-white solid, which contained some 
unbrominated dibenzofuran (2.5 g, $10 \mathrm{mmol}, 71 \%)$. Analytical data: ${ }^{50}{ }^{1} \mathbf{H}$ NMR (400 MHz, $\left.\mathrm{CDCl}_{3}, 298 \mathrm{~K}\right): \delta_{\mathrm{H}} 8.10-8.05(\mathrm{~m}, 1 \mathrm{H}), 7.94-7.88(\mathrm{~m}, 1 \mathrm{H}), 7.62-7.41(\mathrm{~m}, 4 \mathrm{H}), 7.40-7.32(\mathrm{~m}, 1 \mathrm{H})$ ppm; ${ }^{13} \mathbf{C}$ NMR $\left(100 \mathrm{MHz}, \mathrm{CDCl}_{3}, 298 \mathrm{~K}\right): \delta_{\mathrm{C}} 156.8,155.1,130.0,128.1,126.4,123.7,123.3$, 123.2, 121.0, 115.7, 113.3, 112.0 ppm; $\mathbf{R}_{\mathbf{f}}(5 \%$ EtOAc/hexanes; UV): 0.75.



Benzo[d][1,3]dioxol-5-yl(5-bromothiophen-2-yl)methanol (S17): To a flame-dried 50-mL flask with a stir bar were added 2,5-dibromothiophene $(0.90 \mathrm{~mL}, 8.0 \mathrm{mmol}, 1.0$ equiv) and THF $(16 \mathrm{~mL}, 0.50 \mathrm{M})$. The solution was cooled to $-78^{\circ} \mathrm{C}$. $n$-Butyllithium $(5.5 \mathrm{~mL}$ of a $1.6 \mathrm{M}$ solution in hexanes, $8.8 \mathrm{mmol}, 1.1$ equiv) was added dropwise and the reaction was stirred at $-78^{\circ} \mathrm{C}$ for $1 \mathrm{~h}$. Piperonal (1.4 g, $9.6 \mathrm{mmol}, 1.2$ equiv) was added at once and the reaction was stirred at $-78{ }^{\circ} \mathrm{C}$ for $1 \mathrm{~h}$. The reaction was warmed to r.t., quenched with sat. aq. $\mathrm{NH}_{4} \mathrm{Cl}$, and extracted with EtOAc $(\times 3)$. The organic fractions were combined, washed with brine $(\times 1)$, dried over $\mathrm{MgSO}_{4}$, and concentrated. The crude residue was purified by flash column chromatography (gradient of 0-30\% EtOAc/hexanes). The fractions containing product were collected and concentrated to yield $2.0 \mathrm{~g}$ of a crude material (ca. $6.4 \mathrm{mmol}$ ), which was used in the next step without further purification.

5-((5-Bromothiophen-2-yl)(methoxy)methyl)benzo[d][1,3]dioxole (S18): To a 50-mL flask with a stir bar were added S17 (2.0 g, $6.4 \mathrm{mmol}, 1.0$ equiv) and THF (21 mL, $0.30 \mathrm{M})$. The solution was cooled to $0{ }^{\circ} \mathrm{C}$. Sodium hydride $(0.28 \mathrm{~g}$ of a $60 \% \mathrm{w} / \mathrm{w}$ dispersion in mineral oil, 7.0 mmol, 1.1 equiv) was added and the reaction was stirred at $0{ }^{\circ} \mathrm{C}$ for $15 \mathrm{~min}$. Iodomethane $(0.44$ $\mathrm{mL}, 7.0 \mathrm{mmol}, 1.1$ equiv) was added and the reaction was stirred at r.t. for $1 \mathrm{~h}$. The reaction was quenched with sat. aq. $\mathrm{NH}_{4} \mathrm{Cl}$ and extracted with EtOAc $(\times 3)$. The organic fractions were combined, washed with brine $(\times 1)$, dried over $\mathrm{MgSO}_{4}$, and concentrated. The crude residue was purified by flash column chromatography to yield $\mathbf{S 1 8}$ as an orange semisolid (1.5 g, $4.6 \mathrm{mmol}$, $58 \%$ over two steps). Analytical data: ${ }^{1} \mathrm{H}$ NMR $\left(400 \mathrm{MHz}, \mathrm{CDCl}_{3}, 298 \mathrm{~K}\right): \delta_{\mathrm{H}} 6.89-6.76(\mathrm{~m}$, 4H), 6.57-6.53 (m, 1H), 5.98-5.93 (m, 2H), 5.26 (br s, 1H), 3.36 (br s, 3H) ppm; ${ }^{13}$ C NMR $(100$ $\left.\mathrm{MHz}, \mathrm{CDCl}_{3}, 298 \mathrm{~K}\right): 148.3,148.1,147.7,134.6,129.3,125.4,120.8,112.5,108.2$, 107.3, 101.3, 81.4, 56.9 ppm; IR (neat): 2988, 2929, 2871, 2822, 1502, 1487, 1440, 1239, 1095, 1077, 1038, 968, 929, $781 \mathrm{~cm}^{-1}$; $\mathbf{R}_{\mathbf{f}}(9: 1$ hexanes/EtOAc; UV): 0.60 .

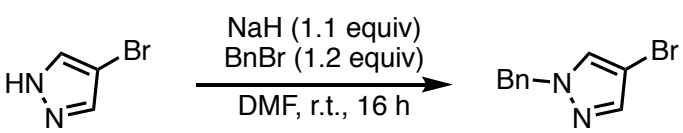

1-Benzyl-4-bromo-1H-pyrazole (S19) (CAS: 50877-41-3): To a 50-mL flask with a stir bar were added 4-bromopyrazole ( $0.73 \mathrm{~g}, 5.0 \mathrm{mmol}, 1.0$ equiv), DMF (15 mL, $0.33 \mathrm{M})$, and sodium hydride ( $0.22 \mathrm{~g}$ of a $60 \% \mathrm{w} / \mathrm{w}$ dispersion in mineral oil, $5.5 \mathrm{mmol}, 1.1$ equiv). The reaction was stirred at r.t. for $15 \mathrm{~min}$. Benzyl bromide $(0.71 \mathrm{~mL}, 6.0 \mathrm{mmol}, 1.2$ equiv) was added and the reaction was stirred at r.t. for $16 \mathrm{~h}$. The reaction was quenched with $\mathrm{H}_{2} \mathrm{O}$ and extracted with EtOAc $(\times 3)$. The organic fractions were combined, washed with brine $(\times 1)$, dried over $\mathrm{MgSO}_{4}$, and concentrated. The crude residue was purified by flash column chromatography (gradient of $0-10 \% \mathrm{EtOAc} / \mathrm{hexanes}$ ) to yield $\mathbf{S 1 9}$ as a colourless oil (1.1 g, $4.6 \mathrm{mmol}, 92 \%)$. Analytical 
data: ${ }^{51}{ }^{1} \mathbf{H}$ NMR $\left(500 \mathrm{MHz}, \mathrm{CDCl}_{3}, 298 \mathrm{~K}\right): \delta_{\mathrm{H}} 7.50(\mathrm{~s}, 1 \mathrm{H}), 7.41-7.29(\mathrm{~m}, 4 \mathrm{H}), 7.25-7.18(\mathrm{~m}$, 2H), 5.27 (s, 2H) ppm; ${ }^{13} \mathbf{C}$ NMR (125 MHz, $\left.\mathrm{CDCl}_{3}, 298 \mathrm{~K}\right): \delta_{\mathrm{C}} 140.2,135.8,129.5,129.1$, 128.5, 128.0, 93.6, 56.8 ppm; $\mathbf{R}_{\mathbf{f}}\left(9: 1\right.$ hexanes/EtOAc; $\left.\mathrm{UV} / \mathrm{KMnO}_{4}\right): 0.41$.

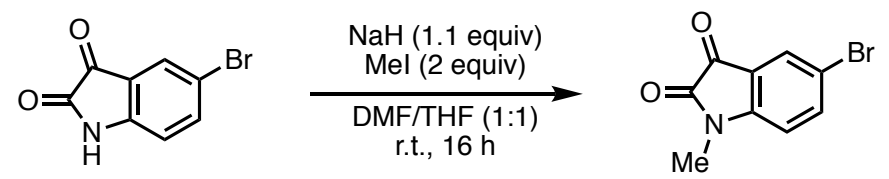

5-Bromo-1-methylindoline-2,3-dione (S20) (CAS: 2058-72-2): To a 50-mL flask were added 4-bromoisatin (1.4 g, $6.0 \mathrm{mmol}, 1.0$ equiv), THF (12 mL), and DMF (12 mL) (total reaction volume $=24 \mathrm{~mL}$ of a 1:1 DMF/THF mixture, $0.25 \mathrm{M})$. Sodium hydride $(0.26 \mathrm{~g}$ of a $60 \% \mathrm{w} / \mathrm{w}$ dispersion in mineral oil, $6.6 \mathrm{mmol}, 1.1$ equiv) was added and the reaction was stirred at r.t. for $15 \mathrm{~min}$. Iodomethane $(0.75 \mathrm{~mL}, 12 \mathrm{mmol}, 2.0$ equiv) was added and the reaction was stirred at r.t. for $16 \mathrm{~h}$. The reaction was quenched with sat. aq. $\mathrm{NH}_{4} \mathrm{Cl}$ and extracted with EtOAc $(\times 3)$. The organic fractions were combined, washed with brine $(\times 1)$, dried over $\mathrm{MgSO}_{4}$, and concentrated. The crude residue was purified by flash column chromatography (gradient of 20-80\% EtOAc/hexanes) to yield S20 as an orange-red solid (1.0 g, $4.2 \mathrm{mmol}, 70 \%)$. Analytical data: ${ }^{52}$ ${ }^{1} \mathbf{H}$ NMR $\left(400 \mathrm{MHz}, \mathrm{CDCl}_{3}, 298 \mathrm{~K}\right): \delta_{\mathrm{H}} 7.77-7.63(\mathrm{~m}, 2 \mathrm{H}), 6.86-6.76(\mathrm{~m}, 1 \mathrm{H}), 3.25(\mathrm{~s}, 3 \mathrm{H})$ ppm; $\mathbf{R}_{\mathbf{f}}(8: 2$ hexanes/EtOAc; UV): 0.13.



5-Bromo-2-(piperidin-1-yl)pyridine (S21) (CAS: 24255-95-6): ${ }^{53}$ To a $25-\mathrm{mL}$ flask with a stir bar were added 2,5-dibromopyridine ( $1.4 \mathrm{~g}, 6.0 \mathrm{mmol}, 1.0$ equiv), copper(I) iodide $(0.11 \mathrm{~g}, 0.60$ mmol, $10 \mathrm{~mol} \%$ ), L-proline $(0.14 \mathrm{~g}, 1.2 \mathrm{mmol}, 20 \mathrm{~mol} \%)$, potassium carbonate $(1.7 \mathrm{~g}, 12$ mmol, 2.0 equiv), DMSO (3.5 mL, $1.7 \mathrm{M})$, and piperidine $(0.89 \mathrm{~mL}, 9.0 \mathrm{~mL}, 1.5$ equiv). The reaction was stirred at $80{ }^{\circ} \mathrm{C}$ for $40 \mathrm{~h}$. The reaction was cooled to r.t., quenched with $\mathrm{H}_{2} \mathrm{O}$, and extracted with EtOAc $(\times 3)$. The organic fractions were combined, washed with brine $(\times 1)$, dried over $\mathrm{MgSO}_{4}$, and concentrated. The crude residue was purified by flash column chromatography (gradient of 0-10\% EtOAc/hexanes) to yield S21 as a colourless oil (0.41 g, $1.7 \mathrm{mmol}, 28 \%$ ). Analytical data: ${ }^{54} \mathbf{H}$ NMR $\left(400 \mathrm{MHz}, \mathrm{CDCl}_{3}, 298 \mathrm{~K}\right): \delta_{\mathrm{H}} 8.16(\mathrm{~d}, J=2.5 \mathrm{~Hz}, 1 \mathrm{H}), 7.47(\mathrm{dd}, J=$ 9.0, 2.6 Hz, 1H), $6.53(\mathrm{~d}, J=9.2 \mathrm{~Hz}, 1 \mathrm{H}), 3.55-3.39(\mathrm{~m}, 4 \mathrm{H}), 1.72-1.51(\mathrm{~m}, 6 \mathrm{H}) \mathrm{ppm} ;{ }^{13} \mathbf{C} \mathbf{N M R}$ $\left(100 \mathrm{MHz}, \mathrm{CDCl}_{3}, 298 \mathrm{~K}\right): \delta_{\mathrm{C}} 158.1,148.4,139.5,108.4,106.6,46.4,25.4,24.6 \mathrm{ppm} ; \mathbf{R}_{\mathbf{f}}(9: 1$ hexanes/EtOAc; $\left.\mathrm{UV} / \mathrm{KMnO}_{4}\right): 0.57$.

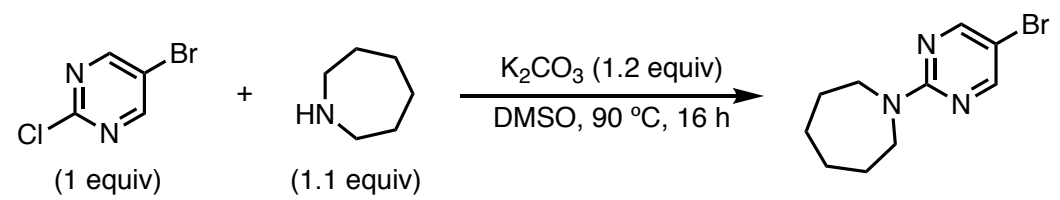

1-(5-Bromopyrimidin-2-yl)azepane (S22): To a 25-mL flask were added 5-bromo-2chloropyrimidine $(0.97 \mathrm{~g}, 5.0 \mathrm{mmol}, 1.0$ equiv), potassium carbonate $(0.83 \mathrm{~g}, 6.0 \mathrm{mmol}, 1.2$ equiv), DMSO $(10 \mathrm{~mL}, 0.50 \mathrm{M})$, and azepane $(0.62 \mathrm{~mL}, 5.5 \mathrm{mmol}, 1.1$ equiv). The reaction was stirred at $90{ }^{\circ} \mathrm{C}$ for $16 \mathrm{~h}$. The reaction was quenched with $\mathrm{H}_{2} \mathrm{O}$ and extracted with EtOAc $(\times 3)$, and the organic fractions were combined, washed with brine $(\times 1)$, dried over $\mathrm{MgSO}_{4}$, and 
concentrated. The crude residue was purified by flash column chromatography (gradient of 0 $30 \% \mathrm{EtOAc} /$ hexanes) to yield $\mathbf{S 2 2}$ as an off-white solid (1.2 g, $4.7 \mathrm{mmol}, 94 \%)$. Analytical data: ${ }^{55}{ }^{1} \mathbf{H}$ NMR $\left(400 \mathrm{MHz}, \mathrm{CDCl}_{3}, 298 \mathrm{~K}\right): \delta_{\mathrm{H}} 8.26(\mathrm{~s}, 2 \mathrm{H}), 3.73-3.66(\mathrm{~m}, 4 \mathrm{H}), 1.82-1.71(\mathrm{~m}$, 4H), 1.61-1.49 (m, 4H) ppm; ${ }^{13} \mathbf{C}$ NMR (100 MHz, $\left.\mathrm{CDCl}_{3}, 298 \mathrm{~K}\right): \delta_{\mathrm{C}} 159.9,157.9,104.9,47.5$, 27.8, 27.3 ppm; $\mathbf{R}_{\mathbf{f}}(8: 2$ hexanes/EtOAc; UV): 0.74.

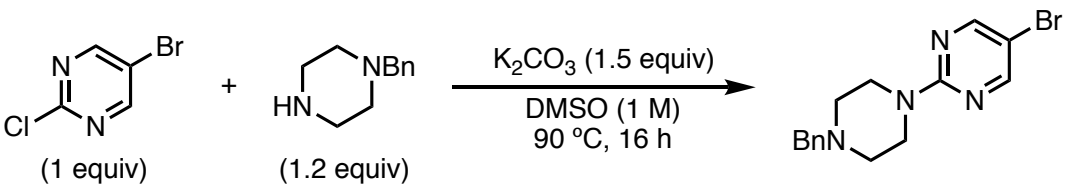

2-(4-Benzylpiperazin-1-yl)-5-bromopyrimidine (S23) (CAS: 1015241-96-9): To a 25-mL flask with a stir bar were added 5-bromo-2-chloropyrimidine ( $1.2 \mathrm{~g}, 6.0 \mathrm{mmol}, 1.0$ equiv), potassium carbonate $(1.2 \mathrm{~g}, 9.0 \mathrm{mmol}, 1.5$ equiv), DMSO $(6.0 \mathrm{~mL}, 1.0 \mathrm{M})$, and 1benzylpiperazine $\left(1.2 \mathrm{~mL}, 7.2 \mathrm{mmol}, 1.2\right.$ equiv), and the reaction was stirred at $90{ }^{\circ} \mathrm{C}$ for $16 \mathrm{~h}$. The reaction was cooled to r.t., quenched with $\mathrm{H}_{2} \mathrm{O}$, extracted with EtOAc $(\times 3)$. The organic fractions were combined, washed with brine $(\times 1)$, dried over $\mathrm{MgSO}_{4}$, and concentrated. The crude residue was purified by flash column chromatography (gradient of 20-60\%

EtOAc/hexanes) to yield $\mathbf{S 2 3}$ as an off-white solid (1.5 g, $4.5 \mathrm{mmol}, 75 \%)$. Analytical data: ${ }^{1} \mathbf{H}$ NMR $\left(400 \mathrm{MHz}, \mathrm{CDCl}_{3}, 298 \mathrm{~K}\right): \delta_{\mathrm{H}} 8.27(\mathrm{~s}, 2 \mathrm{H}), 7.38-7.24(\mathrm{~m}, 5 \mathrm{H}), 3.86-3.74(\mathrm{~m}, 4 \mathrm{H}), 3.55$ (s, 2H), 2.55-2.44 (m, 4H) ppm; ${ }^{13} \mathbf{C}$ NMR (100 MHz, $\left.\mathrm{CDCl}_{3}, 298 \mathrm{~K}\right): \delta_{\mathrm{C}} 160.0,158.0,138.0$, 129.3, 128.5, 127.4, 105.7, 63.2, 53.0, $44.1 \mathrm{ppm}$; HRMS $\mathrm{m} / z$ (DART): calcd for $\mathrm{C}_{15} \mathrm{H}_{18} \mathrm{BrN}_{4}$ $(\mathrm{M}+\mathrm{H}): 333.0715$; found 333.0708; IR (neat): 3029, 2998, 2954, 2943, 2906, 2874, 2862, 2820, 2802, 2777, 1576, 1523, 1492, 1451, 1391, 1322, 1303, 1148, 1123, 1005, 940, 738, $697 \mathrm{~cm}^{-1}$; m.p.: $81-82^{\circ} \mathrm{C} ; \mathbf{R}_{\mathbf{f}}(9: 1$ hexanes/EtOAc; UV): 0.15 .


1-Bromo-4-isopropoxybenzene (S24) (CAS: 6967-88-0): To a 50-mL flask with a stir bar were added 4-bromophenol ( $3.5 \mathrm{~g}, 20 \mathrm{mmol}, 1.0$ equiv), potassium carbonate $(4.1 \mathrm{~g}, 30 \mathrm{mmol}, 1.5$ equiv), DMSO (10 mL, 1.0 M), and 2-iodopropane (2.4 mL, $24 \mathrm{mmol}, 1.2$ equiv). The reaction was stirred at $60^{\circ} \mathrm{C}$ for $16 \mathrm{~h}$. The reaction was quenched with $\mathrm{H}_{2} \mathrm{O}$ and extracted with EtOAc $(\times 3)$. The organic fractions were combined, washed with brine $(\times 1)$, dried over $\mathrm{MgSO}_{4}$, and concentrated. The crude residue was purified by flash column chromatography (gradient of 0 5\% EtOAc/hexanes) to yield $\mathbf{S 2 4}$ as a colourless oil (3.5 g, $16 \mathrm{mmol}, 80 \%)$. Analytical data: ${ }^{56}{ }^{1} \mathbf{H}$ NMR (400 MHz, $\left.\mathrm{CDCl}_{3}, 298 \mathrm{~K}\right): \delta_{\mathrm{H}} 7.44-7.30(\mathrm{~m}, 2 \mathrm{H}), 6.85-6.70(\mathrm{~m}, 2 \mathrm{H}), 4.50$ (septet, $J=6.0$ $\mathrm{Hz}, 1 \mathrm{H}), 1.32(\mathrm{~d}, J=6.1 \mathrm{~Hz}, 6 \mathrm{H}) \mathrm{ppm} ;{ }^{13} \mathbf{C} \mathbf{N M R}\left(100 \mathrm{MHz}, \mathrm{CDCl}_{3}, 298 \mathrm{~K}\right): \delta_{\mathrm{C}} 157.0,132.3$, 117.7, 112.5, 70.3, 21.9 ppm; $\mathbf{R}_{\mathbf{f}}\left(9: 1\right.$ hexanes/EtOAc; $\left.\mathrm{UV} / \mathrm{KMnO}_{4}\right): 0.76$. 


\section{F.3. Synthesis of starting materials for biologically relevant benzonitriles}
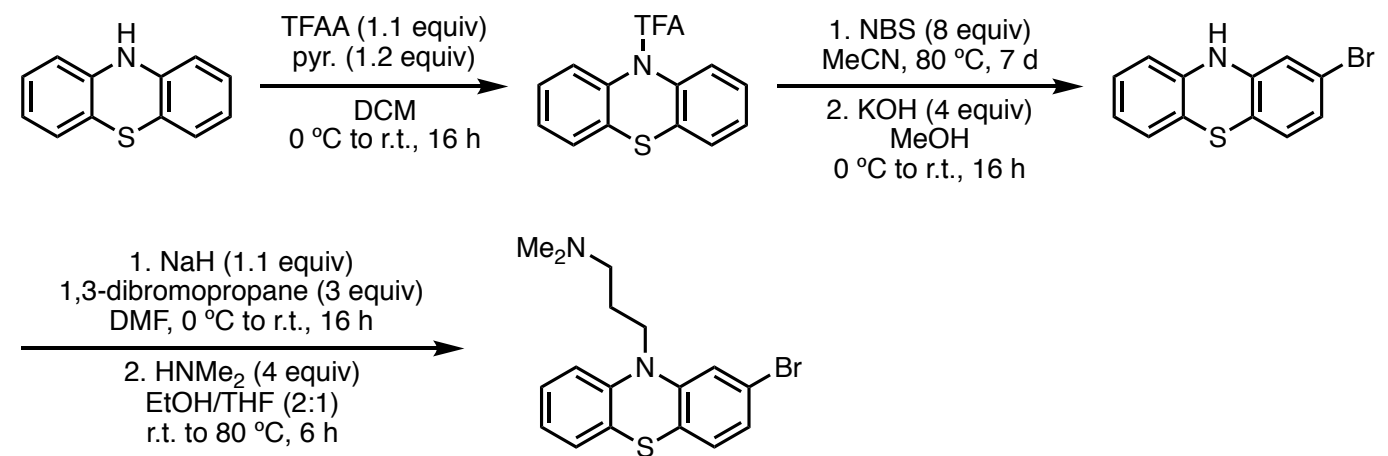

2,2,2-Trifluoro-1-(10H-phenothiazin-10-yl)ethan-1-one (S25) (CAS: 96124-86-6): To a 100mL flask were added phenothiazine $\left(6.0 \mathrm{~g}, 30 \mathrm{mmol}, 1.0\right.$ equiv) and $\mathrm{CH}_{2} \mathrm{Cl}_{2}(30 \mathrm{~mL}, 1.0 \mathrm{M})$. The solution was cooled to $0{ }^{\circ} \mathrm{C}$ and TFAA $(4.6 \mathrm{~mL}, 33 \mathrm{mmol}, 1.1$ equiv) and pyridine $(2.9 \mathrm{~mL}, 36$ mmol, 1.2 equiv) were added. The reaction was stirred at r.t. for $16 \mathrm{~h}$. The reaction was quenched with sat. aq. $\mathrm{NH}_{4} \mathrm{Cl}$ and extracted with $\mathrm{CH}_{2} \mathrm{Cl}_{2}(\times 3)$. The organic fractions were combined, washed with brine $(\times 1)$, dried over $\mathrm{MgSO}_{4}$, and concentrated to yield $\mathbf{S 2 5}$ as an offwhite solid (8.4 g, $28 \mathrm{mmol}, 93 \%)$. Analytical data: ${ }^{1} \mathbf{H}$ NMR $\left(400 \mathrm{MHz}, \mathrm{CDCl}_{3}, 298 \mathrm{~K}\right)$ : $\delta_{\mathrm{H}}$ 7.65-7.55 (m, 2H), 7.55-7.49 (m, 2H), 7.42-7.34 (m, 2H), 7.34-7.27 (m, 2H) ppm; ${ }^{19} \mathbf{F} \mathbf{N M R}$ $\left(376 \mathrm{MHz}, \mathrm{CDCl}_{3}, 298 \mathrm{~K}\right): \delta_{\mathrm{F}}-66.5 \mathrm{ppm}$; HRMS $\mathrm{m} / z$ (DART): calcd for $\mathrm{C}_{14} \mathrm{H}_{9} \mathrm{~F}_{3} \mathrm{NOS}(\mathrm{M}+\mathrm{H})$ : 296.0357; found 296.0356; $\mathbf{R}_{\mathbf{f}}(9: 1$ hexanes/EtOAc; UV): 0.50.

2-Bromo-10H-phenothiazine (S26) (CAS: 66820-95-9): Step 1: To a 100-mL flask with a stir bar were added S25 (3.5 g, $12 \mathrm{mmol}, 1.0$ equiv), MeCN (30 mL, $0.40 \mathrm{M}$ ), and $N$ bromosuccinimide (17 g, $96 \mathrm{mmol}, 8.0$ equiv) and the reaction was stirred at $80{ }^{\circ} \mathrm{C}$ for $7 \mathrm{~d}$. The reaction was cooled to r.t., quenched with $\mathrm{H}_{2} \mathrm{O}$, and extracted with EtOAc $(\times 3)$. The organic fractions were combined, washed with brine $(\times 1)$, dried over $\mathrm{MgSO}_{4}$, and concentrated. The crude residue was purified by flash column chromatography (gradient of 0-10\% EtOAc/hexanes) and the fractions containing product were collected and concentrated. This material was used in the next step without further purification.

Step 2: The material from step 1 was transferred to a 250-mL flask and $\mathrm{MeOH}(40 \mathrm{~mL}, 0.30 \mathrm{M})$ was added. The solution was cooled to $0{ }^{\circ} \mathrm{C}$ and $\mathrm{KOH}(1.9 \mathrm{~g}, 48 \mathrm{mmol}, 4.0$ equiv) was added. The reaction was stirred at r.t. for $16 \mathrm{~h}$. The reaction was concentrated and the concentrate was treated with sat. aq. $\mathrm{NH}_{4} \mathrm{Cl}(50 \mathrm{~mL})$. The solution was extracted with EtOAc $(\times 3)$ and the organic fractions were washed with brine $(\times 1)$, dried over $\mathrm{MgSO}_{4}$, and concentrated. The crude residue was purified by flash column chromatography (gradient of $0-20 \%$ EtOAc/hexanes) to yield S26 as an off-white solid (1.1 g, $4.0 \mathrm{mmol}, 33 \%$ over two steps). Analytical data: $:{ }^{57} \mathbf{H}$ NMR $\left(400 \mathrm{MHz}, \mathrm{DMSO}-d_{6}, 298 \mathrm{~K}\right): \delta_{\mathrm{H}} 8.76(\mathrm{~s}, 1 \mathrm{H}), 7.08-6.73(\mathrm{~m}, 6 \mathrm{H}), 6.71-6.62(\mathrm{~m}, 1 \mathrm{H})$ ppm; HRMS $m / z$ (DART): calcd for $\mathrm{C}_{12} \mathrm{H}_{9} \mathrm{BrNS}(\mathrm{M}+\mathrm{H}): 277.9639$; found 277.9634; $\mathbf{R}_{\mathbf{f}}(9: 1$ hexanes/EtOAc; UV): 0.46.

3-(2-Bromo-10H-phenothiazin-10-yl)-N,N-dimethylpropan-1-amine (bromopromazine) (S27) (CAS: 1757-73-9): Step 1: To a 50-mL flask with stir bar were added S26 (0.70 g, 2.5 mmol, 1.0 equiv) and DMF (7.5 mL, $0.33 \mathrm{M})$. The solution was cooled to $0{ }^{\circ} \mathrm{C}$ and sodium hydride $(0.11 \mathrm{~g}$ of a $60 \% \mathrm{w} / \mathrm{w}$ dispersion in mineral oil, $2.8 \mathrm{mmol}, 1.1$ equiv) was added. The 
reaction was stirred at $0{ }^{\circ} \mathrm{C}$ for $15 \mathrm{~min}$, then 1,3-dibromopropane was added $(0.76 \mathrm{~mL}, 7.5 \mathrm{mmol}$, 3.0 equiv). The reaction was stirred at r.t. for $16 \mathrm{~h}$. The reaction was quenched with sat. aq. $\mathrm{NH}_{4} \mathrm{Cl}$ and extracted with EtOAc $(\times 3)$, and the organic fractions were combined, washed with brine $(\times 1)$, dried over $\mathrm{MgSO}_{4}$, and concentrated. The crude residue was purified by flash column chromatography (gradient of $0-10 \%$ EtOAc/hexanes) and the fractions containing product were combined and concentrated to yield crude material which was used in the next step without further purification.

Step 2: The crude residue from step 1 was transferred to a $25-\mathrm{mL}$ flask and EtOH $(10 \mathrm{~mL})$ and THF $(5.0 \mathrm{~mL})$ were added. Dimethylamine $\left(1.3 \mathrm{~mL}\right.$ of a $40 \% \mathrm{w} / \mathrm{w}$ solution in $\mathrm{H}_{2} \mathrm{O}, 10 \mathrm{mmol}, 4.0$ equiv) was added and the reaction was stirred at $80{ }^{\circ} \mathrm{C}$ for $6 \mathrm{~h}$. The reaction was cooled to r.t. and quenched with $\mathrm{H}_{2} \mathrm{O}$. The solution was extracted with EtOAc $(\times 3)$ and the organic fractions were combined, washed with brine $(\times 1)$, dried over $\mathrm{MgSO}_{4}$, and concentrated. The crude residue was purified by flash column chromatography (gradient of 50-100\% EtOAc/hexanes) to yield S27 as a colourless oil $\left(0.13 \mathrm{~g}, 0.36 \mathrm{mmol}, 14 \%\right.$ over two steps). Analytical data: ${ }^{36}{ }^{1} \mathbf{H}$ NMR (400 MHz, $\left.\mathrm{CDCl}_{3}, 298 \mathrm{~K}\right): \delta_{\mathrm{H}} 7.20-7.08(\mathrm{~m}, 2 \mathrm{H}), 7.06-6.99(\mathrm{~m}, 2 \mathrm{H}), 6.99-6.84(\mathrm{~m}, 3 \mathrm{H}), 3.88(\mathrm{dd}, J=7.0$, $7.0 \mathrm{~Hz}, 2 \mathrm{H}), 2.39$ (dd, $J=7.0,7.0 \mathrm{~Hz}, 2 \mathrm{H}), 2.21$ (s, 6H), 1.93 (pent, $J=7.0 \mathrm{~Hz}, 2 \mathrm{H}) \mathrm{ppm} ;{ }^{13} \mathbf{C}$ NMR $\left(100 \mathrm{MHz}, \mathrm{CDCl}_{3}, 298 \mathrm{~K}\right): \delta_{\mathrm{C}} 148.8,144.6,128.2,127.5,127.4,125.2,124.9,124.3$, 122.9, 121.1, 118.7, 115.9, 57.0, 45.6, 45.5, $25.1 \mathrm{ppm}$; HRMS $m / z$ (DART): calcd for $\mathrm{C}_{17} \mathrm{H}_{20} \mathrm{Br}_{1} \mathrm{~N}_{2} \mathrm{~S}(\mathrm{M}+\mathrm{H}): 363.0531$; found 363.0529; $\mathbf{R}_{\mathbf{f}}(50 \%$ EtOAc/hexanes; UV): 0.05.

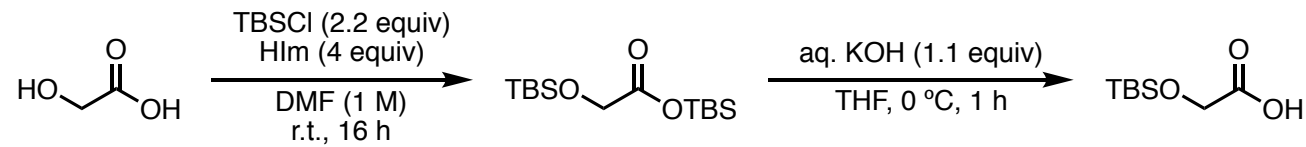

tert-Butyldimethylsilyl 2-((tert-butyldimethylsilyl)oxy)acetate (S28) (CAS: 67226-76-0): To a flame-dried 50-mL flask with a stir bar were added glycolic acid (0.91 g, $12 \mathrm{mmol}, 1.0$ equiv), TBSCl (3.9 g, $26 \mathrm{mmol}, 2.2$ equiv), imidazole ( $3.3 \mathrm{~g}, 48 \mathrm{mmol}, 4.0$ equiv), and DMF (12 mL, 1.0 $M)$. The reaction was stirred at r.t. for $16 \mathrm{~h}$. The reaction was poured into $\mathrm{H}_{2} \mathrm{O}(120 \mathrm{~mL})$ and the solution was extracted with hexanes $(\times 3)$. The organic fractions were combined, washed with sat. aq. $\mathrm{NaHCO}_{3}(\times 1)$ and brine $(\times 1)$, dried over $\mathrm{MgSO}_{4}$, and concentrated to yield $\mathbf{S 2 8}$ as a white solid (3.0 g, $9.8 \mathrm{mmol}, 82 \%)$. Analytical data: ${ }^{58} \mathbf{H}$ NMR (500 MHz, CDCl, $\left.298 \mathrm{~K}\right): \delta_{\mathrm{H}} 4.19$ (s, 2H), 0.93 (s, 9H), 0.92 (s, 9H), 0.28 (s, 6H), 0.10 (s, 6H) ppm; ${ }^{13} \mathbf{C}$ NMR (125 MHz, CDCl 3,298 $\mathrm{K}): \delta_{\mathrm{C}} 172.1,62.5,25.9,25.7,18.6,17.8,-4.6,-5.3 \mathrm{ppm}$.

2-((tert-Butyldimethylsilyl)oxy)acetic acid (S29) (CAS: 105459-05-0): To a 100-mL flask was added S28 (1.8 g, $6.0 \mathrm{mmol}, 1.0$ equiv) and THF (30 mL, $0.20 \mathrm{M})$. The solution was cooled to 0 ${ }^{\circ} \mathrm{C}$ and $2.0 \mathrm{M}$ aq. $\mathrm{KOH}(3.3 \mathrm{~mL}, 6.6 \mathrm{mmol})$ was added dropwise. The reaction was stirred at $0{ }^{\circ} \mathrm{C}$ for $1 \mathrm{~h}$. The reaction was poured into $\mathrm{H}_{2} \mathrm{O}(200 \mathrm{~mL})$ and extracted with $\mathrm{Et}_{2} \mathrm{O}(\times 3)$, and the organic layers were discarded. The aqueous layer was acidified with $1.0 \mathrm{M} \mathrm{HCl}$ to bring the solution to $\mathrm{pH} 2-3$. The solution was extracted with $\mathrm{Et}_{2} \mathrm{O}(\times 2)$ and the organic layers were combined, washed with $\mathrm{H}_{2} \mathrm{O}(\times 1)$ and brine $(\times 1)$, dried over $\mathrm{MgSO}_{4}$, and concentrated to yield S29 as colourless crystals $(0.51 \mathrm{~g}, 2.7 \mathrm{mmol}, 45 \%)$. Analytical data: ${ }^{59}{ }^{1} \mathbf{H}$ NMR (400 MHz, $\left.\mathrm{CDCl}_{3}, 298 \mathrm{~K}\right): \delta_{\mathrm{H}} 4.22(\mathrm{~s}, 2 \mathrm{H}), 0.91(\mathrm{~s}, 9 \mathrm{H}), 0.09$ (s, 6H) ppm; ${ }^{13} \mathbf{C}$ NMR $\left(100 \mathrm{MHz}, \mathrm{CDCl}_{3}\right.$, $298 \mathrm{~K}): \delta_{\mathrm{C}} 172.1,62.5,25.9,18.6,-5.3 \mathrm{ppm}$. 


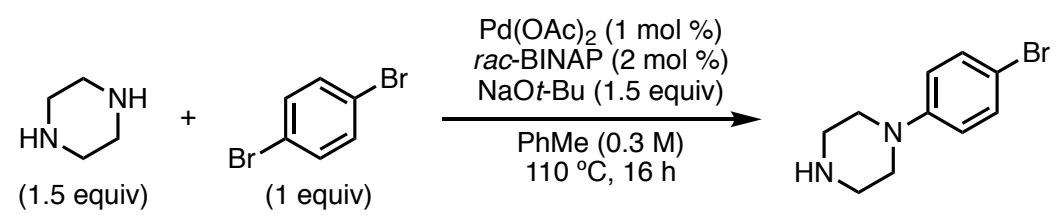

1-(4-Bromophenyl)piperazine (S30) (CAS: 66698-28-0): To a 100-mL flask were added piperazine (1.3 g, $15 \mathrm{mmol}, 1.5$ equiv), 1,4-dibromobenzene ( $2.4 \mathrm{~g}, 10 \mathrm{mmol}, 1.0$ equiv), palladium(II) acetate (22 mg, $0.10 \mathrm{mmol}, 1.0 \mathrm{~mol} \%)$, rac-BINAP (0.12 g, $0.20 \mathrm{mmol}, 2.0 \mathrm{~mol}$ $\%$ ), sodium tert-butoxide (1.4 g, $15 \mathrm{mmol}, 1.5$ equiv), and $\mathrm{PhMe}(30 \mathrm{~mL}, 0.30 \mathrm{M})$. The reaction was stirred at $110^{\circ} \mathrm{C}$ for $16 \mathrm{~h}$. The reaction was cooled to r.t., quenched with sat. aq. $\mathrm{NH}_{4} \mathrm{Cl}$, and extracted with EtOAc $(\times 3)$. The organic fractions were combined, washed with brine $(\times 1)$, dried over $\mathrm{MgSO}_{4}$, and concentrated. The crude residue was purified by flash column chromatography (gradient of $100 / 0 / 0$ to $85 / 14 / 1 \mathrm{CH}_{2} \mathrm{Cl}_{2} / \mathrm{MeOH} / \mathrm{NEt}_{3}$ ) to yield $\mathbf{S 3 0}$ as a white solid (0.90 g, 3.7 mmol, 37\%). Analytical data: ${ }^{60}{ }^{1} \mathbf{H}$ NMR (400 MHz, $\left.\mathrm{CDCl}_{3}, 298 \mathrm{~K}\right): \delta_{\mathrm{H}} 7.39-7.28(\mathrm{~m}, 2 \mathrm{H})$, 6.84-6.73 (m, 2H), 3.16-2.96 (m, 8H), 1.68 (br s, 1H) ppm; ${ }^{13} \mathbf{C} \mathbf{N M R}\left(100 \mathrm{MHz}, \mathrm{CDCl}_{3}, 298\right.$ $\mathrm{K}): \delta_{\mathrm{C}} 151.1,132.0,117.8,111.9,50.4,46.2 \mathrm{ppm} ; \mathbf{R}_{\mathbf{f}}\left(10 \% \mathrm{MeOH} / \mathrm{CH}_{2} \mathrm{Cl}_{2} ; \mathrm{UV}\right): 0.07$.



1-(4-(4-Bromophenyl)piperazin-1-yl)-2-((tert-butyldimethylsilyl)oxy)ethan-1-one (S31): To a flame-dried $25-\mathrm{mL}$ flask with a stir bar were added 2-((tert-butyldimethylsilyl)oxy)acetic acid (0.29 g, $1.5 \mathrm{mmol}, 1.0$ equiv), 1-(4-bromophenyl)piperazine (0.36 g, $1.5 \mathrm{mmol}, 1.0$ equiv), $N$-(3dimethylaminopropyl)- $N^{\prime}$-ethylcarbodiimide hydrochloride $(0.29 \mathrm{~g}, 1.5 \mathrm{mmol}, 1.0$ equiv), and 4dimethylaminopyridine $(0.18 \mathrm{~g}, 1.5 \mathrm{mmol}, 1.0$ equiv). The flask was sealed and evacuated and backfilled with $\mathrm{N}_{2}(\times 3)$. The flask was placed in a $0{ }^{\circ} \mathrm{C}$ cooling bath and $\mathrm{CH}_{2} \mathrm{Cl}_{2}(7.5 \mathrm{~mL}$, $0.20 \mathrm{M}$ ) was added. The reaction was stirred at $0{ }^{\circ} \mathrm{C}$ for $1 \mathrm{~h}$, then at r.t. for $15 \mathrm{~h}$. The reaction was quenched with $\mathrm{H}_{2} \mathrm{O}$ and extracted with EtOAc $(\times 3)$. The organic fractions were combined, washed with brine $(\times 1)$, dried over $\mathrm{MgSO}_{4}$, and concentrated. The crude residue was purified by flash column chromatography (gradient of 20-50\% EtOAc/hexanes) to yield $\mathbf{S 3 1}$ as a white solid (0.34 g, $0.82 \mathrm{mmol}, 55 \%)$. Analytical data: ${ }^{1} \mathbf{H} \mathbf{~ N M R}\left(400 \mathrm{MHz}, \mathrm{CDCl}_{3}, 298 \mathrm{~K}\right): \delta_{\mathrm{H}} 7.43-7.29$ $(\mathrm{m}, 2 \mathrm{H}), 6.86-6.69(\mathrm{~m}, 2 \mathrm{H}), 4.32(\mathrm{~s}, 2 \mathrm{H}), 3.86-3.61(\mathrm{~m}, 4 \mathrm{H}), 3.24-3.04(\mathrm{~m}, 4 \mathrm{H}), 0.90(\mathrm{~s}, 9 \mathrm{H})$, $0.11(\mathrm{~s}, 6 \mathrm{H}) \mathrm{ppm} ;{ }^{13} \mathbf{C} \mathbf{N M R}\left(100 \mathrm{MHz}, \mathrm{CDCl}_{3}, 298 \mathrm{~K}\right): \delta_{\mathrm{C}} 169.4,150.2,132.2,118.3,112.8$, 64.3, 49.9, 49.3, 45.1, 41.8, 25.9, 18.4, -5.3 ppm; HRMS $m / z$ (DART): calcd for $\mathrm{C}_{18} \mathrm{H}_{30} \mathrm{BrN}_{2} \mathrm{OSi}(\mathrm{M}+\mathrm{H})$ : 413.1260; found: 413.1259; IR (neat): 2951, 2920, 2880, 2853, 1659, 1494, 1434, 1230, 1154, 1030, 834, 824, $773 \mathrm{~cm}^{-1}$; m.p.: $83-84{ }^{\circ} \mathrm{C} ; \mathbf{R}_{\mathbf{f}}(6: 4$ hexanes/EtOAc; UV): 0.54 . 
F.4. Synthesis of alternate aryl electrophiles

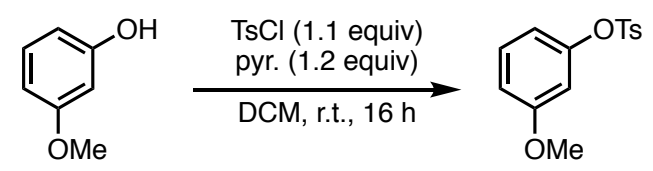

3-Methoxyphenyl 4-methylbenzenesulfonate (11b) (CAS: 3899-92-1): To a 50-mL flask with a stir bar were added tosyl chloride ( $4.2 \mathrm{~g}, 22 \mathrm{mmol}, 1.1$ equiv), $\mathrm{CH}_{2} \mathrm{Cl}_{2}(20 \mathrm{~mL}, 1.0 \mathrm{M}), 3-$ methoxyphenol (2.2 mL, $20 \mathrm{mmol}, 1.0$ equiv), and pyridine (1.9 mL, $24 \mathrm{mmol}, 1.2$ equiv). The reaction was stirred at r.t. for $16 \mathrm{~h}$. The reaction was quenched with $\mathrm{H}_{2} \mathrm{O}$ and extracted with $\mathrm{CH}_{2} \mathrm{Cl}_{2}(\times 3)$. The organic fractions were combined, washed with brine $(\times 1)$, dried over $\mathrm{MgSO}_{4}$, and concentrated. The crude residue was purified by flash column chromatography (gradient of 0-30\% EtOAc/hexanes) to yield the product as a colourless oil $(5.1 \mathrm{~g}, 18 \mathrm{mmol}, 90 \%)$.

Analytical data: ${ }^{61}{ }^{1} \mathbf{H}$ NMR (400 MHz, $\left.\mathrm{CDCl}_{3}, 298 \mathrm{~K}\right)$ : 7.75-7.69 (m, 2H), 7.34-7.28 (m, 2H), 7.20-7.12 (m, 1H), 6.81-6.75 (m, 1H), 6.58-6.52 (m, 2H), $3.72(\mathrm{~s}, 3 \mathrm{H}), 2.45(\mathrm{~s}, 3 \mathrm{H}) \mathrm{ppm} ;{ }^{13} \mathbf{C}$ NMR $\left(100 \mathrm{MHz}, \mathrm{CDCl}_{3}, 298 \mathrm{~K}\right): \delta_{\mathrm{C}} 160.6,150.7,145.4,132.7,130.0,129.9,128.7,114.5$, 113.2, 108.4, 55.6, 21.8 ppm; $\mathbf{R}_{\mathbf{f}}\left(9: 1\right.$ hexanes/EtOAc; $\left.\mathrm{UV} / \mathrm{KMnO}_{4}\right): 0.21$.



3-Methoxyphenyl trifluoromethanesulfonate (S32): A flame-dried 25-mL flask with stir bar was brought into a glovebox and $\mathrm{Tf}_{2} \mathrm{O}(1.6 \mathrm{~g}, 5.5 \mathrm{mmol}, 1.1$ equiv) was added. The flask was sealed and removed from the box and DCM $(5.0 \mathrm{~mL}, 1.0 \mathrm{M})$ was added, and the solution was cooled to $0{ }^{\circ} \mathrm{C}$. Pyridine $(0.49 \mathrm{~mL}, 6.0 \mathrm{mmol}, 1.2$ equiv) was added, followed by 3 -

methoxyphenol $(0.55 \mathrm{~mL}, 5.0 \mathrm{mmol}, 1.0$ equiv), and the reaction was stirred at r.t. for $16 \mathrm{~h}$. The reaction was quenched with $\mathrm{H}_{2} \mathrm{O}$ and extracted with DCM $(\times 3)$, and the organic fractions were combined, washed with brine $(\times 1)$, dried over $\mathrm{MgSO}_{4}$, and concentrated. The crude residue was purified by flash column chromatography (gradient of 0-5\% EtOAc/hexanes) to yield S32 as a slightly coloured oil (1.1 g, $4.3 \mathrm{mmol}, 86 \%)$. Analytical data: ${ }^{62}{ }^{1} \mathbf{H}$ NMR (400 MHz, $\mathrm{CDCl}_{3}, 298$ $\mathrm{K}): \delta_{\mathrm{H}} 7.34(\mathrm{t}, J=8.3 \mathrm{~Hz}, 1 \mathrm{H}), 6.95-6.90(\mathrm{~m}, 1 \mathrm{H}), 6.89-6.85(\mathrm{~m}, 1 \mathrm{H}), 6.81(\mathrm{t}, J=2.4 \mathrm{~Hz}, 1 \mathrm{H})$, 3.83 (s, 3H) ppm; $\left.{ }^{13} \mathbf{C ~ N M R ~ ( 1 0 0 ~ M H z , ~} \mathrm{CDCl}_{3}, 298 \mathrm{~K}\right): 160.9,150.3,130.5,123.5,118.7$ (q, $J=$ $318.7 \mathrm{ppm}), 114.1,113.2,107.5,55.7 \mathrm{ppm} ; \mathbf{R}_{\mathbf{f}}(9: 1$ hexanes/EtOAc; UV): 0.60.



5,6,7,8-Tetrahydronaphthalen-2-yl 4-methylbenzenesulfonate (S33): To a 50-mL flask with stir bar was added 5,6,7,8-tetrahydronaphthalen-2-ol (1.5 g, $10 \mathrm{mmol}, 1.0$ equiv), tosyl chloride (2.3 g, $12 \mathrm{mmol}, 1.2$ equiv), DCM (20 mL, $0.50 \mathrm{M}$ ), and pyridine (1.2 mL, $15 \mathrm{mmol}, 1.5 \mathrm{equiv})$, and the reaction was stirred at r.t. for $16 \mathrm{~h}$. The reaction was concentrated and purified by flash column chromatography (gradient of $0-20 \%$ EtOAc/hexanes) to yield $\mathbf{S 3 3}$ as an off-white solid (1.6 g, $6.0 \mathrm{mmol}, 60 \%)$. Analytical data: ${ }^{63}{ }^{1} \mathbf{H}$ NMR $\left(500 \mathrm{MHz}, \mathrm{CDCl}_{3}, 298 \mathrm{~K}\right): \delta_{\mathrm{H}} 7.77-7.67$ (m, 2H), 7.36-7.27 (m, 2H), 6.98-6.87 (m, 1H), 6.76-6.68 (m, 1H), 6.66-6.57 (m, 1H), 2.75$2.61(\mathrm{~m}, 4 \mathrm{H}), 2.45(\mathrm{~s}, 3 \mathrm{H}), 1.80-1.69(\mathrm{~m}, 4 \mathrm{H}) \mathrm{ppm} ;{ }^{13} \mathbf{C} \mathbf{N M R}\left(125 \mathrm{MHz}, \mathrm{CDCl}_{3}, 298 \mathrm{~K}\right): \delta_{\mathrm{C}}$ $147.3,145.2$, 138.9, 136.2, 132.9, 130.1, 129.8, 128.7, 122.7, 119.3, 29.5, 29.0, 23.0, 22.8, 21.9 ppm; $\mathbf{R}_{\mathbf{f}}(9: 1$ hexanes/EtOAc; UV): 0.50. 


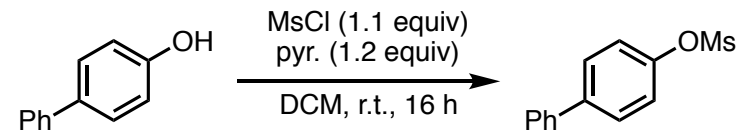

[1,1'-Biphenyl]-4-yl methanesulfonate (S34) (CAS: 129236-45-9): To a 25-mL flask was added 4-phenylphenol (1.4 g, $8.0 \mathrm{mmol}, 1.0$ equiv), DCM (8.0 mL, $1.0 \mathrm{M})$, mesyl chloride $(0.68$ $\mathrm{mL}, 8.8 \mathrm{mmol}, 1.1$ equiv), and pyridine $(0.78 \mathrm{~mL}, 9.6 \mathrm{mmol}, 1.2$ equiv). The reaction was stirred at r.t. for $16 \mathrm{~h}$, then was concentrated and purified by flash column chromatography (gradient of 10-60\% EtOAc/hexanes) to yield S34 as a tan solid (0.64 g, $2.6 \mathrm{mmol}, 33 \%)$. Analytical data: ${ }^{64}$ ${ }^{1} \mathbf{H}$ NMR $\left(500 \mathrm{MHz}, \mathrm{CDCl}_{3}, 298 \mathrm{~K}\right): \delta_{\mathrm{H}} 7.70-7.59(\mathrm{~m}, 2 \mathrm{H}), 7.59-7.51(\mathrm{~m}, 2 \mathrm{H}), 7.51-7.42(\mathrm{~m}$, 2H), 7.42-7.32 (m, 3H), $3.18(\mathrm{~s}, 3 \mathrm{H}) \mathrm{ppm} ;{ }^{13} \mathbf{C ~ N M R}\left(125 \mathrm{MHz}, \mathrm{CDCl}_{3}, 298 \mathrm{~K}\right): \delta_{\mathrm{C}} 148.7$, 140.8, 139.9, 129.1, 128.8, 127.9, 127.3, 122.4, 37.5 ppm; $\mathbf{R}_{\mathbf{f}}(9: 1$ hexanes/EtOAc; UV): 0.26.



(1 equiv)

(2 equiv)

2-(4-Chlorophenyl)-5,5-dimethyl-1,3-dioxane (S35) (CAS: 6283-10-9): To a 50-mL flask with a stir bar was added 4-chlorobenzaldehyde (1.1 g, $8.0 \mathrm{mmol}, 1.0$ equiv), 2,2-dimethylpropane1,3-diol (1.7 g, $16 \mathrm{mmol}, 2.0$ equiv), para-toluenesulfonic acid monohydrate ( $76 \mathrm{mg}, 0.40 \mathrm{mmol}$, 0.050 equiv), and PhMe (40 mL, $0.20 \mathrm{M})$. The flask was fitted with a Dean-Stark apparatus and the reaction was refluxed at $140^{\circ} \mathrm{C}$ for $16 \mathrm{~h}$. The reaction was cooled to r.t. and concentrated, and the concentrate was purified by flash column chromatography (gradient of $0-10 \%$ EtOAc/hexanes) to yield S35 as an off-white solid (1.4 g, $6.2 \mathrm{mmol}, 72 \%)$. Analytical data: ${ }^{65}{ }^{1} \mathbf{H}$ NMR $\left(500 \mathrm{MHz}, \mathrm{CDCl}_{3}, 298 \mathrm{~K}\right): \delta_{\mathrm{H}} 7.48-7.40(\mathrm{~m}, 2 \mathrm{H}), 7.38-7.31(\mathrm{~m}, 2 \mathrm{H}), 5.36(\mathrm{~s}, 1 \mathrm{H}), 3.80-$ $3.72(\mathrm{~m}, 2 \mathrm{H}), 3.68-3.59$ (m, 2H), 1.28 (s, 3H), 0.80 (s, 3H) ppm; ${ }^{13} \mathbf{C}$ NMR $\left(125 \mathrm{MHz}, \mathrm{CDCl}_{3}\right.$, $298 \mathrm{~K}): \delta_{\mathrm{C}} 137.2,134.8,128.6,127.8,101.1,77.8,30.4,23.2,22.0$ ppm; $\mathbf{R}_{\mathbf{f}}(9: 1$ hexanes/EtOAc; $\left.\mathrm{UV} / \mathrm{KMnO}_{4}\right): 0.62$. 


\section{G. Preparation and calibration of GC-MS standards}

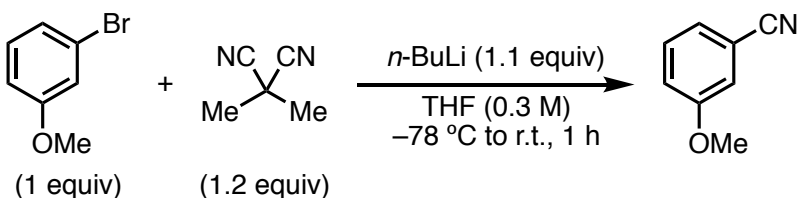

3-Methoxybenzonitrile (2a) (CAS: 1527-89-5): ${ }^{19}$ To a flame-dried $25-\mathrm{mL}$ flask with a stir bar were added 3-bromoanisole $(0.38 \mathrm{~mL}, 3.0 \mathrm{mmol}, 1.0$ equiv) and THF $(10 \mathrm{~mL}, 0.30 \mathrm{M})$. The solution was cooled to $-78{ }^{\circ} \mathrm{C}, n$-butyllithium $(2.1 \mathrm{~mL}$ of a $1.6 \mathrm{M}$ solution in hexanes, $3.3 \mathrm{mmol}$, 1.1 equiv) was added dropwise, and the reaction was stirred at $-78^{\circ} \mathrm{C}$ for $1 \mathrm{~h}$. The reaction was briefly opened to air and dimethylmalononitrile $(0.34 \mathrm{~g}, 3.6 \mathrm{mmol}, 1.2$ equiv $)$ was added. The reaction was stirred at r.t. for $1 \mathrm{~h}$. The reaction was opened to air, quenched with sat. aq. $\mathrm{NH}_{4} \mathrm{Cl}$, and extracted with EtOAc $(\times 3)$. The organic fractions were combined, washed with brine $(\times 1)$, dried over $\mathrm{MgSO}_{4}$, and concentrated. The crude residue was purified by flash column chromatography (gradient of 0-20\% EtOAc/hexanes) to yield 2a as a colourless oil ( $0.15 \mathrm{~g}, 1.1$ mmol, 37\%). Analytical data: ${ }^{6}{ }^{1} \mathbf{H}$ NMR (400 MHz, $\left.\mathrm{CDCl}_{3}, 298 \mathrm{~K}\right): \delta_{\mathrm{H}} 7.40-7.29(\mathrm{~m}, 1 \mathrm{H})$, 7.25-7.18 (m, 1H), 7.16-7.04 (m, 2H), 3.82 (s, 3H) ppm; ${ }^{13} \mathbf{C ~ N M R ~ ( 1 0 0 ~ M H z , ~ C D C l ~} 3$, $\left.298 \mathrm{~K}\right)$ : $\delta_{\mathrm{C}} 159.7,130.4,124.5,119.3,118.8,116.9,113.2,55.6$ ppm; $\mathbf{R}_{\mathbf{f}}(9: 1$ hexanes/EtOAc; UV): 0.39.

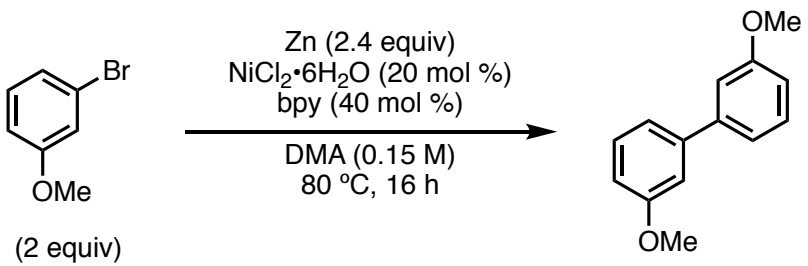

3,3'-Dimethoxy-1,1'-biphenyl (S36) (CAS: 6161-50-8): To a flame-dried 50-mL flask with a stir bar were added $\mathrm{Zn}(0)$ dust $\left(0.31 \mathrm{~g}, 4.8 \mathrm{mmol}, 2.4\right.$ equiv), $\mathrm{NiCl}_{2} \bullet 6 \mathrm{H}_{2} \mathrm{O}(95 \mathrm{mg}, 0.40 \mathrm{mmol}, 20$ mol \%), and 2,2'-bipyridine (0.12 g, $0.80 \mathrm{mmol}, 40 \mathrm{~mol} \%)$. The flask was evacuated and backfilled with $\mathrm{N}_{2}(\times 3)$ and DMA $(13 \mathrm{~mL}, 0.15 \mathrm{M})$ was added, followed by 3-bromoanisole $\left(0.51 \mathrm{~mL}, 4.0 \mathrm{mmol}, 2.0\right.$ equiv). The reaction was stirred at $80^{\circ} \mathrm{C}$ for $16 \mathrm{~h}$. The reaction was cooled to r.t., quenched with $\mathrm{H}_{2} \mathrm{O}$, and extracted with EtOAc $(\times 3)$. The organic fractions were combined, washed with brine $(\times 1)$, dried over $\mathrm{MgSO}_{4}$, and concentrated. The crude residue was purified by flash column chromatography (gradient of 0-10\% EtOAc/hexanes) to yield $\mathbf{S 3 6}$ as a colourless oil $(0.17 \mathrm{~g}, 0.79 \mathrm{mmol}, 40 \%)$. Analytical data: ${ }^{67}{ }^{1} \mathbf{H} \mathbf{N M R}\left(400 \mathrm{MHz}, \mathrm{CDCl}_{3}, 298 \mathrm{~K}\right)$ : $\delta_{\mathrm{H}} 7.41-7.31(\mathrm{~m}, 2 \mathrm{H}), 7.24-7.09(\mathrm{~m}, 4 \mathrm{H}), 6.96-6.86(\mathrm{~m}, 2 \mathrm{H}), 3.88(\mathrm{~s}, 6 \mathrm{H}) \mathrm{ppm} ;{ }^{13} \mathbf{C}$ NMR $(100$ $\left.\mathrm{MHz}, \mathrm{CDCl}_{3}, 298 \mathrm{~K}\right): \delta_{\mathrm{C}} 159.9,142.7,129.8,119.7,113.0,112.9,55.3 \mathrm{ppm} ; \mathbf{R}_{\mathbf{f}}(5 \%$ EtOAc/hexanes; UV): 0.48 .

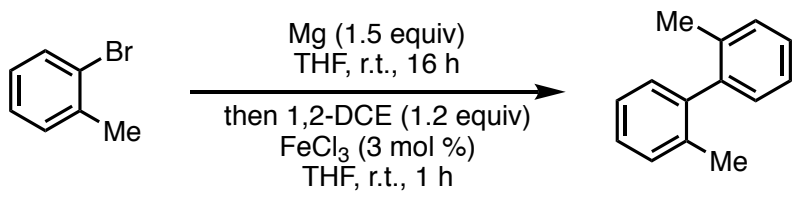

2,2'-Dimethyl-1,1'-biphenyl (S37) (CAS: 605-39-0): ${ }^{68}$ To a 50-mL flask with a stir bar were added magnesium( 0$)$ turnings $(0.36 \mathrm{~g}, 15 \mathrm{mmol}, 1.5$ equiv) and the flask was flame-dried and cooled under vacuum. The flask was backfilled with $\mathrm{N}_{2}$ and THF $(20 \mathrm{~mL}, 0.50 \mathrm{M})$ was added. The magnesium(0) turnings were activated with 1,2-dibromoethane (ca. $0.020 \mathrm{~mL}, 0.23 \mathrm{mmol}$, 0.023 equiv), then 2-bromotoluene ( $1.2 \mathrm{~mL}, 10 \mathrm{mmol}, 1.0$ equiv) was added portionwise over 30 
min at r.t. After addition, the reaction was stirred at r.t. for an additional $1 \mathrm{~h}$. To a separate flamedried 100-mL flask with a stir bar were added iron(III) chloride (49 mg, $0.30 \mathrm{mmol}, 0.030$ equiv), THF (20 mL), and 1,2-dichloroethane ( $0.95 \mathrm{~mL}, 12 \mathrm{mmol}, 1.2$ equiv), followed by the solution of 2-tolylmagnesium bromide in THF, and the reaction was stirred at r.t. for $1 \mathrm{~h}$. The reaction was quenched with sat. aq. $\mathrm{NH}_{4} \mathrm{Cl}$ and extracted with EtOAc $(\times 3)$. The organic fractions were combined, washed with brine $(\times 1)$, dried over $\mathrm{MgSO}_{4}$, and concentrated. The crude residue was purified by flash column chromatography (gradient of 0-10\% EtOAc/hexanes) to yield S37 as a colourless oil $(0.31 \mathrm{~g}, 1.7 \mathrm{mmol}, 17 \%)$. Analytical data: ${ }^{69}{ }^{1} \mathbf{H} \mathbf{~ N M R}\left(400 \mathrm{MHz}, \mathrm{CDCl}_{3}\right.$, $298 \mathrm{~K}): \delta_{\mathrm{H}} 7.51-7.24(\mathrm{~m}, 6 \mathrm{H}), 7.24-7.06(\mathrm{~m}, 2 \mathrm{H}), 2.13(\mathrm{~s}, 6 \mathrm{H}) \mathrm{ppm} ;{ }^{13} \mathbf{C}$ NMR $(100 \mathrm{MHz}$, $\left.\mathrm{CDCl}_{3}, 298 \mathrm{~K}\right): \delta_{\mathrm{C}} 141.7,135.9,129.9,129.3,127.2,125.6,19.9$ ppm; $\mathbf{R}_{\mathbf{f}}(9: 1$ hexanes/EtOAc; $\left.\mathrm{UV} / \mathrm{KMnO}_{4}\right): 0.87$. 


\section{H. X-ray data for complex 4}

Table 1. Crystal data and structure refinement for d19133_a_5.

Identification code

Empirical formula

Formula weight

Temperature

Wavelength

Crystal system

Space group

Unit cell dimensions

Volume

Z

Density (calculated)

Absorption coefficient

$\mathrm{F}(000)$

Crystal size

Theta range for data collection

Index ranges

Reflections collected

Independent reflections

Completeness to theta $=67.679^{\circ}$

Absorption correction

Max. and min. transmission

Refinement method

Data / restraints / parameters

Goodness-of-fit on $\mathrm{F}^{2}$

Final $\mathrm{R}$ indices [I $>2 \operatorname{sigma}(\mathrm{I})]$

$\mathrm{R}$ indices (all data)

Extinction coefficient

Largest diff. peak and hole d19133_a_5

C17 H15 Br N2 Ni

385.93

150(2) K

$1.54178 \AA$

Triclinic

P-1

$\mathrm{a}=9.1066(4) \AA$

$\mathrm{a}=83.294(3)^{\circ}$.

$\mathrm{b}=10.0998(4) \AA$

$\mathrm{b}=77.888(3)^{\circ}$.

$\mathrm{c}=18.5656(6) \AA$

$\mathrm{g}=63.691(3)^{\circ}$.

1496.07(11) $\AA^{3}$

4

$1.713 \mathrm{Mg} / \mathrm{m}^{3}$

$4.931 \mathrm{~mm}^{-1}$

776

$0.150 \times 0.020 \times 0.020 \mathrm{~mm}^{3}$

2.435 to $68.342^{\circ}$.

$-10<=\mathrm{h}<=10,-11<=\mathrm{k}<=12,0<=\mathrm{l}<=22$

5377

$5377[\mathrm{R}(\mathrm{int})=0.1517]$

$98.9 \%$

Semi-empirical from equivalents

0.7529 and 0.5830

Full-matrix least-squares on $\mathrm{F}^{2}$

5377 / 0 / 382

1.034

$\mathrm{R} 1=0.0715, \mathrm{wR} 2=0.1537$

$\mathrm{R} 1=0.1058, \mathrm{wR} 2=0.1762$

$0.00077(17)$

1.137 and $-1.020 \mathrm{e} . \AA^{-3}$ 
Table 2. Atomic coordinates ( $\times 10^{4}$ ) and equivalent isotropic displacement parameters $\left(\AA^{2} \times 10^{3}\right)$ for d19133_a_5. U(eq) is defined as one third of the trace of the orthogonalized $\mathrm{U}^{\mathrm{ij}}$ tensor.

\begin{tabular}{|c|c|c|c|c|}
\hline & $\mathrm{x}$ & $\mathrm{y}$ & $\mathrm{z}$ & $\mathrm{U}(\mathrm{eq})$ \\
\hline $\mathrm{Ni}(1 \mathrm{~A})$ & $5512(2)$ & 2711(2) & $863(1)$ & $30(1)$ \\
\hline $\operatorname{Br}(1 \mathrm{~A})$ & $6520(2)$ & 2143(1) & 1950(1) & $43(1)$ \\
\hline $\mathrm{N}(1 \mathrm{~A})$ & $7616(11)$ & $2449(9)$ & 199(4) & $33(2)$ \\
\hline $\mathrm{N}(2 \mathrm{~A})$ & $4651(10)$ & $3292(9)$ & $-41(4)$ & $29(2)$ \\
\hline$C(1 \mathrm{~A})$ & $9122(14)$ & $2007(12)$ & $378(6)$ & $40(3)$ \\
\hline$C(2 A)$ & $10540(14)$ & $1634(12)$ & $-135(6)$ & $42(3)$ \\
\hline$C(3 A)$ & $10514(14)$ & $1660(13)$ & $-869(7)$ & $48(3)$ \\
\hline$C(4 A)$ & $8962(13)$ & $2136(12)$ & $-1073(6)$ & $41(3)$ \\
\hline$C(5 \mathrm{~A})$ & $7543(13)$ & $2546(11)$ & $-524(6)$ & $33(2)$ \\
\hline$C(6 A)$ & $5815(12)$ & $3112(10)$ & $-659(5)$ & $31(2)$ \\
\hline$C(7 A)$ & $5405(13)$ & $3484(11)$ & $-1367(5)$ & $35(2)$ \\
\hline $\mathrm{C}(8 \mathrm{~A})$ & $3760(13)$ & $4013(12)$ & $-1425(6)$ & $38(3)$ \\
\hline$C(9 A)$ & 2583(12) & 4190(12) & $-801(6)$ & $36(2)$ \\
\hline $\mathrm{C}(10 \mathrm{~A})$ & $3052(13)$ & $3813(12)$ & $-117(6)$ & $35(2)$ \\
\hline $\mathrm{C}(11 \mathrm{~A})$ & $3499(13)$ & $2812(11)$ & $1405(5)$ & $32(2)$ \\
\hline $\mathrm{C}(12 \mathrm{~A})$ & $3098(14)$ & $1602(11)$ & 1407(6) & $36(2)$ \\
\hline$C(13 \mathrm{~A})$ & $1475(14)$ & $1765(13)$ & $1725(6)$ & $43(3)$ \\
\hline$C(14 \mathrm{~A})$ & $268(14)$ & 3101(14) & 2032(6) & $46(3)$ \\
\hline$C(15 A)$ & $680(14)$ & $4274(12)$ & 2047(6) & $43(3)$ \\
\hline$C(16 \mathrm{~A})$ & $2285(13)$ & $4116(12)$ & $1742(6)$ & $40(3)$ \\
\hline $\mathrm{C}(17 \mathrm{~A})$ & 4363(15) & $167(12)$ & 1064(7) & $47(3)$ \\
\hline $\mathrm{Ni}(1 \mathrm{~B})$ & $1819(2)$ & $7634(2)$ & $4125(1)$ & $27(1)$ \\
\hline $\operatorname{Br}(1 \mathrm{~B})$ & $1357(1)$ & 6964(1) & $3090(1)$ & $40(1)$ \\
\hline $\mathrm{N}(1 \mathrm{~B})$ & $-68(9)$ & $7440(8)$ & $4816(4)$ & $26(2)$ \\
\hline $\mathrm{N}(2 \mathrm{~B})$ & 2116(9) & $8289(8)$ & 4993(4) & $28(2)$ \\
\hline $\mathrm{C}(1 \mathrm{~B})$ & $-1115(12)$ & $6949(11)$ & $4667(5)$ & $31(2)$ \\
\hline $\mathrm{C}(2 \mathrm{~B})$ & $-2245(13)$ & $6674(11)$ & $5223(6)$ & $37(2)$ \\
\hline $\mathrm{C}(3 \mathrm{~B})$ & $-2279(13)$ & $6889(12)$ & $5955(6)$ & $40(3)$ \\
\hline $\mathrm{C}(4 \mathrm{~B})$ & $-1241(12)$ & $7429(12)$ & 6086(6) & $36(2)$ \\
\hline $\mathrm{C}(5 \mathrm{~B})$ & $-141(13)$ & $7709(11)$ & $5527(5)$ & $33(2)$ \\
\hline$C(6 B)$ & 1001(11) & $8296(10)$ & $5610(5)$ & $27(2)$ \\
\hline
\end{tabular}




\begin{tabular}{lllll}
$\mathrm{C}(7 \mathrm{~B})$ & $1026(12)$ & $8808(11)$ & $6274(5)$ & $35(2)$ \\
$\mathrm{C}(8 \mathrm{~B})$ & $2184(12)$ & $9320(12)$ & $6309(6)$ & $35(2)$ \\
$\mathrm{C}(9 \mathrm{~B})$ & $3312(13)$ & $9299(11)$ & $5684(6)$ & $36(2)$ \\
$\mathrm{C}(10 \mathrm{~B})$ & $3214(12)$ & $8801(12)$ & $5033(5)$ & $31(2)$ \\
$\mathrm{C}(11 \mathrm{~B})$ & $3791(12)$ & $7669(11)$ & $3550(5)$ & $30(2)$ \\
$\mathrm{C}(12 \mathrm{~B})$ & $5322(13)$ & $6432(12)$ & $3592(6)$ & $36(2)$ \\
$\mathrm{C}(13 \mathrm{~B})$ & $6764(12)$ & $6573(12)$ & $3309(5)$ & $36(2)$ \\
$\mathrm{C}(14 \mathrm{~B})$ & $6789(13)$ & $7865(13)$ & $2966(6)$ & $40(3)$ \\
$\mathrm{C}(15 \mathrm{~B})$ & $5278(14)$ & $9042(13)$ & $2883(6)$ & $43(3)$ \\
$\mathrm{C}(16 \mathrm{~B})$ & $3816(13)$ & $8932(12)$ & $3175(6)$ & $38(3)$ \\
$\mathrm{C}(17 \mathrm{~B})$ & $5381(14)$ & $4985(12)$ & $3931(7)$ & $47(3)$ \\
& & & & \\
\hline
\end{tabular}


Table 3. Bond lengths $[\AA ̊]$ and angles $\left[{ }^{\circ}\right]$ for d19133_a_5.

\begin{tabular}{|c|c|}
\hline $\mathrm{Ni}(1 \mathrm{~A})-\mathrm{C}(11 \mathrm{~A})$ & $1.862(10)$ \\
\hline $\mathrm{Ni}(1 \mathrm{~A})-\mathrm{N}(2 \mathrm{~A})$ & $1.922(8)$ \\
\hline $\mathrm{Ni}(1 \mathrm{~A})-\mathrm{N}(1 \mathrm{~A})$ & 1.974(9) \\
\hline $\mathrm{Ni}(1 \mathrm{~A})-\mathrm{Br}(1 \mathrm{~A})$ & $2.2988(19)$ \\
\hline $\mathrm{N}(1 \mathrm{~A})-\mathrm{C}(1 \mathrm{~A})$ & $1.343(13)$ \\
\hline $\mathrm{N}(1 \mathrm{~A})-\mathrm{C}(5 \mathrm{~A})$ & $1.348(13)$ \\
\hline $\mathrm{N}(2 \mathrm{~A})-\mathrm{C}(10 \mathrm{~A})$ & $1.344(13)$ \\
\hline $\mathrm{N}(2 \mathrm{~A})-\mathrm{C}(6 \mathrm{~A})$ & $1.355(12)$ \\
\hline$C(1 \mathrm{~A})-\mathrm{C}(2 \mathrm{~A})$ & $1.361(15)$ \\
\hline $\mathrm{C}(1 \mathrm{~A})-\mathrm{H}(1 \mathrm{AA})$ & 0.9500 \\
\hline$C(2 A)-C(3 A)$ & $1.365(16)$ \\
\hline $\mathrm{C}(2 \mathrm{~A})-\mathrm{H}(2 \mathrm{AA})$ & 0.9500 \\
\hline$C(3 \mathrm{~A})-\mathrm{C}(4 \mathrm{~A})$ & $1.397(16)$ \\
\hline $\mathrm{C}(3 \mathrm{~A})-\mathrm{H}(3 \mathrm{AA})$ & 0.9500 \\
\hline$C(4 A)-C(5 A)$ & $1.397(14)$ \\
\hline $\mathrm{C}(4 \mathrm{~A})-\mathrm{H}(4 \mathrm{AA})$ & 0.9500 \\
\hline$C(5 A)-C(6 A)$ & $1.483(14)$ \\
\hline $\mathrm{C}(6 \mathrm{~A})-\mathrm{C}(7 \mathrm{~A})$ & $1.406(14)$ \\
\hline $\mathrm{C}(7 \mathrm{~A})-\mathrm{C}(8 \mathrm{~A})$ & $1.373(15)$ \\
\hline $\mathrm{C}(7 \mathrm{~A})-\mathrm{H}(7 \mathrm{AA})$ & 0.9500 \\
\hline $\mathrm{C}(8 \mathrm{~A})-\mathrm{C}(9 \mathrm{~A})$ & $1.370(15)$ \\
\hline $\mathrm{C}(8 \mathrm{~A})-\mathrm{H}(8 \mathrm{AA})$ & 0.9500 \\
\hline$C(9 A)-C(10 A)$ & $1.383(14)$ \\
\hline $\mathrm{C}(9 \mathrm{~A})-\mathrm{H}(9 \mathrm{AA})$ & 0.9500 \\
\hline $\mathrm{C}(10 \mathrm{~A})-\mathrm{H}(10 \mathrm{~A})$ & 0.9500 \\
\hline$C(11 A)-C(16 A)$ & $1.400(14)$ \\
\hline $\mathrm{C}(11 \mathrm{~A})-\mathrm{C}(12 \mathrm{~A})$ & $1.421(14)$ \\
\hline $\mathrm{C}(12 \mathrm{~A})-\mathrm{C}(13 \mathrm{~A})$ & $1.414(15)$ \\
\hline $\mathrm{C}(12 \mathrm{~A})-\mathrm{C}(17 \mathrm{~A})$ & $1.503(15)$ \\
\hline $\mathrm{C}(13 \mathrm{~A})-\mathrm{C}(14 \mathrm{~A})$ & $1.399(15)$ \\
\hline $\mathrm{C}(13 \mathrm{~A})-\mathrm{H}(13 \mathrm{~A})$ & 0.9500 \\
\hline $\mathrm{C}(14 \mathrm{~A})-\mathrm{C}(15 \mathrm{~A})$ & $1.397(16)$ \\
\hline $\mathrm{C}(14 \mathrm{~A})-\mathrm{H}(14 \mathrm{~A})$ & 0.9500 \\
\hline$C(15 A)-C(16 A)$ & $1.396(15)$ \\
\hline
\end{tabular}




\begin{tabular}{|c|c|}
\hline $\mathrm{C}(15 \mathrm{~A})-\mathrm{H}(15 \mathrm{~A})$ & 0.9500 \\
\hline$C(16 A)-H(16 A)$ & 0.9500 \\
\hline $\mathrm{C}(17 \mathrm{~A})-\mathrm{H}(17 \mathrm{~A})$ & 0.9800 \\
\hline $\mathrm{C}(17 \mathrm{~A})-\mathrm{H}(17 \mathrm{~B})$ & 0.9800 \\
\hline $\mathrm{C}(17 \mathrm{~A})-\mathrm{H}(17 \mathrm{C})$ & 0.9800 \\
\hline $\mathrm{Ni}(1 \mathrm{~B})-\mathrm{C}(11 \mathrm{~B})$ & $1.899(10)$ \\
\hline $\mathrm{Ni}(1 \mathrm{~B})-\mathrm{N}(2 \mathrm{~B})$ & $1.923(8)$ \\
\hline $\mathrm{Ni}(1 \mathrm{~B})-\mathrm{N}(1 \mathrm{~B})$ & $1.988(8)$ \\
\hline $\mathrm{Ni}(1 \mathrm{~B})-\mathrm{Br}(1 \mathrm{~B})$ & $2.2854(18)$ \\
\hline $\mathrm{N}(1 \mathrm{~B})-\mathrm{C}(1 \mathrm{~B})$ & $1.338(12)$ \\
\hline $\mathrm{N}(1 \mathrm{~B})-\mathrm{C}(5 \mathrm{~B})$ & $1.361(11)$ \\
\hline $\mathrm{N}(2 \mathrm{~B})-\mathrm{C}(10 \mathrm{~B})$ & $1.330(12)$ \\
\hline $\mathrm{N}(2 \mathrm{~B})-\mathrm{C}(6 \mathrm{~B})$ & $1.360(12)$ \\
\hline $\mathrm{C}(1 \mathrm{~B})-\mathrm{C}(2 \mathrm{~B})$ & $1.393(14)$ \\
\hline $\mathrm{C}(1 \mathrm{~B})-\mathrm{H}(1 \mathrm{BA})$ & 0.9500 \\
\hline$C(2 B)-C(3 B)$ & $1.394(15)$ \\
\hline $\mathrm{C}(2 \mathrm{~B})-\mathrm{H}(2 \mathrm{BA})$ & 0.9500 \\
\hline $\mathrm{C}(3 \mathrm{~B})-\mathrm{C}(4 \mathrm{~B})$ & $1.354(15)$ \\
\hline $\mathrm{C}(3 \mathrm{~B})-\mathrm{H}(3 \mathrm{BA})$ & 0.9500 \\
\hline $\mathrm{C}(4 \mathrm{~B})-\mathrm{C}(5 \mathrm{~B})$ & $1.381(15)$ \\
\hline $\mathrm{C}(4 \mathrm{~B})-\mathrm{H}(4 \mathrm{BA})$ & 0.9500 \\
\hline $\mathrm{C}(5 \mathrm{~B})-\mathrm{C}(6 \mathrm{~B})$ & $1.447(14)$ \\
\hline $\mathrm{C}(6 \mathrm{~B})-\mathrm{C}(7 \mathrm{~B})$ & $1.398(13)$ \\
\hline $\mathrm{C}(7 \mathrm{~B})-\mathrm{C}(8 \mathrm{~B})$ & $1.381(15)$ \\
\hline $\mathrm{C}(7 \mathrm{~B})-\mathrm{H}(7 \mathrm{BA})$ & 0.9500 \\
\hline $\mathrm{C}(8 \mathrm{~B})-\mathrm{C}(9 \mathrm{~B})$ & $1.374(15)$ \\
\hline $\mathrm{C}(8 \mathrm{~B})-\mathrm{H}(8 \mathrm{BA})$ & 0.9500 \\
\hline $\mathrm{C}(9 \mathrm{~B})-\mathrm{C}(10 \mathrm{~B})$ & $1.396(13)$ \\
\hline $\mathrm{C}(9 \mathrm{~B})-\mathrm{H}(9 \mathrm{BA})$ & 0.9500 \\
\hline $\mathrm{C}(10 \mathrm{~B})-\mathrm{H}(10 \mathrm{~B})$ & 0.9500 \\
\hline$C(11 B)-C(16 B)$ & $1.388(15)$ \\
\hline $\mathrm{C}(11 \mathrm{~B})-\mathrm{C}(12 \mathrm{~B})$ & $1.410(14)$ \\
\hline$C(12 B)-C(13 B)$ & $1.370(15)$ \\
\hline$C(12 B)-C(17 B)$ & $1.505(16)$ \\
\hline$C(13 B)-C(14 B)$ & $1.392(15)$ \\
\hline $\mathrm{C}(13 \mathrm{~B})-\mathrm{H}(13 \mathrm{~B})$ & 0.9500 \\
\hline
\end{tabular}




\begin{tabular}{|c|c|}
\hline $\mathrm{C}(14 \mathrm{~B})-\mathrm{C}(15 \mathrm{~B})$ & $1.387(15)$ \\
\hline $\mathrm{C}(14 \mathrm{~B})-\mathrm{H}(14 \mathrm{~B})$ & 0.9500 \\
\hline$C(15 B)-C(16 B)$ & $1.376(15)$ \\
\hline $\mathrm{C}(15 \mathrm{~B})-\mathrm{H}(15 \mathrm{~B})$ & 0.9500 \\
\hline $\mathrm{C}(16 \mathrm{~B})-\mathrm{H}(16 \mathrm{~B})$ & 0.9500 \\
\hline $\mathrm{C}(17 \mathrm{~B})-\mathrm{H}(17 \mathrm{D})$ & 0.9800 \\
\hline $\mathrm{C}(17 \mathrm{~B})-\mathrm{H}(17 \mathrm{E})$ & 0.9800 \\
\hline $\mathrm{C}(17 \mathrm{~B})-\mathrm{H}(17 \mathrm{~F})$ & 0.9800 \\
\hline $\mathrm{C}(11 \mathrm{~A})-\mathrm{Ni}(1 \mathrm{~A})-\mathrm{N}(2 \mathrm{~A})$ & $92.8(4)$ \\
\hline $\mathrm{C}(11 \mathrm{~A})-\mathrm{Ni}(1 \mathrm{~A})-\mathrm{N}(1 \mathrm{~A})$ & $172.6(4)$ \\
\hline $\mathrm{N}(2 \mathrm{~A})-\mathrm{Ni}(1 \mathrm{~A})-\mathrm{N}(1 \mathrm{~A})$ & $82.4(3)$ \\
\hline $\mathrm{C}(11 \mathrm{~A})-\mathrm{Ni}(1 \mathrm{~A})-\mathrm{Br}(1 \mathrm{~A})$ & $87.7(3)$ \\
\hline $\mathrm{N}(2 \mathrm{~A})-\mathrm{Ni}(1 \mathrm{~A})-\mathrm{Br}(1 \mathrm{~A})$ & $177.0(2)$ \\
\hline $\mathrm{N}(1 \mathrm{~A})-\mathrm{Ni}(1 \mathrm{~A})-\mathrm{Br}(1 \mathrm{~A})$ & $97.3(2)$ \\
\hline$C(1 A)-N(1 A)-C(5 A)$ & $117.1(9)$ \\
\hline $\mathrm{C}(1 \mathrm{~A})-\mathrm{N}(1 \mathrm{~A})-\mathrm{Ni}(1 \mathrm{~A})$ & $127.9(7)$ \\
\hline $\mathrm{C}(5 \mathrm{~A})-\mathrm{N}(1 \mathrm{~A})-\mathrm{Ni}(1 \mathrm{~A})$ & $114.4(7)$ \\
\hline$C(10 A)-N(2 A)-C(6 A)$ & $118.3(9)$ \\
\hline $\mathrm{C}(10 \mathrm{~A})-\mathrm{N}(2 \mathrm{~A})-\mathrm{Ni}(1 \mathrm{~A})$ & $126.8(7)$ \\
\hline $\mathrm{C}(6 \mathrm{~A})-\mathrm{N}(2 \mathrm{~A})-\mathrm{Ni}(1 \mathrm{~A})$ & $115.0(7)$ \\
\hline $\mathrm{N}(1 \mathrm{~A})-\mathrm{C}(1 \mathrm{~A})-\mathrm{C}(2 \mathrm{~A})$ & $122.8(11)$ \\
\hline $\mathrm{N}(1 \mathrm{~A})-\mathrm{C}(1 \mathrm{~A})-\mathrm{H}(1 \mathrm{AA})$ & 118.6 \\
\hline $\mathrm{C}(2 \mathrm{~A})-\mathrm{C}(1 \mathrm{~A})-\mathrm{H}(1 \mathrm{AA})$ & 118.6 \\
\hline$C(1 A)-C(2 A)-C(3 A)$ & $121.4(10)$ \\
\hline $\mathrm{C}(1 \mathrm{~A})-\mathrm{C}(2 \mathrm{~A})-\mathrm{H}(2 \mathrm{AA})$ & 119.3 \\
\hline $\mathrm{C}(3 \mathrm{~A})-\mathrm{C}(2 \mathrm{~A})-\mathrm{H}(2 \mathrm{AA})$ & 119.3 \\
\hline$C(2 A)-C(3 A)-C(4 A)$ & $117.1(11)$ \\
\hline $\mathrm{C}(2 \mathrm{~A})-\mathrm{C}(3 \mathrm{~A})-\mathrm{H}(3 \mathrm{AA})$ & 121.4 \\
\hline $\mathrm{C}(4 \mathrm{~A})-\mathrm{C}(3 \mathrm{~A})-\mathrm{H}(3 \mathrm{AA})$ & 121.4 \\
\hline$C(3 A)-C(4 A)-C(5 A)$ & $118.9(11)$ \\
\hline $\mathrm{C}(3 \mathrm{~A})-\mathrm{C}(4 \mathrm{~A})-\mathrm{H}(4 \mathrm{AA})$ & 120.5 \\
\hline $\mathrm{C}(5 \mathrm{~A})-\mathrm{C}(4 \mathrm{~A})-\mathrm{H}(4 \mathrm{AA})$ & 120.5 \\
\hline $\mathrm{N}(1 \mathrm{~A})-\mathrm{C}(5 \mathrm{~A})-\mathrm{C}(4 \mathrm{~A})$ & $122.5(10)$ \\
\hline$N(1 A)-C(5 A)-C(6 A)$ & $112.5(9)$ \\
\hline$C(4 A)-C(5 A)-C(6 A)$ & $124.9(10)$ \\
\hline
\end{tabular}




\begin{tabular}{|c|c|}
\hline $\mathrm{N}(2 \mathrm{~A})-\mathrm{C}(6 \mathrm{~A})-\mathrm{C}(7 \mathrm{~A})$ & $122.5(9)$ \\
\hline $\mathrm{N}(2 \mathrm{~A})-\mathrm{C}(6 \mathrm{~A})-\mathrm{C}(5 \mathrm{~A})$ & $114.6(9)$ \\
\hline$C(7 A)-C(6 A)-C(5 A)$ & $122.8(9)$ \\
\hline $\mathrm{C}(8 \mathrm{~A})-\mathrm{C}(7 \mathrm{~A})-\mathrm{C}(6 \mathrm{~A})$ & $117.7(10)$ \\
\hline $\mathrm{C}(8 \mathrm{~A})-\mathrm{C}(7 \mathrm{~A})-\mathrm{H}(7 \mathrm{AA})$ & 121.2 \\
\hline$C(6 A)-C(7 A)-H(7 A A)$ & 121.2 \\
\hline $\mathrm{C}(9 \mathrm{~A})-\mathrm{C}(8 \mathrm{~A})-\mathrm{C}(7 \mathrm{~A})$ & $119.9(10)$ \\
\hline $\mathrm{C}(9 \mathrm{~A})-\mathrm{C}(8 \mathrm{~A})-\mathrm{H}(8 \mathrm{AA})$ & 120.1 \\
\hline $\mathrm{C}(7 \mathrm{~A})-\mathrm{C}(8 \mathrm{~A})-\mathrm{H}(8 \mathrm{AA})$ & 120.1 \\
\hline$C(8 A)-C(9 A)-C(10 A)$ & $120.1(10)$ \\
\hline $\mathrm{C}(8 \mathrm{~A})-\mathrm{C}(9 \mathrm{~A})-\mathrm{H}(9 \mathrm{AA})$ & 119.9 \\
\hline$C(10 A)-C(9 A)-H(9 A A)$ & 119.9 \\
\hline $\mathrm{N}(2 \mathrm{~A})-\mathrm{C}(10 \mathrm{~A})-\mathrm{C}(9 \mathrm{~A})$ & $121.5(10)$ \\
\hline $\mathrm{N}(2 \mathrm{~A})-\mathrm{C}(10 \mathrm{~A})-\mathrm{H}(10 \mathrm{~A})$ & 119.2 \\
\hline $\mathrm{C}(9 \mathrm{~A})-\mathrm{C}(10 \mathrm{~A})-\mathrm{H}(10 \mathrm{~A})$ & 119.2 \\
\hline$C(16 A)-C(11 A)-C(12 A)$ & $118.7(10)$ \\
\hline$C(16 A)-C(11 A)-N i(1 A)$ & $121.4(8)$ \\
\hline $\mathrm{C}(12 \mathrm{~A})-\mathrm{C}(11 \mathrm{~A})-\mathrm{Ni}(1 \mathrm{~A})$ & $119.5(8)$ \\
\hline$C(13 A)-C(12 A)-C(11 A)$ & $119.3(10)$ \\
\hline$C(13 A)-C(12 A)-C(17 A)$ & $119.8(10)$ \\
\hline$C(11 A)-C(12 A)-C(17 A)$ & $120.9(10)$ \\
\hline$C(14 A)-C(13 A)-C(12 A)$ & $120.7(11)$ \\
\hline $\mathrm{C}(14 \mathrm{~A})-\mathrm{C}(13 \mathrm{~A})-\mathrm{H}(13 \mathrm{~A})$ & 119.6 \\
\hline$C(12 A)-C(13 A)-H(13 A)$ & 119.6 \\
\hline$C(15 A)-C(14 A)-C(13 A)$ & $119.8(10)$ \\
\hline $\mathrm{C}(15 \mathrm{~A})-\mathrm{C}(14 \mathrm{~A})-\mathrm{H}(14 \mathrm{~A})$ & 120.1 \\
\hline $\mathrm{C}(13 \mathrm{~A})-\mathrm{C}(14 \mathrm{~A})-\mathrm{H}(14 \mathrm{~A})$ & 120.1 \\
\hline$C(16 A)-C(15 A)-C(14 A)$ & $119.8(10)$ \\
\hline$C(16 A)-C(15 A)-H(15 A)$ & 120.1 \\
\hline $\mathrm{C}(14 \mathrm{~A})-\mathrm{C}(15 \mathrm{~A})-\mathrm{H}(15 \mathrm{~A})$ & 120.1 \\
\hline$C(15 A)-C(16 A)-C(11 A)$ & $121.6(11)$ \\
\hline$C(15 A)-C(16 A)-H(16 A)$ & 119.2 \\
\hline$C(11 A)-C(16 A)-H(16 A)$ & 119.2 \\
\hline $\mathrm{C}(12 \mathrm{~A})-\mathrm{C}(17 \mathrm{~A})-\mathrm{H}(17 \mathrm{~A})$ & 109.5 \\
\hline $\mathrm{C}(12 \mathrm{~A})-\mathrm{C}(17 \mathrm{~A})-\mathrm{H}(17 \mathrm{~B})$ & 109.5 \\
\hline $\mathrm{H}(17 \mathrm{~A})-\mathrm{C}(17 \mathrm{~A})-\mathrm{H}(17 \mathrm{~B})$ & 109.5 \\
\hline
\end{tabular}




\begin{tabular}{|c|c|}
\hline $\mathrm{C}(12 \mathrm{~A})-\mathrm{C}(17 \mathrm{~A})-\mathrm{H}(17 \mathrm{C})$ & 109.5 \\
\hline $\mathrm{H}(17 \mathrm{~A})-\mathrm{C}(17 \mathrm{~A})-\mathrm{H}(17 \mathrm{C})$ & 109.5 \\
\hline $\mathrm{H}(17 \mathrm{~B})-\mathrm{C}(17 \mathrm{~A})-\mathrm{H}(17 \mathrm{C})$ & 109.5 \\
\hline $\mathrm{C}(11 \mathrm{~B})-\mathrm{Ni}(1 \mathrm{~B})-\mathrm{N}(2 \mathrm{~B})$ & $93.3(4)$ \\
\hline$C(11 B)-N i(1 B)-N(1 B)$ & $172.9(4)$ \\
\hline $\mathrm{N}(2 \mathrm{~B})-\mathrm{Ni}(1 \mathrm{~B})-\mathrm{N}(1 \mathrm{~B})$ & $82.5(3)$ \\
\hline $\mathrm{C}(11 \mathrm{~B})-\mathrm{Ni}(1 \mathrm{~B})-\mathrm{Br}(1 \mathrm{~B})$ & $87.7(3)$ \\
\hline $\mathrm{N}(2 \mathrm{~B})-\mathrm{Ni}(1 \mathrm{~B})-\mathrm{Br}(1 \mathrm{~B})$ & $177.2(2)$ \\
\hline $\mathrm{N}(1 \mathrm{~B})-\mathrm{Ni}(1 \mathrm{~B})-\mathrm{Br}(1 \mathrm{~B})$ & $96.7(2)$ \\
\hline$C(1 B)-N(1 B)-C(5 B)$ & $118.9(8)$ \\
\hline$C(1 B)-N(1 B)-N i(1 B)$ & $127.4(6)$ \\
\hline $\mathrm{C}(5 \mathrm{~B})-\mathrm{N}(1 \mathrm{~B})-\mathrm{Ni}(1 \mathrm{~B})$ & $113.3(7)$ \\
\hline $\mathrm{C}(10 \mathrm{~B})-\mathrm{N}(2 \mathrm{~B})-\mathrm{C}(6 \mathrm{~B})$ & $118.5(8)$ \\
\hline $\mathrm{C}(10 \mathrm{~B})-\mathrm{N}(2 \mathrm{~B})-\mathrm{Ni}(1 \mathrm{~B})$ & $126.8(7)$ \\
\hline $\mathrm{C}(6 \mathrm{~B})-\mathrm{N}(2 \mathrm{~B})-\mathrm{Ni}(1 \mathrm{~B})$ & $114.6(6)$ \\
\hline $\mathrm{N}(1 \mathrm{~B})-\mathrm{C}(1 \mathrm{~B})-\mathrm{C}(2 \mathrm{~B})$ & $121.7(9)$ \\
\hline $\mathrm{N}(1 \mathrm{~B})-\mathrm{C}(1 \mathrm{~B})-\mathrm{H}(1 \mathrm{BA})$ & 119.1 \\
\hline$C(2 B)-C(1 B)-H(1 B A)$ & 119.1 \\
\hline $\mathrm{C}(1 \mathrm{~B})-\mathrm{C}(2 \mathrm{~B})-\mathrm{C}(3 \mathrm{~B})$ & $119.6(10)$ \\
\hline $\mathrm{C}(1 \mathrm{~B})-\mathrm{C}(2 \mathrm{~B})-\mathrm{H}(2 \mathrm{BA})$ & 120.2 \\
\hline $\mathrm{C}(3 \mathrm{~B})-\mathrm{C}(2 \mathrm{~B})-\mathrm{H}(2 \mathrm{BA})$ & 120.2 \\
\hline $\mathrm{C}(4 \mathrm{~B})-\mathrm{C}(3 \mathrm{~B})-\mathrm{C}(2 \mathrm{~B})$ & $117.2(10)$ \\
\hline$C(4 B)-C(3 B)-H(3 B A)$ & 121.4 \\
\hline$C(2 B)-C(3 B)-H(3 B A)$ & 121.4 \\
\hline$C(3 B)-C(4 B)-C(5 B)$ & $122.2(10)$ \\
\hline$C(3 B)-C(4 B)-H(4 B A)$ & 118.9 \\
\hline $\mathrm{C}(5 \mathrm{~B})-\mathrm{C}(4 \mathrm{~B})-\mathrm{H}(4 \mathrm{BA})$ & 118.9 \\
\hline $\mathrm{N}(1 \mathrm{~B})-\mathrm{C}(5 \mathrm{~B})-\mathrm{C}(4 \mathrm{~B})$ & $120.2(9)$ \\
\hline $\mathrm{N}(1 \mathrm{~B})-\mathrm{C}(5 \mathrm{~B})-\mathrm{C}(6 \mathrm{~B})$ & $113.5(9)$ \\
\hline$C(4 B)-C(5 B)-C(6 B)$ & $126.3(9)$ \\
\hline$N(2 B)-C(6 B)-C(7 B)$ & $120.8(9)$ \\
\hline $\mathrm{N}(2 \mathrm{~B})-\mathrm{C}(6 \mathrm{~B})-\mathrm{C}(5 \mathrm{~B})$ & $115.4(8)$ \\
\hline$C(7 B)-C(6 B)-C(5 B)$ & $123.8(9)$ \\
\hline$C(8 B)-C(7 B)-C(6 B)$ & $120.1(10)$ \\
\hline $\mathrm{C}(8 \mathrm{~B})-\mathrm{C}(7 \mathrm{~B})-\mathrm{H}(7 \mathrm{BA})$ & 119.9 \\
\hline $\mathrm{C}(6 \mathrm{~B})-\mathrm{C}(7 \mathrm{~B})-\mathrm{H}(7 \mathrm{BA})$ & 119.9 \\
\hline
\end{tabular}




$\begin{array}{ll}\text { C(9B)-C(8B)-C(7B) } & 118.6(10) \\ \text { C(9B)-C(8B)-H(8BA) } & 120.7 \\ \text { C(7B)-C(8B)-H(8BA) } & 120.7 \\ \text { C(8B)-C(9B)-C(10B) } & 118.9(10) \\ \text { C(8B)-C(9B)-H(9BA) } & 120.6 \\ \text { C(10B)-C(9B)-H(9BA) } & 120.6 \\ \text { N(2B)-C(10B)-C(9B) } & 123.1(10) \\ \text { N(2B)-C(10B)-H(10B) } & 118.5 \\ \text { C(9B)-C(10B)-H(10B) } & 118.5 \\ \text { C(16B)-C(11B)-C(12B) } & 118.3(10) \\ \text { C(16B)-C(11B)-Ni(1B) } & 122.5(8) \\ \text { C(12B)-C(11B)-Ni(1B) } & 118.5(8) \\ \text { C(13B)-C(12B)-C(11B) } & 118.4(10) \\ \text { C(13B)-C(12B)-C(17B) } & 120.5(10) \\ \text { C(11B)-C(12B)-C(17B) } & 121.1(10) \\ \text { C(12B)-C(13B)-C(14B) } & 123.1(10) \\ \text { C(12B)-C(13B)-H(13B) } & 118.5 \\ \text { C(14B)-C(13B)-H(13B) } & 118.5 \\ \text { C(15B)-C(14B)-C(13B) } & 118.0(10) \\ \text { C(15B)-C(14B)-H(14B) } & 121.0 \\ \text { C(13B)-C(14B)-H(14B) } & 121.0 \\ \text { C(16B)-C(15B)-C(14B) } & 119.7(11) \\ \text { C(16B)-C(15B)-H(15B) } & 120.1 \\ \text { C(14B)-C(15B)-H(15B) } & 120.1 \\ \text { C(15B)-C(16B)-C(11B) } & 122.2(11) \\ \text { C(15B)-C(16B)-H(16B) } & 118.9 \\ \text { C(11B)-C(16B)-H(16B) } & 118.9 \\ \text { C(12B)-C(17B)-H(17D) } & 109.5 \\ \text { C(12B)-C(17B)-H(17E) } & 109.5 \\ \text { H(17D)-C(17B)-H(17E) } & 109.5 \\ \text { C(12B)-C(17B)-H(17F) } & 109.5 \\ \text { H(17D)-C(17B)-H(17F) } & 109.5 \\ \text { H(17E)-C(17B)-H(17F) } & 109.5 \\ & \\ & \end{array}$

Symmetry transformations used to generate equivalent atoms: 
Table 4. Anisotropic displacement parameters $\left(\AA^{2} \mathrm{x} 10^{3}\right)$ for d19133_a_5. The anisotropic displacement factor exponent takes the form: $-2 p^{2}\left[h^{2} a^{* 2} U^{11}+\ldots+2 h k a^{*} b^{*} U^{12}\right]$

\begin{tabular}{|c|c|c|c|c|c|c|}
\hline & $\mathrm{U}^{11}$ & $\mathrm{U}^{22}$ & $\mathrm{U}^{33}$ & $\mathrm{U}^{23}$ & $\mathrm{U}^{13}$ & $\mathrm{U}^{12}$ \\
\hline $\mathrm{Ni}(1 \mathrm{~A})$ & $31(1)$ & $34(1)$ & $27(1)$ & 2(1) & $-8(1)$ & $-16(1)$ \\
\hline $\operatorname{Br}(1 \mathrm{~A})$ & $48(1)$ & $57(1)$ & $35(1)$ & $9(1)$ & $-17(1)$ & $-32(1)$ \\
\hline $\mathrm{N}(1 \mathrm{~A})$ & $38(5)$ & $41(5)$ & $31(4)$ & $0(4)$ & $-18(4)$ & $-21(4)$ \\
\hline $\mathrm{N}(2 \mathrm{~A})$ & $29(5)$ & $34(4)$ & $29(4)$ & $-1(3)$ & $-1(4)$ & $-20(4)$ \\
\hline $\mathrm{C}(1 \mathrm{~A})$ & $41(7)$ & 41(6) & $42(6)$ & $6(5)$ & $-14(5)$ & $-20(5)$ \\
\hline $\mathrm{C}(2 \mathrm{~A})$ & $27(6)$ & $49(6)$ & $55(7)$ & $6(5)$ & $-12(5)$ & $-20(5)$ \\
\hline $\mathrm{C}(3 \mathrm{~A})$ & $25(6)$ & $60(8)$ & $54(7)$ & $-3(6)$ & $-3(5)$ & $-16(6)$ \\
\hline $\mathrm{C}(4 \mathrm{~A})$ & $34(6)$ & $46(7)$ & $43(6)$ & $-8(5)$ & $3(5)$ & $-20(5)$ \\
\hline$C(5 \mathrm{~A})$ & $34(6)$ & $34(5)$ & $35(5)$ & $-5(4)$ & $-10(4)$ & $-16(4)$ \\
\hline$C(6 A)$ & $34(6)$ & $29(5)$ & $36(5)$ & $-2(4)$ & $-6(4)$ & $-17(4)$ \\
\hline $\mathrm{C}(7 \mathrm{~A})$ & $43(7)$ & $38(6)$ & $29(5)$ & $4(4)$ & $-11(5)$ & $-22(5)$ \\
\hline $\mathrm{C}(8 \mathrm{~A})$ & $42(6)$ & $39(6)$ & $40(6)$ & $-6(5)$ & $-15(5)$ & $-18(5)$ \\
\hline $\mathrm{C}(9 \mathrm{~A})$ & $26(5)$ & $43(6)$ & $42(6)$ & $3(5)$ & $-16(5)$ & $-12(5)$ \\
\hline$C(10 A)$ & $34(6)$ & $42(6)$ & $35(6)$ & $5(5)$ & $-5(5)$ & $-25(5)$ \\
\hline $\mathrm{C}(11 \mathrm{~A})$ & $39(6)$ & $32(5)$ & $27(5)$ & $-4(4)$ & $-1(4)$ & $-18(5)$ \\
\hline $\mathrm{C}(12 \mathrm{~A})$ & $51(7)$ & $33(6)$ & $27(5)$ & $6(4)$ & $-18(5)$ & $-18(5)$ \\
\hline$C(13 \mathrm{~A})$ & $44(7)$ & $60(7)$ & $38(6)$ & $2(5)$ & $-21(5)$ & $-28(6)$ \\
\hline $\mathrm{C}(14 \mathrm{~A})$ & $30(6)$ & $72(8)$ & $45(6)$ & $6(6)$ & $-2(5)$ & $-35(6)$ \\
\hline$C(15 A)$ & $40(7)$ & $42(6)$ & $38(6)$ & $0(5)$ & $-9(5)$ & $-10(5)$ \\
\hline$C(16 A)$ & $34(6)$ & $40(6)$ & $42(6)$ & $-4(5)$ & $3(5)$ & $-16(5)$ \\
\hline $\mathrm{C}(17 \mathrm{~A})$ & $58(8)$ & $39(6)$ & $52(7)$ & $-7(5)$ & $-25(6)$ & $-19(6)$ \\
\hline Ni(1B) & $23(1)$ & $32(1)$ & $27(1)$ & $-3(1)$ & $-4(1)$ & $-13(1)$ \\
\hline $\operatorname{Br}(1 \mathrm{~B})$ & $34(1)$ & $61(1)$ & $32(1)$ & $-12(1)$ & $-1(1)$ & $-26(1)$ \\
\hline $\mathrm{N}(1 \mathrm{~B})$ & $23(4)$ & $32(4)$ & $22(4)$ & $-3(3)$ & $-3(3)$ & $-11(3)$ \\
\hline $\mathrm{N}(2 \mathrm{~B})$ & $19(4)$ & $32(4)$ & $32(4)$ & $-1(3)$ & $-3(3)$ & $-9(3)$ \\
\hline $\mathrm{C}(1 \mathrm{~B})$ & $30(5)$ & $43(6)$ & $26(5)$ & $-1(4)$ & $-6(4)$ & $-21(5)$ \\
\hline $\mathrm{C}(2 \mathrm{~B})$ & $31(6)$ & $30(5)$ & $52(6)$ & $6(5)$ & $-7(5)$ & $-17(5)$ \\
\hline$C(3 B)$ & $27(6)$ & $53(7)$ & $41(6)$ & $-2(5)$ & $4(5)$ & $-21(5)$ \\
\hline$C(4 B)$ & $24(5)$ & $50(7)$ & $29(5)$ & $3(5)$ & $-8(4)$ & $-10(5)$ \\
\hline $\mathrm{C}(5 \mathrm{~B})$ & $30(5)$ & $38(5)$ & $29(5)$ & $-7(4)$ & $-12(4)$ & $-9(5)$ \\
\hline $\mathrm{C}(6 \mathrm{~B})$ & $20(5)$ & $23(5)$ & $34(5)$ & $-1(4)$ & $-4(4)$ & $-5(4)$ \\
\hline
\end{tabular}




\begin{tabular}{lllllll}
$\mathrm{C}(7 \mathrm{~B})$ & $25(5)$ & $38(6)$ & $29(5)$ & $-2(4)$ & $-15(4)$ & $1(5)$ \\
$\mathrm{C}(8 \mathrm{~B})$ & $28(5)$ & $40(6)$ & $36(6)$ & $-10(5)$ & $-14(5)$ & $-6(5)$ \\
$\mathrm{C}(9 \mathrm{~B})$ & $28(6)$ & $45(6)$ & $40(6)$ & $-8(5)$ & $-10(5)$ & $-16(5)$ \\
$\mathrm{C}(10 \mathrm{~B})$ & $25(5)$ & $48(6)$ & $24(5)$ & $-7(4)$ & $-8(4)$ & $-15(5)$ \\
$\mathrm{C}(11 \mathrm{~B})$ & $23(5)$ & $33(5)$ & $29(5)$ & $1(4)$ & $-2(4)$ & $-10(4)$ \\
$\mathrm{C}(12 \mathrm{~B})$ & $32(6)$ & $34(6)$ & $36(6)$ & $-7(5)$ & $-11(5)$ & $-6(5)$ \\
$\mathrm{C}(13 \mathrm{~B})$ & $24(5)$ & $57(7)$ & $33(5)$ & $0(5)$ & $-18(4)$ & $-17(5)$ \\
$\mathrm{C}(14 \mathrm{~B})$ & $29(6)$ & $65(7)$ & $38(6)$ & $-7(5)$ & $5(5)$ & $-35(6)$ \\
$\mathrm{C}(15 \mathrm{~B})$ & $47(7)$ & $54(7)$ & $42(6)$ & $-1(5)$ & $-13(5)$ & $-33(6)$ \\
$\mathrm{C}(16 \mathrm{~B})$ & $38(6)$ & $30(6)$ & $43(6)$ & $-1(5)$ & $-7(5)$ & $-11(5)$ \\
$\mathrm{C}(17 \mathrm{~B})$ & $43(7)$ & $39(6)$ & $51(7)$ & $7(5)$ & $-6(6)$ & $-13(5)$ \\
& & & & & & \\
\hline
\end{tabular}


Table 5. Hydrogen coordinates ( x 10 $)$ and isotropic displacement parameters $\left(\AA^{2} \times 10^{3}\right)$ for d19133_a_5.

\begin{tabular}{|c|c|c|c|c|}
\hline & $\mathrm{x}$ & $\mathrm{y}$ & $\mathrm{z}$ & $\mathrm{U}(\mathrm{eq})$ \\
\hline $\mathrm{H}(1 \mathrm{AA})$ & 9200 & 1951 & 884 & 48 \\
\hline $\mathrm{H}(2 \mathrm{AA})$ & 11570 & 1350 & 22 & 51 \\
\hline $\mathrm{H}(3 \mathrm{AA})$ & 11508 & 1367 & -1225 & 57 \\
\hline $\mathrm{H}(4 \mathrm{AA})$ & 8874 & 2179 & -1577 & 49 \\
\hline $\mathrm{H}(7 \mathrm{AA})$ & 6237 & 3373 & -1792 & 42 \\
\hline $\mathrm{H}(8 \mathrm{AA})$ & 3439 & 4256 & -1895 & 46 \\
\hline $\mathrm{H}(9 \mathrm{AA})$ & 1442 & 4573 & -839 & 44 \\
\hline $\mathrm{H}(10 \mathrm{~A})$ & 2225 & 3924 & 310 & 42 \\
\hline $\mathrm{H}(13 \mathrm{~A})$ & 1202 & 958 & 1729 & 52 \\
\hline $\mathrm{H}(14 \mathrm{~A})$ & -829 & 3209 & 2231 & 55 \\
\hline $\mathrm{H}(15 \mathrm{~A})$ & -130 & 5177 & 2263 & 52 \\
\hline $\mathrm{H}(16 \mathrm{~A})$ & 2560 & 4912 & 1764 & 48 \\
\hline $\mathrm{H}(17 \mathrm{~A})$ & 5414 & 237 & 871 & 71 \\
\hline $\mathrm{H}(17 \mathrm{~B})$ & 4543 & -634 & 1437 & 71 \\
\hline $\mathrm{H}(17 \mathrm{C})$ & 3956 & -36 & 661 & 71 \\
\hline $\mathrm{H}(1 \mathrm{BA})$ & -1086 & 6785 & 4170 & 37 \\
\hline $\mathrm{H}(2 \mathrm{BA})$ & -2988 & 6342 & 5103 & 44 \\
\hline $\mathrm{H}(3 \mathrm{BA})$ & -3001 & 6667 & 6347 & 48 \\
\hline $\mathrm{H}(4 \mathrm{BA})$ & -1270 & 7620 & 6579 & 44 \\
\hline H(7BA) & 243 & 8802 & 6701 & 41 \\
\hline $\mathrm{H}(8 \mathrm{BA})$ & 2201 & 9680 & 6756 & 43 \\
\hline H(9BA) & 4143 & 9619 & 5695 & 44 \\
\hline $\mathrm{H}(10 \mathrm{~B})$ & 3966 & 8829 & 4598 & 38 \\
\hline $\mathrm{H}(13 \mathrm{~B})$ & 7795 & 5749 & 3350 & 43 \\
\hline $\mathrm{H}(14 \mathrm{~B})$ & 7809 & 7939 & 2793 & 48 \\
\hline $\mathrm{H}(15 \mathrm{~B})$ & 5254 & 9920 & 2627 & 51 \\
\hline $\mathrm{H}(16 \mathrm{~B})$ & 2790 & 9747 & 3117 & 46 \\
\hline H(17D) & 4479 & 5175 & 4357 & 71 \\
\hline $\mathrm{H}(17 \mathrm{E})$ & 6454 & 4403 & 4092 & 71 \\
\hline $\mathrm{H}(17 \mathrm{~F})$ & 5249 & 4437 & 3567 & 71 \\
\hline
\end{tabular}


Table 6. Torsion angles $\left[^{\circ}\right]$ for d19133_a_5.

\begin{tabular}{|c|c|}
\hline $\mathrm{C}(5 \mathrm{~A})-\mathrm{N}(1 \mathrm{~A})-\mathrm{C}(1 \mathrm{~A})-\mathrm{C}(2 \mathrm{~A})$ & $-1.4(15)$ \\
\hline $\mathrm{Ni}(1 \mathrm{~A})-\mathrm{N}(1 \mathrm{~A})-\mathrm{C}(1 \mathrm{~A})-\mathrm{C}(2 \mathrm{~A})$ & $169.0(8)$ \\
\hline $\mathrm{N}(1 \mathrm{~A})-\mathrm{C}(1 \mathrm{~A})-\mathrm{C}(2 \mathrm{~A})-\mathrm{C}(3 \mathrm{~A})$ & $-1.4(18)$ \\
\hline$C(1 \mathrm{~A})-\mathrm{C}(2 \mathrm{~A})-\mathrm{C}(3 \mathrm{~A})-\mathrm{C}(4 \mathrm{~A})$ & $2.2(17)$ \\
\hline$C(2 A)-C(3 A)-C(4 A)-C(5 A)$ & $-0.3(17)$ \\
\hline $\mathrm{C}(1 \mathrm{~A})-\mathrm{N}(1 \mathrm{~A})-\mathrm{C}(5 \mathrm{~A})-\mathrm{C}(4 \mathrm{~A})$ & $3.4(15)$ \\
\hline $\mathrm{Ni}(1 \mathrm{~A})-\mathrm{N}(1 \mathrm{~A})-\mathrm{C}(5 \mathrm{~A})-\mathrm{C}(4 \mathrm{~A})$ & $-168.3(8)$ \\
\hline$C(1 A)-N(1 A)-C(5 A)-C(6 A)$ & $-177.3(9)$ \\
\hline $\mathrm{Ni}(1 \mathrm{~A})-\mathrm{N}(1 \mathrm{~A})-\mathrm{C}(5 \mathrm{~A})-\mathrm{C}(6 \mathrm{~A})$ & $11.0(10)$ \\
\hline $\mathrm{C}(3 \mathrm{~A})-\mathrm{C}(4 \mathrm{~A})-\mathrm{C}(5 \mathrm{~A})-\mathrm{N}(1 \mathrm{~A})$ & $-2.6(16)$ \\
\hline$C(3 \mathrm{~A})-\mathrm{C}(4 \mathrm{~A})-\mathrm{C}(5 \mathrm{~A})-\mathrm{C}(6 \mathrm{~A})$ & $178.2(10)$ \\
\hline$C(10 A)-N(2 A)-C(6 A)-C(7 A)$ & $1.7(13)$ \\
\hline $\mathrm{Ni}(1 \mathrm{~A})-\mathrm{N}(2 \mathrm{~A})-\mathrm{C}(6 \mathrm{~A})-\mathrm{C}(7 \mathrm{~A})$ & $-179.8(7)$ \\
\hline$C(10 A)-N(2 A)-C(6 A)-C(5 A)$ & $179.4(8)$ \\
\hline $\mathrm{Ni}(1 \mathrm{~A})-\mathrm{N}(2 \mathrm{~A})-\mathrm{C}(6 \mathrm{~A})-\mathrm{C}(5 \mathrm{~A})$ & $-2.1(10)$ \\
\hline $\mathrm{N}(1 \mathrm{~A})-\mathrm{C}(5 \mathrm{~A})-\mathrm{C}(6 \mathrm{~A})-\mathrm{N}(2 \mathrm{~A})$ & $-6.0(12)$ \\
\hline $\mathrm{C}(4 \mathrm{~A})-\mathrm{C}(5 \mathrm{~A})-\mathrm{C}(6 \mathrm{~A})-\mathrm{N}(2 \mathrm{~A})$ & $173.3(10)$ \\
\hline$N(1 A)-C(5 A)-C(6 A)-C(7 A)$ & 171.7(9) \\
\hline$C(4 A)-C(5 A)-C(6 A)-C(7 A)$ & $-9.0(15)$ \\
\hline$N(2 A)-C(6 A)-C(7 A)-C(8 A)$ & $-1.7(14)$ \\
\hline$C(5 A)-C(6 A)-C(7 A)-C(8 A)$ & $-179.2(9)$ \\
\hline$C(6 A)-C(7 A)-C(8 A)-C(9 A)$ & $1.4(15)$ \\
\hline$C(7 A)-C(8 A)-C(9 A)-C(10 A)$ & $-1.1(16)$ \\
\hline $\mathrm{C}(6 \mathrm{~A})-\mathrm{N}(2 \mathrm{~A})-\mathrm{C}(10 \mathrm{~A})-\mathrm{C}(9 \mathrm{~A})$ & $-1.4(14)$ \\
\hline $\mathrm{Ni}(1 \mathrm{~A})-\mathrm{N}(2 \mathrm{~A})-\mathrm{C}(10 \mathrm{~A})-\mathrm{C}(9 \mathrm{~A})$ & $-179.7(8)$ \\
\hline $\mathrm{C}(8 \mathrm{~A})-\mathrm{C}(9 \mathrm{~A})-\mathrm{C}(10 \mathrm{~A})-\mathrm{N}(2 \mathrm{~A})$ & $1.2(16)$ \\
\hline$N(2 A)-N i(1 A)-C(11 A)-C(16 A)$ & $-90.4(9)$ \\
\hline $\mathrm{Br}(1 \mathrm{~A})-\mathrm{Ni}(1 \mathrm{~A})-\mathrm{C}(11 \mathrm{~A})-\mathrm{C}(16 \mathrm{~A})$ & $86.6(9)$ \\
\hline $\mathrm{N}(2 \mathrm{~A})-\mathrm{Ni}(1 \mathrm{~A})-\mathrm{C}(11 \mathrm{~A})-\mathrm{C}(12 \mathrm{~A})$ & $82.1(9)$ \\
\hline $\mathrm{Br}(1 \mathrm{~A})-\mathrm{Ni}(1 \mathrm{~A})-\mathrm{C}(11 \mathrm{~A})-\mathrm{C}(12 \mathrm{~A})$ & $-100.9(8)$ \\
\hline$C(16 A)-C(11 A)-C(12 A)-C(13 A)$ & $2.2(15)$ \\
\hline $\mathrm{Ni}(1 \mathrm{~A})-\mathrm{C}(11 \mathrm{~A})-\mathrm{C}(12 \mathrm{~A})-\mathrm{C}(13 \mathrm{~A})$ & $-170.5(7)$ \\
\hline$C(16 A)-C(11 A)-C(12 A)-C(17 A)$ & $-179.0(10)$ \\
\hline $\mathrm{Ni}(1 \mathrm{~A})-\mathrm{C}(11 \mathrm{~A})-\mathrm{C}(12 \mathrm{~A})-\mathrm{C}(17 \mathrm{~A})$ & $8.3(14)$ \\
\hline
\end{tabular}




\begin{tabular}{|c|c|}
\hline$C(11 A)-C(12 A)-C(13 A)-C(14 A)$ & $0.3(15)$ \\
\hline $\mathrm{C}(17 \mathrm{~A})-\mathrm{C}(12 \mathrm{~A})-\mathrm{C}(13 \mathrm{~A})-\mathrm{C}(14 \mathrm{~A})$ & $-178.6(10)$ \\
\hline $\mathrm{C}(12 \mathrm{~A})-\mathrm{C}(13 \mathrm{~A})-\mathrm{C}(14 \mathrm{~A})-\mathrm{C}(15 \mathrm{~A})$ & $-1.9(16)$ \\
\hline$C(13 A)-C(14 A)-C(15 A)-C(16 A)$ & $1.1(16)$ \\
\hline$C(14 A)-C(15 A)-C(16 A)-C(11 A)$ & $1.4(17)$ \\
\hline$C(12 A)-C(11 A)-C(16 A)-C(15 A)$ & $-3.0(16)$ \\
\hline $\mathrm{Ni}(1 \mathrm{~A})-\mathrm{C}(11 \mathrm{~A})-\mathrm{C}(16 \mathrm{~A})-\mathrm{C}(15 \mathrm{~A})$ & $169.5(9)$ \\
\hline $\mathrm{C}(5 \mathrm{~B})-\mathrm{N}(1 \mathrm{~B})-\mathrm{C}(1 \mathrm{~B})-\mathrm{C}(2 \mathrm{~B})$ & $-1.6(14)$ \\
\hline $\mathrm{Ni}(1 \mathrm{~B})-\mathrm{N}(1 \mathrm{~B})-\mathrm{C}(1 \mathrm{~B})-\mathrm{C}(2 \mathrm{~B})$ & $171.0(7)$ \\
\hline $\mathrm{N}(1 \mathrm{~B})-\mathrm{C}(1 \mathrm{~B})-\mathrm{C}(2 \mathrm{~B})-\mathrm{C}(3 \mathrm{~B})$ & $-1.0(15)$ \\
\hline $\mathrm{C}(1 \mathrm{~B})-\mathrm{C}(2 \mathrm{~B})-\mathrm{C}(3 \mathrm{~B})-\mathrm{C}(4 \mathrm{~B})$ & $3.0(16)$ \\
\hline $\mathrm{C}(2 \mathrm{~B})-\mathrm{C}(3 \mathrm{~B})-\mathrm{C}(4 \mathrm{~B})-\mathrm{C}(5 \mathrm{~B})$ & $-2.3(16)$ \\
\hline $\mathrm{C}(1 \mathrm{~B})-\mathrm{N}(1 \mathrm{~B})-\mathrm{C}(5 \mathrm{~B})-\mathrm{C}(4 \mathrm{~B})$ & $2.3(14)$ \\
\hline $\mathrm{Ni}(1 \mathrm{~B})-\mathrm{N}(1 \mathrm{~B})-\mathrm{C}(5 \mathrm{~B})-\mathrm{C}(4 \mathrm{~B})$ & $-171.4(8)$ \\
\hline$C(1 B)-N(1 B)-C(5 B)-C(6 B)$ & $-177.4(8)$ \\
\hline $\mathrm{Ni}(1 \mathrm{~B})-\mathrm{N}(1 \mathrm{~B})-\mathrm{C}(5 \mathrm{~B})-\mathrm{C}(6 \mathrm{~B})$ & $9.0(10)$ \\
\hline $\mathrm{C}(3 \mathrm{~B})-\mathrm{C}(4 \mathrm{~B})-\mathrm{C}(5 \mathrm{~B})-\mathrm{N}(1 \mathrm{~B})$ & $-0.3(16)$ \\
\hline $\mathrm{C}(3 \mathrm{~B})-\mathrm{C}(4 \mathrm{~B})-\mathrm{C}(5 \mathrm{~B})-\mathrm{C}(6 \mathrm{~B})$ & 179.4(10) \\
\hline $\mathrm{C}(10 \mathrm{~B})-\mathrm{N}(2 \mathrm{~B})-\mathrm{C}(6 \mathrm{~B})-\mathrm{C}(7 \mathrm{~B})$ & $-1.0(14)$ \\
\hline $\mathrm{Ni}(1 \mathrm{~B})-\mathrm{N}(2 \mathrm{~B})-\mathrm{C}(6 \mathrm{~B})-\mathrm{C}(7 \mathrm{~B})$ & $-177.2(7)$ \\
\hline $\mathrm{C}(10 \mathrm{~B})-\mathrm{N}(2 \mathrm{~B})-\mathrm{C}(6 \mathrm{~B})-\mathrm{C}(5 \mathrm{~B})$ & $-179.4(8)$ \\
\hline $\mathrm{Ni}(1 \mathrm{~B})-\mathrm{N}(2 \mathrm{~B})-\mathrm{C}(6 \mathrm{~B})-\mathrm{C}(5 \mathrm{~B})$ & $4.4(10)$ \\
\hline $\mathrm{N}(1 \mathrm{~B})-\mathrm{C}(5 \mathrm{~B})-\mathrm{C}(6 \mathrm{~B})-\mathrm{N}(2 \mathrm{~B})$ & $-8.9(12)$ \\
\hline $\mathrm{C}(4 \mathrm{~B})-\mathrm{C}(5 \mathrm{~B})-\mathrm{C}(6 \mathrm{~B})-\mathrm{N}(2 \mathrm{~B})$ & $171.5(10)$ \\
\hline$N(1 B)-C(5 B)-C(6 B)-C(7 B)$ & $172.8(9)$ \\
\hline $\mathrm{C}(4 \mathrm{~B})-\mathrm{C}(5 \mathrm{~B})-\mathrm{C}(6 \mathrm{~B})-\mathrm{C}(7 \mathrm{~B})$ & $-6.9(15)$ \\
\hline $\mathrm{N}(2 \mathrm{~B})-\mathrm{C}(6 \mathrm{~B})-\mathrm{C}(7 \mathrm{~B})-\mathrm{C}(8 \mathrm{~B})$ & $0.3(14)$ \\
\hline $\mathrm{C}(5 \mathrm{~B})-\mathrm{C}(6 \mathrm{~B})-\mathrm{C}(7 \mathrm{~B})-\mathrm{C}(8 \mathrm{~B})$ & $178.5(9)$ \\
\hline $\mathrm{C}(6 \mathrm{~B})-\mathrm{C}(7 \mathrm{~B})-\mathrm{C}(8 \mathrm{~B})-\mathrm{C}(9 \mathrm{~B})$ & $-0.7(15)$ \\
\hline $\mathrm{C}(7 \mathrm{~B})-\mathrm{C}(8 \mathrm{~B})-\mathrm{C}(9 \mathrm{~B})-\mathrm{C}(10 \mathrm{~B})$ & $1.8(16)$ \\
\hline $\mathrm{C}(6 \mathrm{~B})-\mathrm{N}(2 \mathrm{~B})-\mathrm{C}(10 \mathrm{~B})-\mathrm{C}(9 \mathrm{~B})$ & $2.2(15)$ \\
\hline $\mathrm{Ni}(1 \mathrm{~B})-\mathrm{N}(2 \mathrm{~B})-\mathrm{C}(10 \mathrm{~B})-\mathrm{C}(9 \mathrm{~B})$ & $177.9(8)$ \\
\hline $\mathrm{C}(8 \mathrm{~B})-\mathrm{C}(9 \mathrm{~B})-\mathrm{C}(10 \mathrm{~B})-\mathrm{N}(2 \mathrm{~B})$ & $-2.6(16)$ \\
\hline $\mathrm{N}(2 \mathrm{~B})-\mathrm{Ni}(1 \mathrm{~B})-\mathrm{C}(11 \mathrm{~B})-\mathrm{C}(16 \mathrm{~B})$ & $-86.9(9)$ \\
\hline $\mathrm{Br}(1 \mathrm{~B})-\mathrm{Ni}(1 \mathrm{~B})-\mathrm{C}(11 \mathrm{~B})-\mathrm{C}(16 \mathrm{~B})$ & $90.5(9)$ \\
\hline $\mathrm{N}(2 \mathrm{~B})-\mathrm{Ni}(1 \mathrm{~B})-\mathrm{C}(11 \mathrm{~B})-\mathrm{C}(12 \mathrm{~B})$ & $83.9(8)$ \\
\hline
\end{tabular}




$\begin{array}{lc}\mathrm{Br}(1 \mathrm{~B})-\mathrm{Ni}(1 \mathrm{~B})-\mathrm{C}(11 \mathrm{~B})-\mathrm{C}(12 \mathrm{~B}) & -98.8(8) \\ \mathrm{C}(16 \mathrm{~B})-\mathrm{C}(11 \mathrm{~B})-\mathrm{C}(12 \mathrm{~B})-\mathrm{C}(13 \mathrm{~B}) & 5.0(15) \\ \mathrm{Ni}(1 \mathrm{~B})-\mathrm{C}(11 \mathrm{~B})-\mathrm{C}(12 \mathrm{~B})-\mathrm{C}(13 \mathrm{~B}) & -166.1(7) \\ \mathrm{C}(16 \mathrm{~B})-\mathrm{C}(11 \mathrm{~B})-\mathrm{C}(12 \mathrm{~B})-\mathrm{C}(17 \mathrm{~B}) & -174.5(10) \\ \mathrm{Ni}(1 \mathrm{~B})-\mathrm{C}(11 \mathrm{~B})-\mathrm{C}(12 \mathrm{~B})-\mathrm{C}(17 \mathrm{~B}) & 14.4(14) \\ \mathrm{C}(11 \mathrm{~B})-\mathrm{C}(12 \mathrm{~B})-\mathrm{C}(13 \mathrm{~B})-\mathrm{C}(14 \mathrm{~B}) & -1.9(15) \\ \mathrm{C}(17 \mathrm{~B})-\mathrm{C}(12 \mathrm{~B})-\mathrm{C}(13 \mathrm{~B})-\mathrm{C}(14 \mathrm{~B}) & 177.7(10) \\ \mathrm{C}(12 \mathrm{~B})-\mathrm{C}(13 \mathrm{~B})-\mathrm{C}(14 \mathrm{~B})-\mathrm{C}(15 \mathrm{~B}) & -2.4(15) \\ \mathrm{C}(13 \mathrm{~B})-\mathrm{C}(14 \mathrm{~B})-\mathrm{C}(15 \mathrm{~B})-\mathrm{C}(16 \mathrm{~B}) & 3.5(15) \\ \mathrm{C}(14 \mathrm{~B})-\mathrm{C}(15 \mathrm{~B})-\mathrm{C}(16 \mathrm{~B})-\mathrm{C}(11 \mathrm{~B}) & -0.3(16) \\ \mathrm{C}(12 \mathrm{~B})-\mathrm{C}(11 \mathrm{~B})-\mathrm{C}(16 \mathrm{~B})-\mathrm{C}(15 \mathrm{~B}) & -4.0(16) \\ \mathrm{Ni}(1 \mathrm{~B})-\mathrm{C}(11 \mathrm{~B})-\mathrm{C}(16 \mathrm{~B})-\mathrm{C}(15 \mathrm{~B}) & 166.7(8)\end{array}$

Symmetry transformations used to generate equivalent atoms: 


\section{H. References}

${ }^{1}$ Meyer, G.; Troupel, M.; Périchon, J. J. Organomet. Chem. 1990, 393, 137-142.

${ }^{2}$ Harris, C. M.; McKenzie, E. D. J. Inorg. Nucl. Chem. 1967, 29, 1047-1068.

3 Yakhvarov, D.; Trofimova, E.; Sinyashin, O.; Kataeva, O.; Budnikova, Y.; Lönnecke, P.; HeyHawkins, E.; Petr, A.; Krupskaya, Y.; Kataev, V.; Klingeler, R.; Büchner, B. Inorg. Chem. 2011, 50, 4553-4558.

${ }^{4}$ Ni, S.; Padial, N. M.; Kingston, C.; Vantourout, J. C.; Schmitt, D. C.; Edwards, J. T.; Kruszyk, M. M.; Merchant, R. R.; Mykhailiuk, P. K.; Sanchez, B. B.; Yang, S.; Perry, M. A.; Gallego, G. M.; Mousseau, J. J.; Collins, M. R.; Cherney, R. J.; Lebed, P. S.; Chen, J. S.; Qin, T.; Baran, P. S. J. Am. Chem. Soc. 2019, 141, 6726-6739.

${ }^{5}$ Fieser, L. F.; Fieser, M. Fieser and Fieser's Reagents for Organic Synthesis, Volume 1; John Wiley and Sons, Inc.: New York, 1967.

${ }^{6}$ Mills, L. R.; Rousseaux, S. A. L. Tetrahedron 2019, 75, 4298-4306.

${ }^{7}$ Krasovskiy, A.; Malakhov, V.; Gavryushin, A.; Knochel, P. Angew. Chem. Int. Ed. 2006, 45, 6040-6044.

${ }^{8}$ Guilbault, G. G.; Kramer, D. N. Anal. Chem. 1965, 37, 1395-1399.

9 Jia, X.-G.; Guo, P.; Duan, J.; Shu, X.-Z. Chem. Sci. 2018, 9, 640-645.

${ }^{10} \mathrm{Yu}, \mathrm{P} . ;$ Morandi, B. Angew. Chem. Int. Ed. 2017, 56, 15693-15697.

${ }^{11}$ Shu, Z.; Ye, Y.; Deng, Y.; Zhang, Y.; Wang, J. Angew. Chem. Int. Ed. 2013, 52, 1057310576.

${ }^{12}$ Shigeno, M.; Hayashi, K.; Nozawa-Kumada, K.; Kondo, Y. Chem. Eur. J. 2019, 25, 60776081 .

${ }^{13}$ Wang, D. Y.; Yang, Z. K.; Wang, C.; Zhang, A.; Uchiyama, M. Angew. Chem. Int. Ed. 2018, 57, 3641-3645.

${ }^{14}$ Shen, T.; Wang, T.; Qin, C.; Jiao, N. Angew. Chem. Int. Ed. 2013, 52, 6677-6680.

15 Schedler, M.; Wang, D.-S.; Glorius, F. Angew. Chem. Int. Ed. 2013, 52, 2585-2589.

${ }^{16}$ McManus, J. B.; Nicewicz, D. A. J. Am. Chem. Soc. 2017, 139, 2880-2883.

17 Anbarasan, P.; Neumann, H.; Beller, M. Angew. Chem. Int. Ed. 2011, 50, 519-522..

18 Piontek, A.; Szostak, M. Eur. J. Org. Chem. 2017, 7271-7276.

${ }^{19}$ Reeves, J. T.; Malapit, C. A.; Buono, F. G.; Sidhu, K. P.; Marsini, M. A.; Sader, C. A.;

Fandrick, K. R.; Busacca, C. A.; Senanyake, C. H. J. Am. Chem. Soc. 2015, 137, 9481-9488.

${ }^{20}$ Stahl, M.; Korotkov, V. S.; Balogh, D.; Kick, L. M.; Gersch, M.; Pahl, A.; Kielkowski, P.;

Richter, K.; Schneider, S.; Sieber, S. A. Angew. Chem. Int. Ed. 2018, 57, 14602-14607.

${ }^{21}$ Grossman, O.; Gelman, D. Org. Lett. 2006, 8, 1189-1191.

22 Fang, W.-Y.; Qin, H.-L. J. Org. Chem. 2019, 84, 5803-5812.

${ }^{23}$ Miki, Y.; Hirano, K.; Tetsuya, M. M. Org. Lett. 2013, 15, 172-175.

${ }^{24}$ Cohen, D. T.; Buchwald, S. L. Org. Lett. 2015, 17, 202-205.

${ }^{25}$ Buitrago, E.; Tinnis, F.; Adolfsson, H. Adv. Synth. Catal. 2012, 354, 217-222.

${ }^{26}$ Zhang, X.; Xia, A.; Chen, H.; Liu, Y. Org. Lett. 2017, 19, 2118-2121.

27 Jhulki, S.; Seth, S.; Mondal, M.; Moorthy, J. N. Tetrahedron 2014, 70, 2286-2293.

${ }^{28}$ Iranpoor, N.; Panahi, F.; Roozbin, F.; Erfan, S.; Rahimi, S. Eur. J. Org. Chem. 2016, 17811787.

${ }^{29}$ Choy, P. Y.; Lau, C. P.; Kwong, F. Y. J. Org. Chem. 2011, 76, 80-84.

${ }^{30}$ Cho, J. Y.; Roh, B.; Cho, E. J. J. Org. Chem. 2018, 83, 805-811. 
${ }^{31}$ Shi, Y.-L.; Yuan, Q.; Chen, Z.-B.; Zhang, F.-L.; Liu, K.; Zhu, Y.-M. Synlett. 2018, 29, 359363.

${ }^{32}$ Moyano, E. L.; Colomer, J. P.; Yranzo, G. I. Eur. J. Org. Chem. 2008, 3377-3381.

${ }^{33}$ Liu, P.; Guo, J.; Wei, W.; Liu, X.; Sun, P. Eur. J. Org. Chem. 2016, 2105-2109.

${ }^{34}$ Senecal, T. D.; Shu, W.; Buchwald, S. L. Angew. Chem. Int. Ed. 2013, 52, 10035-10039.

35 Toma, G.; Fujita, K.-I.; Yamaguchi, R. Eur. J. Org. Chem. 2009, 4586-4588.

${ }^{36}$ Yang, L.; Liu, Y.-T.; Park, Y.; Park, S.-W.; Chang, S. ACS Catal. 2019, 4, 3360-3365.

${ }^{37}$ Collins, K. D.; Rühling, A.; Lied, F.; Glorius, F. Chem. Eur. J. 2014, 20, 3800-3805.

38 (a) Jiang, Y.; Wu, H.; Wu, H.; He, M. Synlett 2005, 2731-2734; (b) Puente, Á.; Ofial, A. R.; Mayr, H. Chem. Eur. J. 2017, 8, 1196-1202.

${ }^{39}$ Zysman-Colman, E.; Arias, K.; Siegel, J. S. Can. J. Chem. 2009, 87, 440-447.

40 Taherinia, Z.; Ghorbani-Choghamarani, A. Can. J. Chem. 2019, 97, 46-52.

${ }^{41}$ Zong, Y.; Hu, J.; Sun, P.; Jiang, X. Synlett. 2012, 23, 2393-2396.

${ }^{42}$ Mostafa, M. A. B.; Bowley, R. M.; Racys, D. T.; Henry, M. C.; Sutherland, A. J. Org. Chem. 2017, 82, 7529-7537.

${ }^{43}$ Huang, H.; Zong, H.; Bian, G.; Song, L. J. Org. Chem. 2015, 80, 12614-12619.

${ }^{44}$ Shrestha, T. B.; Troyer, D. L.; Bossmann, S. H. Synthesis 2014, 46, 646-652.

${ }^{45}$ Diness, F.; Fairlie, D. P. Angew. Chem. Int. Ed. 2012, 51, 8012-8016.

${ }^{46}$ Barraza, S. J.; Denmark, S. E. J. Am. Chem. Soc. 2018, 140, 6668-6684.

${ }^{47}$ Chen, S.; Yan, D.; Xue, M.; Hong, Y.; Yao, Y.; Shen, Q. Org. Lett. 2017, 19, 3382-3385.

${ }^{48}$ Liu, Z.; Zhang, J.; Chen, S.; Shi, E.; Xu, Y.; Wan, X. Angew. Chem. Int. Ed. 2012, 51, 32313235.

${ }^{49}$ Li, B.; Wendlandt, A. E.; Stahl, S. S. Org. Lett. 2019, 21, 1176-1181.

${ }^{50}$ Maetani, S.; Fukuyama, T.; Ryu, I. Org. Lett. 2013, 15, 2754-2757.

${ }^{51}$ Olsen, K. L.; Jensen, M. R.; MacKay, J. A. Tetrahedron Lett. 2017, 58, 4111-4114.

52 Ilangovan, A.; Satish, G. Org. Lett. 2013, 15, 5726-5729.

${ }^{53}$ Zhang, H.; Cai, Q.; Ma, D. J. Org. Chem. 2005, 70, 5164-5173.

${ }^{54}$ Keylor, M. H.; Niemeyer, Z. L.; Sigman, M. S.; Tan, K. L. J. Am. Chem. Soc. 2017, 139, $10613-10616$.

${ }^{55}$ Friis, S. D.; Pirnot, M. T.; Dupuis, L. N.; Buchwald, S. L. Angew. Chem. Int. Ed. 2017, 56, $7242-7246$.

56 Xong, X.; Tan, F.; Yeung, Y.-Y. Org. Lett. 2017, 19, 4243-4246.

57 Zhou, Y.; Zeng, Q.; Zhang, L. Synth. Comm. 2017, 47, 710-715.

${ }^{58}$ Leemhuis, M.; Van Steenis, J. H.; Van Uxem, M. J.; Van Nostrum, C. F.; Hennink, W. E. Eur. J. Org. Chem. 2003, 17, 3344-3349.

${ }_{59}$ Yao, Z.; Bhaumik, J.; Dhanalekshmi, S.; Ptaszek, M.; Rodriguez, P. A.; Lindsey, J. S.

Tetrahedron 2007, 63, 10657-10670.

${ }^{60}$ Andersen, V. L.; Herth, M. M.; Lehel, S.; Knudsen, G. M.; Kristensen, J. L. Tetrahedron Lett. 2013, 54, 213-216.

${ }^{61}$ Goossen, L. J.; Rodriguez, N.; Lange, P. P.; Linder, C. Angew. Chem. Int. Ed. 2010, 49, $1111-$ 1114.

${ }^{62}$ Weigel, W. K.; Dennis, T. N.; Kang, A. S.; Perry, J. J. P.; Martin, D. B. C. Org. Lett. 2018, 19, 6234-6238.

${ }^{63}$ He, Y.; Tang, J.; Luo, M.; Zeng, X. Org. Lett. 2018, 20, 4159-4163.

${ }^{64}$ Munday, R. H.; Martineli, J. R.; Buchwald, S. L. J. Am. Chem. Soc. 2008, 130, 2754-2755. 
${ }^{65}$ Lirag, R. C.; Miljanić, O. Š. Chem. Commun. 2014, 50, 9401-9404.

${ }^{66}$ Wang, Z.; Chang, S. Org. Lett. 2013, 15, 1990-1993.

${ }^{67}$ Pinxterhuis, E. B.; Visser, P.; Esser, I.; Gualtierotti, J.-B.; Feringa, B. L. Angew. Chem. Int. Ed. 2018, 57, 9452-9455.

${ }^{68}$ Nagano, T.; Hayashi, T. Org. Lett. 2005, 7, 491-493.

${ }^{69}$ Kaboudin, B.; Abedi, Y.; Yokomatsu, T. Eur. J. Org. Chem. 2011, 33, 6656-6662. 